\title{
Conformational Equilibria of Organic Adsorbates on Nanostructures in Aqueous Solution. MD Simulations
}

\section{Dissertation}

zur Erlangung des akademischen Grades eines Doktors der

Naturwissenschaften

- Dr. rer. nat. -

vorgelegt von

Amal Kanta Giri

geboren in Paikbarh, West Bengal, India

\section{UNIVERSITÄT \\ $D_{E} U_{S} I_{S} S_{N} U R$ G}

Offen im Denken

\author{
Fakultät für Chemie \\ der Universität Duisburg-Essen
}

2016 

Die vorliegende Arbeit wurde im Zeitraum von September 2012 bis April 2016 im Arbeitskreis von Prof. Dr. Eckhard Spohr im Institut für Theoretische Chemie der Universität Duisburg-Essen durchgeführt.

Tag der Disputation: 30/06/16

Gutachter: $\quad$ Prof. Dr. Eckhard Spohr Prof. Dr. Georg Jansen

Vorsitzender: Prof. Dr. Karin Stachelscheid 



\section{Acknowledgements}

I would like to express my sincere gratitude to my advisor Prof. Eckhard Spohr, who gave me opportunity to pursue my doctoral research in his supervision. His versatile knowledge helped me to enrich my expertise and broaden my views. I would like to thank him for his guidance, novel ideas and advices, which helped me to do my research work smoothly. I sincerely thank to Prof. Georg Jansen for his comments and suggestions, and also, I appreciate him for many memorable conversations.

I appreciate PD. Dr. Holger Somnitz for valuable discussions and I would like to thank him for the review of abstract of this thesis. I specially thank to Dr. Volodymyr P. Sergiievskyi and Dr. Jure Gujt for the critical reviews of this thesis. Many thanks to Michael Marcinkowski for his help to translate the abstract from English to German. I wish to thank Dr. Liane Lorbeer, Dr. Johannes Wiebe and Ramona Ufer for their helps, which made my life easy in Germany. I would like to thank my colleagues and members of theoretical chemistry at University of Duisburg-Essen for their cooperation. Finally, I thank my parents and friends for supporting me throughout all the difficulties. 

To my parents...... 



\section{Abstract}

Atomistic molecular dynamics (MD) simulations of bulk aqueous solution of $\mathrm{NaCl}$ and functionalized gold nanoparticles (GNPs) in aqueous solution were conducted at $300 \mathrm{~K}$. Bulk $\mathrm{NaCl}$ solution systems of different concentration have been studied using various force field parameter sets to investigate aggregation of ions and to choose suitable force field parameters for ions. Gold nanoparticles functionalized with linear and branched hydrocarbon chains with different grafting density $\left(d_{f}=1,2 / 3\right.$ and $\left.1 / 3\right)$, chain length $\left(\left(\mathrm{CH}_{2}\right)_{l}, l=5,11,17\right.$ and 23), position of the branching point (4th, 8th and 12th carbon) and terminal groups $\left(\mathrm{CH}_{3}, \mathrm{COO}^{-}\right.$and $\left.\mathrm{NH}_{3}^{+}\right)$were modeled to investigate various phenomena at the microscopic level. The solution of a functionalized GNP with ionic terminations was neutralized by excess $\mathrm{Na}^{+}$and $\mathrm{Cl}^{-}$ions. Focus has been given to the penetration depth of water and ions into the diffuse shell of alkanethiols as a function of grafting density, functionalization and chain length. Special focus has been given to the relative accessibility of the gold core by water in dependence of grafting density, chain length and chain shape. The orientation of water molecules inside the hydrocarbon chain environment (from the surface of the gold core to the terminal groups) and near the terminal groups has been studied in detail. Hydrogen bonds between water and polar terminal groups have also been studied. Special attention has been given to the solvent accessible surface area (SASA) of functionalized GNPs, and the coating asymmetry and ways to prevent it.

It is observed that the penetration of water and ions into the hydration shell of alkanethiols increases with decreasing grafting density, irrespective of the terminal group of the hydrocarbon chains. High grafting densities lead to more extended hydrocarbon chains which behave more rigidly. The solvent accessible surface area (SASA) is dependent on the chain length and the terminal group. SASA (per unit chain length) of $\mathrm{COO}^{-}$group terminated GNPs with chain length $\left(C_{l}\right) 5$ is the highest among all GNP coated with linear chains. Water molecules orient near the surface of the gold core pointing one hydrogen 
towards the center of the gold core irrespective of chain length, terminal group and grafting density. Water molecules cannot access the surface of the gold core of a branched chain GNP as easily as in the linear chain GNP. Branched chains protect the core better than the corresponding linear chains. Among all studied systems, nonpolar group terminated branched chains with chain length 17 and branched at the 4th carbon atom provide the best protection to the nanoparticle core. Coating asymmetry is generally more prominent for longer chains $(>11)$, and the asymmetry can be prevented by introducing branching points. 


\section{Zusammenfassung}

Es wurden Molekulardynamik-Simulationen (MD) zum einen von $\mathrm{NaCl}$ in Wasser und zum anderen von funktionaliserten Gold-Nanopartikeln in Wasser bei einer Temperatur von $300 \mathrm{~K}$ durchgeführt. Hierbei wurden variable $\mathrm{NaCl}$ Konzentrationen mit jeweils verschiedenen Kraftfeldern untersucht, um die Aggregation von Ionen zu beobachten und geeignete Kraftfeldparameter für Ionen zu identifizieren. Funktionalisierte Nanopartikel mit linearen und verzweigten Kohlenwasserstoffen unterschiedlicher Pfropfdichte $\left(d_{f}=1\right.$, $2 / 3$ und $1 / 3)$, Kettenlänge $\left(\left(\mathrm{CH}_{2}\right)_{l}, l=5,11,17\right.$ und 23), Kettenart (linear oder verzweigt), Position der Verzweigung (4., 8. und 12.) sowie Art der Endgruppe $\left(\mathrm{CH}_{3}, \mathrm{COO}^{-}\right.$und $\mathrm{NH}_{3}^{+}$) wurden modelliert, um diverse Phänomene auf mikroskopischer Ebene zu untersuchen. Durch einen Überschuss von $\mathrm{Na}^{+}$und $\mathrm{Cl}^{-}$Ionen wurden die ionischen Reste der funktionalisierten Gold-Nanopartikel neutralisiert. Der Fokus wurde auf die Eindringtiefe des Wassers sowie der Ionen in die diffuse Hülle der Alkanthiole in Abhängigkeit der Pfropfdichte, Funktionalisierung und Kettenlänge gelegt. Des Weiteren wurde die Zugänglichkeit und relative Verfügbarkeit der Goldoberfläche für Wasser in Abhängigkeit der Propfdichte, Funktionalisierung und Kettenlänge studiert. Die Orientierung der Wassermoleküle innerhalb der Kohlenwasserstoffumgebung (von der Oberfläche des Goldkerns bis hin zu der terminalen Gruppe) und nahe der Endgruppen wurde dabei detailliert untersucht. Ebenfalls analysiert wurden die Wasserstoffbrücken zwischen Wasser und den polaren Endgruppen. Besondere Aufmerksamkeit wurde auf die dem Lösungsmittel zugängliche Oberfläche (engl. SASA) und der Beschichtungsasymmetrie der funktionaliserten Gold-Nanopartikel gelegt sowie darauf, wie eine solche Asymmetrie verhindert werden kann.

Er wird beobachtet, dass die Eindringtiefe von Wasser und Ionen in die Hydratationshülle der Alkanthiole mit abnehmender Pfropfdichte zunimmt, und zwar unabhängig von der Art der Endgruppe. Hohe Pfropfdichten führen zu verlängerten Kohlenwasserstoffketten, die sich steif verhalten. Die dem Lösungsmittel zugängliche Oberfläche (engl. 
SASA) ist sowohl von der Kettenlänge als auch von der Endgruppe abhängig. Diese ist im Fall der $\mathrm{COO}^{-}$terminierten Ketten mit der Kettenlänge $\left(C_{l}\right)$ von 5 die höchste unter allen Gold-Nanopartikeln mit linearen Ketten. Die Wassermoleküle orientieren sich nahe der Oberfläche des Goldkerns, unabhängig von der Kettenlänge, Endgruppe und Pfropfdichte, so dass je ein H-Atom eines Wassermoleküls sich zur Mitte des Goldkerns hin ausrichtet. Die Wassermoleküle können die Oberfläche der Gold-Nanopartikel bei verzweigten Kohlenwasserstoffen nicht so einfach erreichen wie es bei linearen Ketten der Fall ist. Daraus folgt, dass verzweigte Ketten den Kern deutlich besser schützen als vergleichbare lineare Ketten. Unter allen untersuchten Systemen bieten die am 4. Kohlenwasserstoffatom mit unpolaren Rest verzweigten Ketten mit der Kettenlänge 17 den besten Schutz für den Kern des Nanopartikels. Beschichtungsasymmetrie wird für längere Ketten (>11) gefunden, und die Aymmetrie kann verhindert werden, wenn Verzweigugnspunkte eingeführt werden. 


\section{Glossary}

$\begin{aligned} \text { AA } & \text { All atom } \\ \text { acf } & \text { Autocorrelation function } \\ \text { AMBER } & \text { Assisted model building with energy refinement } \\ \text { CHARMM } & \text { Chemistry at Harvard macromolecular mechanics } \\ \text { CG } & \text { Coarse grain } \\ C_{l} & \text { Chain length } \\ d_{f} & \text { Degree of functionalization } \\ \text { DFT } & \text { Density functional theory } \\ \text { FMNP } & \text { Functionalized metal nanoparticle } \\ \text { GNP } & \text { Gold nanoparticle } \\ \text { GNPS } & \text { Gold nanoparticles } \\ \text { K } & \text { Kelvin } \\ \text { LAMMPS } & \text { Large-scale atomic or molecular massively parallel simulator } \\ \text { LCFGNP } & \text { Linear chain functionalized gold nanoparticle } \\ \mathbf{m} & \text { molal } \\ \mathbf{M D} & \text { Molecular dynamics } \\ \mathbf{n m} & \text { Nanometer } \\ \mathbf{N P} & \text { Nanoparticle }\end{aligned}$


NPT Number of particle, pressure and temperature are constant

NPs Nanoparticles

NVT Number of particle, volume and temperature are constant

ns Nanosecond

OPLS Optimized potentials for liquid simulations

PPPM Particle-particle particle-mesh

$R_{g} \quad$ Radius of gyration

SASA Solvent accessible surface area

SDCs Self diffusion coefficients

TAAD Time averaged angle distribution

TIP3P Three-site transferable intermolecular potential

VMD Visual molecular dynamics

VOSS Volume occupied by solute in solution 


\section{Contents}

Glossary $\quad$ xi

1 Introduction $\quad 1$

2 Theoretical Overview $\quad 5$

2.1 Molecular Dynamics Simulation . . . . . . . . . . . . . . 6

2.1.1 Verlet Algorithm . . . . . . . . . . . . . . . . 7

2.2 Force Field Models . . . . . . . . . . . . . . . . . . . . . . . . 9

2.2.1 Bonded Interactions . . . . . . . . . . . . . . . 11

2.2.2 Nonbonded Interactions . . . . . . . . . . . . . . . . . . . 13

2.3 Thermostating and Barostating . . . . . . . . . . . . . 15

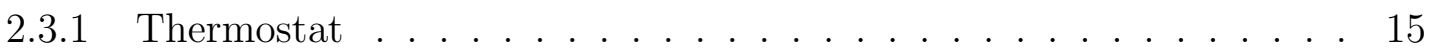

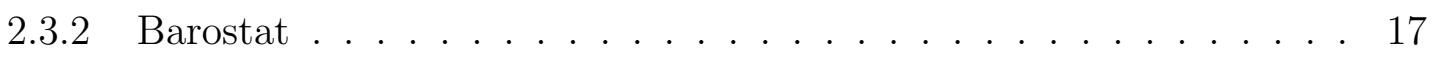

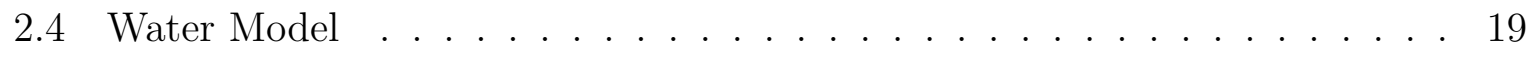

2.5 Analysis Tools . . . . . . . . . . . . . . . . . . . . 20

2.5.1 Radial Distribution Function . . . . . . . . . . . . . 20

2.5.2 Center of Mass . . . . . . . . . . . . . . . . 21

2.5.3 Radius of Gyration . . . . . . . . . . . . . . . 22

2.5.4 Diffusion Coefficient . . . . . . . . . . . . . . . . 23 
2.5.5 Hydrogen Bonds . . . . . . . . . . . . . . . . . . . 24

3 Cluster Formation of $\mathrm{NaCl}$ in Bulk Solutions $\quad 27$

3.1 Simulation Details . . . . . . . . . . . . . . . . 27

3.2 Results and Discussion . . . . . . . . . . . . . . . . 28

4 Linear Chain Functionalized Gold Nanoparticle in Aqueous Solution 35

4.1 Modeling and Method ... . . . . . . . . . . . . 35

4.2 Results . . . . . . . . . . . . . . . . . . . . 40

4.2 .1 Shape of Gold Nanoparticles . . . . . . . . . . . . . . . . . 40

4.2 .2 Penetration of Water and Ions . . . . . . . . . . . . . 42

4.2.3 Conformation of Hydrocarbon Chains . . . . . . . . . . . . . . 48

4.2.4 Mobility of Ions and Hydrogen bonds . . . . . . . . . . . . . 51

4.2.5 Integrated Charge and Water Orientation . . . . . . . . . 54

5 Coating Asymmetry on Gold Cluster and its Prevention 65

5.1 System Preparation and Methods . . . . . . . . . . . . . . 65

5.2 Results and Discussion . . . . . . . . . . . . . . . . . 71

5.2.1 Chain Length Dependence of Coating Asymmetry . . . . . . . . . 71

5.2.2 Branched Chains and Prevention of Coating Asymmetry . . . . . 75

5.2 .3 Surface Access of GNPs . . . . . . . . . . . . . . . 80

5.2.4 Water Penetration and Shape of GNPs . . . . . . . . . . 85

5.2.5 Solvent Accessible Surface Area . . . . . . . . . . . . . . . 87

$\begin{array}{llr}6 & \text { Conclusions } & 93\end{array}$

$\begin{array}{lr}\text { List of Figures } & 98\end{array}$ 
List of Tables

102

References

115

Appendices

117

A.1 Locations of files . . . . . . . . . . . . . . . . . . 117

A.2 Color maps of surface access of $\mathrm{NH}_{3}^{+}$terminated systems with different grafting densities . . . . . . . . . . . . . . . 120

A.3 Time averaged angle distribution . . . . . . . . . . . . . . . . . 121

A.4 Color maps of surface access with different chain length . . . . . . . . . . 122

A.5 Color maps of surface access of branched chain systems of chain length 11. 123

A.6 Radius of gyration and time averaged chain length of $\mathrm{NH}_{3}^{+}$group terminated chains . . . . . . . . . . . . . . . . . . . . 124 


\section{Introduction}

Nanoparticles with diameters of 1-100 nm have recently become a focus of material research, since they can be used in a variety of applications such as electronics, ${ }^{1-3}$ optics, ${ }^{4,5}$ chemistry, ${ }^{6,7}$ biosensing, ${ }^{8-11}$ imaging, ${ }^{12-14}$ drug delivery, ${ }^{15-18}$ catalysis ${ }^{19,20}$ and micro fabrication. ${ }^{21,22}$ Such nanoparticles bridge the gap between the bulk material and the atomic or molecular structure. ${ }^{23,24}$ Normally, the physical properties of bulk material do not change with the size of the material, but the properties of nanoparticles change with the size of the particle. For small nanoparticles of size less than $10 \mathrm{~nm}$, electronic structure plays an important role to determine stability, composition and structure of the particle. ${ }^{25,26}$ Aggregation of nanoparticles is a matter of concern for bulk handling of nanoparticle based materials. ${ }^{27}$ Short hydrocarbon chain coated nanoparticles can be a means to prevent aggregation of the naked particles. There are many properties of hydrocarbon chain coated nanoparticles or functionalized nanoparticles which help to form complex superstructures. Functionalized nanoparticles are often made of long hydrocarbon chains with terminal functional groups and metal core. The long hydrocarbon chains are chemically bonded to the metal core via different groups. In many cases, for gold nanoparticles in particular, the chains are bonded via sulfur bridges. These functionalized metal nanoparticles (FMNPs) ${ }^{28-33}$ can be tuned easily by changing the size and shape of metal core, chain length and shape, and number of chains on the metal surface. Different properties like aggregation, electrochemistry and solubility can be changed very easily by modifying the terminal groups of the hydrocarbon chains ranging from more hydrophilic, charged or neutral groups $\left(\mathrm{COO}^{-}, \mathrm{OH}, \mathrm{NH}_{3}^{+}\right)$to hydrophobic $\left(\mathrm{CH}_{3}, \mathrm{CF}_{3}\right)$ ones.

Various metals like gold, silver and platinum can form the core of functionalized nanoparticles. One reason to use metals to form the core is that metals have large surface area to volume ratio which enables to add hundreds of polymers on the surface of

metal core. However, gold is widely used to form the core due its unique properties and 
non-toxicity. Also, gold does not form stable oxides, thus it can be handled easily. Gold nanoparticles have attractive optical properties, and the color of nanoparticle solutions can vary between yellow and dark green, depending on the particle size. Also, its light scattering properties are very interesting, and these properties are used in biological imaging applications. ${ }^{34}$ If gold nanoparticles (GNPs) are excited by light of wavelength from 700-800 nm, they produce heat, and this property can be used to kill cancer cells inside the human body. Also, absorption of sunlight by semiconductor materials made of nanoparticles is much higher than by a continuous sheet of a thin film. GNPs are more stable than other metallic NPs in aqueous solution. In other work it has been shown that the grafting density of chains on a NP plays an important role to form composite materials. ${ }^{35}$ Additionally, the hydrocarbon chain length and the terminal groups change properties such as solubility, dispersion and aggregation of the functionalized gold nanoparticle in solution, and these properties play a vital role to form complex super structures or to handle nanoparticle based bulk materials. So the fundamental study of different properties of functionalized gold nanoparticle at the microscopic level is essential.

To gain knowledge on stabilisation, it is necessary to understand the role of functionalized GNPs in biological environment or in crowded solution of ions. Compared to a experimental study, computer simulations offer detail information about interactions between functionalized GNP and aqueous solution at the atomic level. Few studies of such particles in aqueous or biological environment have been performed to date and most of them are MD simulations. Ghorai and Glotzer ${ }^{36}$ performed atomistic MD simulations of self-assembled alkanethiol monolayers on NPs in vacuum to investigate the ligand shell organization of homoligand surfactants on spherical GNP surfaces as a function of temperature. They compared the structure of monolayers on GNP surface with the structure on flat gold surface. They found that at room temperature or slightly elevated temperature chains on flat surfaces tilt mostly in one particular direction but on spherical surfaces a group of chains tilt together in various directions due to curvature induced disclinations. They also found that at high temperature, chains on spherical surface orient randomly. Luedtke and Landman ${ }^{37}$ studied dodecanethiol self-assembled monolayers on small and large gold nanocrystallites. They investigated the equilibrium structure and thermodynamic properties of the particles in butanethiol. They showed the temperature dependence of bundle formation and conformational defects of chains.

In a pioneering study, the group of Grest ${ }^{38,27}$ investigated coating structure and solubility of functionalized gold nanoparticles in water and decane. They also studied 
effects of ions on coating asymmetry, and orientation of coatings near the water-vapor interface. Yang and Chen ${ }^{39}$ investigated structure and dynamic properties, hydrogen bond dynamics and far-infrared (far-IR) spectra of hydration water molecules around the mixed monolayer-protected gold nanoparticles with different ligand compositions and length. Yang and Weng ${ }^{40}$ studied the structure and dynamics of pure water around a polar and nonpolar functionalized GNP with methyl, carboxyl, amine and hydroxyl termination in much detail employing MD. Cruz and coworkers ${ }^{41}$ studied surface patterns of charged end group ligand attached nanoparticles and various conformations of the ligand coatings. Heikkilä and coworkers ${ }^{42}$ studied charged monolayer-protected GNPs in dilute solutions. They investigated complexes formed between charged GNPs, and water and ions around it. Also they investigated interactions between charged GNPs and lipid bilayers. ${ }^{43}$ Alfredo and coworkers ${ }^{44}$ investigated aggregation of two small, charged, alkanethiol monolayer-protected gold nanoparticles under typical biological conditions using implicit solvent simulations. Furthermore, they investigated the role of ligand-ligand interaction for the stabilisation of the particles. Zhang and coworkers ${ }^{45}$ studied aggregation of monolayer-protected GNPs using a coarse-grained (CG) molecular dynamics approach. They investigated the dependence of aggregation on the chain length and terminal groups, and the effect of salt concentration on aggregation. Recently, the Grest group calculated potentials of mean force (PMFs) between nanoparticles vs coating thickness. ${ }^{46}$ They found that the force at separations beyond about 2.5 nanoparticle radii is statistically very insignificant, but becomes quickly repulsive for distances smaller than about 2 nanoparticle radii. In other simulation work different aspects have been investigated such as bundle formation and stability, ${ }^{47-50}$ interaction between the particles, ${ }^{51-53}$ dynamics of the particle ${ }^{50,54}$ chain structure ${ }^{54}$ and solubility. ${ }^{49}$ Some of these studies have been performed using MD with all atom or united atom force fields; other studies have been performed employing coarse-grained methods. Also, simulation of functionalized gold nanoparticles in supercritical $\mathrm{CO}_{2}$ has been reported. ${ }^{55,56}$

In this $\mathrm{PhD}$ thesis, charged and uncharged, partially or fully functionalized GNPs in an approximately 1 molar $\mathrm{NaCl}$ solution have been studied. The core of the GNPs is around $2 \mathrm{~nm}$ in diameter, and it has three layers which consist of 144 gold atoms. The core was modeled in the same way as a DFT model taken from the literature. ${ }^{57}$ The core is functionalized with $-\left(\mathrm{CH}_{2}\right)_{x}-\mathrm{CH}_{3},-\left(\mathrm{CH}_{2}\right)_{x}-\mathrm{COO}^{-}$and $-\left(\mathrm{CH}_{2}\right)_{x}-\mathrm{NH}_{3}^{+}$chains and branched chains, and the chains are attached to the gold core via sulfur bridges. Specifically, different systems have been studied varying the chain length (from 5 to 23 
atoms), terminal groups $\left(\mathrm{CH}_{3}, \mathrm{COO}^{-}\right.$and $\left.\mathrm{NH}_{3}^{+}\right)$and branching position (4th, 8th and 12 th carbon atom or none at all). One goal was to elucidate the relative accessibility of the particle surface by water in the presence of the grafted hydrocarbon chains of varying termination, grafting density, chain length, and branching position. Focus has been given on the water density and orientation, around the NP from the gold surface up to the terminal groups. Hydrogen bonds between polar terminals and water molecules, ion contact, and integrated charge density from the surface to the terminal group have been studied. Attention also has been given to prevention of coating asymmetry and solvent accessible surface area (SASA). 


\section{Theoretical Overview}

Science needs both observation and comprehension. Without observation there are no facts to be comprehended; without comprehension science is mere documentation. ${ }^{58}$ The basis of comprehension of an observation is theory. Usually, there is always a chasm between the statement of a theory and the ability to extract information to interpreting experiment. ${ }^{58}$ In reality, exact solutions of different problems are notable exceptions. Therefore, theory relies on approximations, often this approximation is uncontrolled, and so the reliability is questionable. In this regard, simulation is better than theory. Simulation rests on basic theoretical foundations, but avoids much of the approximations associated with theory, by replacing the approximations with more elaborate calculations. ${ }^{58}$

Some problems in statistical mechanics are exactly soluble. This means that a complete specification of the microscopic properties of a system leads directly and easily to a set of interesting results or macroscopic properties. In statistical mechanics, there are few non-trivial problems which are exactly solvable, like the ideal gas, the harmonic crystal, and the two-dimensional Ising model for ferromagnets. Some problems in statistical mechanics are not solvable exactly, deploying approximations in analytical methods. ${ }^{59}$ Therefore, to solve such problems, computer simulation play a pivotal role.

When the computer is used for simulation, the computer becomes a "virtual laboratory", in which a system is studied. ${ }^{58}$ Simulation plays a dual role: on one hand it bridges between model and prediction from theory, and on the other hand it bridges between model and experiment. The simulation provides in principle a direct route from microscopic details of a system to macroscopic properties. The results of computer simulations can be compared with real experiment. The results emerging from a computer simulation may differ strongly from experiment, and this largely depends on the tech-

nique used in simulation and on the quality of the interaction model. ${ }^{58} \mathrm{~A}$ wide variety 
of simulation techniques has been developed over the years such as: Molecular Dynamics, Classical Monte Carlo, quantum based techniques involving path integral and Monte Carlo methods, and MD combined with quantum mechanical density functional theory. If the simulation technique is appropriate, then it can unveil the inside story and can help experimentalists to interpret new results.

Furthermore, it may be difficult to perform experiments under extreme pressure and temperature, but computer simulations are possible without any difficulties. Details of molecular structure and motion are difficult to probe experimentally, but can be extracted easily from a simulation. The scope of computer simulation is not limited to solving some non-trivial statistical mechanics problems, rather it extends to investigate more complex systems such as DNA, liquid crystals, polymers, lipid membranes and viruses. In the field of liquid matter and biology, nowadays, simulation often comprises fully hydrated systems with hundred of thousands of atoms and extended over hundreds of nanoseconds. ${ }^{58,60,61}$

\subsection{Molecular Dynamics Simulation}

Classical molecular dynamics (MD) is one of the simulation techniques used for computing equilibrium and transport properties of a classical many body systems. This technique is widely used for large-scale simulations at the molecular level in different fields. In MD successive configurations of the systems are generated by integrating Newton's equations of motion. The successive configurations, or the "trajectories", specify how the positions and velocities of particles in the system vary with time. Newton's second law of motion says: force on a particle is equal to the rate of change of its momentum $\left(\vec{F}=\frac{d \vec{p}}{d t}\right)$. The trajectory is obtained by solving the equation embodied in Newton's second law $\left(\vec{F}=\frac{d \vec{p}}{d t}\right):{ }^{62,63}$

$$
\frac{d^{2} x_{i}}{d t^{2}}=\frac{F_{x_{i}}}{m_{i}}
$$

This equation describes the motion of a particle of mass $\mathrm{m}_{i}$ along $\mathrm{x}_{i}$ direction when a force $\mathrm{F}_{x_{i}}$ acts on the particle in the same direction.

A simple molecular dynamics program follows the following steps: ${ }^{62}$

1. At first, it reads the initial configuration such as positions and velocities of particles inside a system, and conditions (e.g. initial temperature, density, time steps, and 
different constraints).

2. Then, it computes forces on all particles.

3. After that, integrating Newton's equation of motion, it updates positions and velocity of particles using a small time step $\delta t$. This process is repeated for the desired number of time steps.

4. Then, it prints the calculated quantities and stops.

\subsubsection{Verlet Algorithm}

The most widely used method to solve Newton's equation of motion (2.1) and to calculate the trajectory of particles in MD simulation is the Verlet algorithm. The main idea behind this method is that the integration is broken down into many small steps, each separated by small fixed time $\delta t$. The total force on each particle in the simulated system at a time $t$ is calculated as the vector sum of its interactions with other particles in the system. From the force on particles, their acceleration can be calculated. Combining the acceleration with the position and velocity of the particles at a time $t$, position and velocity of particles can be calculated at a time $t+\delta t$. The forces on the particles are assumed to be constant during the small time step $\delta t$. The forces on the particles in their new positions are then calculated at time $t+\delta t$ to get positions and velocities at time $t+2 \delta t$. This process runs iteratively up to a desired time length to get a trajectory. Mathematically, the Verlet algorithm can be represented as follows:

Position and acceleration of particles at time $t$ and position of particles from the previous step $t-\delta t$ are used to calculate new positions at time $t+\delta t$. Taylor expansions of the position of a particle at times $t+\delta t$ and $t-\delta t$ are a $^{59,62}$

$$
\begin{aligned}
& \vec{r}(t+\delta t)=\vec{r}(t)+\delta t \vec{v}(t)+\frac{1}{2} \delta t^{2} \vec{a}(t)+\frac{\delta t^{3}}{3 !} \dot{\vec{a}}+\mathcal{O}\left(\delta t^{4}\right) \\
& \vec{r}(t-\delta t)=\vec{r}(t)-\delta t \vec{v}(t)+\frac{1}{2} \delta t^{2} \vec{a}(t)-\frac{\delta t^{3}}{3 !} \dot{\vec{a}}+\mathcal{O}\left(\delta t^{4}\right)
\end{aligned}
$$

In these equations $\vec{r}, \vec{v}, \vec{a}, \dot{\vec{a}}$, and $\mathcal{O}$ are position, velocity, acceleration, time derivative of acceleration and order of error, respectively. Summing the equations 2.2 and 2.3 gives ${ }^{59}$

$$
\vec{r}(t+\delta t) \approx 2 \vec{r}(t)-\vec{r}(t-\delta t)+\delta t^{2} \vec{a}(t)
$$


Equation 2.4 does not use velocity to calculate the new position at time $t+\delta t$. The velocity can be calculated in many different ways. A simple way is to use the knowledge of the trajectory ${ }^{59}$

$$
\vec{r}(t+\delta t)-\vec{r}(t-\delta t)=2 \vec{v}(t) \delta t+\mathcal{O}\left(\delta t^{3}\right)
$$

or

$$
\vec{v}(t)=\frac{\vec{r}(t+\delta t)-\vec{r}(t-\delta t)}{2 \delta t}+\mathcal{O}\left(\delta t^{2}\right)
$$

Here the velocity is accurate to the order of $\delta t^{2}$, but more accurate estimate of the velocity is possible using a Verlet-like algorithm. The Verlet algorithm is simple and straightforward. It stores two sets of positions and acceleration to calculate new positions of particles. It is economic in its use of storage. One of the disadvantages of the algorithm is that the new position $\vec{r}(t+\delta t)$ at time $t+\delta t$ is obtained by adding a small term $\delta t^{2} \vec{a}(t)$ to the difference between two big terms of $\vec{r}(t)$ and $\vec{r}(t-\delta t)$ (see equation 2.4). This disadvantage may lead to the loss of precession. Another drawback is that the velocity term is not present explicitly in the equations and velocity is not available until the position have been calculated at the next step. One further drawback is that the new position $\vec{r}(t+\delta t)$ depends on the current position $\vec{r}(t)$ and the position form the previous time step $t-\delta t$. So, at time $t=0$ there is only one set of positions, another set of positions at $t=-\delta t$ has to be provided by other means.

Several variations of Verlet algorithm have been developed. MD software package LAMMPS uses the velocity Verlet method which yields positions, velocities and accelerations at the same instant of time without compromising precision. ${ }^{59}$

$$
\begin{gathered}
\vec{r}(t+\delta t) \approx \vec{r}(t)+\delta t \vec{v}(t)+\frac{1}{2} \delta t^{2} \vec{a}(t) \\
\vec{v}(t+\delta t) \approx \vec{v}(t)+\delta t \vec{a}(t)+\frac{1}{2} \delta t^{2} \dot{\vec{a}}(t) \\
\vec{a}(t+\delta t) \approx \vec{a}(t)+\delta t \dot{\vec{a}}(t)
\end{gathered}
$$

From equation 2.7 and 2.8

$$
\vec{v}(t+\delta t)=\vec{v}(t)+\frac{1}{2} \delta t[\vec{a}(t)+\vec{a}(t+\delta t)]
$$

In this algorithm the new velocities need the acceleration at time $t$ and $t+\delta t$. At first $\vec{r}(t+\delta t)$ can be calculated according to equation 2.6 using the velocities and acceleration 
at time $t$. The velocities at time $t+\frac{1}{2} \delta t$ can be obtained using following equation ${ }^{59}$

$$
\vec{v}\left(t+\frac{1}{2} \delta t\right)=\vec{v}(t)+\frac{1}{2} \delta t \vec{a}(t)
$$

At the end the velocity can be determined using

$$
\vec{v}(t+\delta t)=\vec{v}\left(t+\frac{1}{2} \delta t\right)+\frac{1}{2} \delta t \vec{a}(t+\delta t)
$$

Here the accelerations at time $t+\delta t$ are calculated from new forces based on current positions of particles.

\section{$2.2 \quad$ Force Field Models}

An empirical force field is an integral part of MD simulation. In molecular simulation, force field refers to the functional form of potential and parameter sets used to calculate the potential energy of a system of atoms or molecules. Force field parameters can be developed using quantum mechanical calculation or by reproducing different thermodynamical properties from experiments. The parameter sets can be classified into all-atom and united-atom, depending on how the interatomic potentials treat the atoms. All-atom force fields provide potential parameters corresponding to all atoms. United-atom force fields provide potential parameters corresponding to a group of atoms (like $\mathrm{CH}, \mathrm{CH}_{2}$, $\mathrm{CH}_{3}$ ). An equation for the force field for molecular simulation usually has four terms. Some sophisticated force fields have more terms but they have these four components in common. These four terms are divided into bonded interaction terms and nonbonded interaction terms. The bonded interaction terms are related to bond stretching, angle stretching and bond rotation. The nonbonded interaction term is a combination of Coulomb interaction and Van der Waals interaction. These four terms are responsible for the change of specific internal coordinates of atoms such as angle, bond length, rotation of bonds and movement of atoms, separately. This makes it simple to understand different effects due to different force field parameters. The equation corresponding to a simple 
force field for a single molecule (or for a group of molecules) is ${ }^{61,63-67}$

$$
\begin{aligned}
\mathcal{V}(r)=\sum_{\text {bonds }} \frac{k_{i}^{b}}{2}\left(b_{i}-b_{i, 0}\right)^{2}+ & \sum_{\text {angles }} \frac{k_{i}^{\theta}}{2}\left(\theta_{i}-\theta_{i, 0}\right)^{2}+\sum_{\text {dihedrals }} \frac{V_{n}}{2}(1+\cos (n \phi-\gamma))+ \\
& \sum_{i=1}^{N} \sum_{j=i+1}^{N}\left(4 \epsilon_{i j}\left[\left(\frac{\sigma_{i j}}{r_{i j}}\right)^{12}-\left(\frac{\sigma_{i j}}{r_{i j}}\right)^{6}\right]+\frac{q_{i} q_{j}}{4 \pi \epsilon_{0} r_{i j}}\right)
\end{aligned}
$$

Where $\mathcal{V}(r)$ is the potential energy which depends on the positions of particles. The first
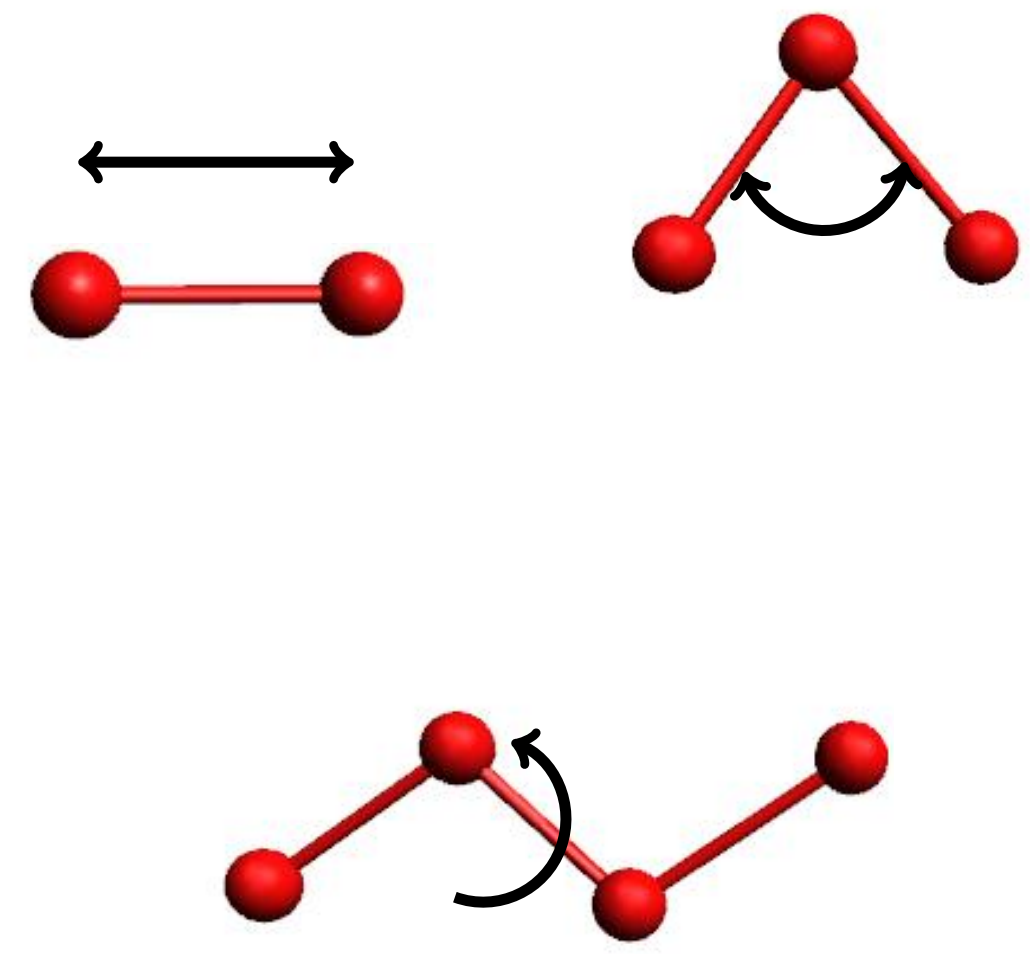

Figure 2.1: Sketch of bond stretching (top left), angle bending (top right) and torsional rotation (bottom).

term in the force field equation denotes the interaction between two bonded atoms (see figure 2.1, top-left). In the simple force field, this component is a harmonic potential and provides an increase in energy as the bond length $b_{i}$ deviates from its equilibrium value $b_{i, 0}$. The second term is a sum over all valence angles of bonded atoms (see figure 2.1 , top-right) and this term is also often modeled by a harmonic potential that gives an increase in energy as the angle $\theta_{i}$ deviates from the equilibrium value $\theta_{i, 0}$. The third term is the dihedral potential which measures the energy corresponding to bond rotation (see 
figure 2.1, bottom). The fourth term is nonbonded interactions between atoms. This is the interaction potential term between the atoms which are not bonded or separated by at least 3 bonds. The term has two parts; one is the Lennard--Jones potential part, describing Van der Waals interactions and another one is Coulomb electrostatic interaction part.

\subsubsection{Bonded Interactions}

\section{Bond stretching}

Different functional forms of potential terms are available to describe the bond stretching. One such function suggested by Morse is the most accurate. The function can be written $\operatorname{as}^{64,68}$

$$
\mathcal{V}_{\text {bond }}(b)=D_{e}\left(1-e^{-a\left(b-b_{0}\right)}\right)^{2}
$$

Here $b$ is the distance between the atoms, $b_{0}$ is the equilibrium bond distance, $D_{e}$ is the depth of potential energy minimum and " $a$ " controls the potential well width. Small " $a$ " means large well; $a=\sqrt{\frac{K_{e}}{2 D_{e}}}$, where $K_{e}$ is the force constant at the minimum of the well depth. This potential form of bond stretching is not usually used in MD simulation because it is computationally inefficient and also the function needs three parameters for each bond to specify. In most simulations simple forms of bond potential are used. One simple function is Hooke's law, in which potential energy is proportional to the square of the deviation of the bond length $(b)$ from the equilibrium bond length $\left(b_{0}\right)^{64,69}$

$$
\mathcal{V}_{\text {bond }}(b)=\frac{K^{b}}{2}\left(b-b_{0}\right)^{2}
$$

This functional form (2.14) of the potential is an approximation, and it gives accurate potential energy for bond stretching when bond stretching is small. It gives less accurate potential energy for bond stretching when the bond length $(b)$ is further away from the equilibrium value $b_{0}$. To get more accurate potential energy corresponding to bond stretching, the following formula can be used ${ }^{64,70}$

$$
\mathcal{V}_{\text {bond }}(b)=\frac{K^{b}}{2}\left(b-b_{0}\right)^{2}\left[1-K_{1}^{b}\left(b-b_{0}\right)-K_{2}^{b}\left(b-b_{0}\right)^{2}-K_{3}^{b}\left(b-b_{0}\right)^{3} \ldots \ldots \ldots \ldots\right]
$$


In the simulations of functionalized GNPs, equation 2.14 has been used as the potential energy function for the bond stretching.

\section{Angle Bending}

Usually, the harmonic functional form is used to calculate the potential energy contribution of angle bending during the simulation ${ }^{64}$

$$
\mathcal{V}_{\text {angle }}(\theta)=\frac{K^{\theta}}{2}\left(\theta-\theta_{0}\right)^{2}
$$

The potential energy contribution of angle bending is lower than that of bond stretching. To get a more accurate potential contributions from angle bending, the following function can be used ${ }^{64,70}$

$$
\mathcal{V}_{\text {angle }}(\theta)=\frac{K^{\theta}}{2}\left(\theta-\theta_{0}\right)^{2}\left[1-K_{1}^{\theta}\left(\theta-\theta_{0}\right)-K_{2}^{\theta}\left(\theta-\theta_{0}\right)^{2}-K_{3}^{\theta}\left(\theta-\theta_{0}\right)^{3} \ldots \ldots \ldots . .\right]
$$

Addition of higher order terms to the equation 2.16 gives more accuracy to the potential term. For simplicity and efficiency, equation 2.16 has been used in this work.

\section{Torsional Rotation}

Deformation of a torsional angle needs less energy than a bond stretching or angle bending. The torsional potential function represents steric barriers between the atoms separated by 3 covalent bonds. Several functional forms of torsional rotation potential are available, and all of them are cosine functions. One of the torsional potential expressions is ${ }^{71}$

$$
\mathcal{V}_{\text {torsion }}(\phi)=\sum_{n} \frac{V_{n}}{2}(1+\cos (n \phi-\gamma))
$$

The term $V_{n}$ gives the barrier height and $\mathrm{n}$ is the multiplicity which indicates the number of minimum points in the torsional potential function as the dihedral angle rotates from 0 to $360^{\circ}$. Another similar expression to equation 2.18 is ${ }^{64}$

$$
\mathcal{V}_{\text {torsion }}(\phi)=\sum_{k} A_{k} \cos (\phi)^{k}
$$


The potential function of torsion rotation, which is used in LAMMPS for the simulations of GNPs, is ${ }^{72}$

$$
\mathcal{V}_{\text {torsion }}(\phi)=\frac{K_{1}^{d}}{2}[1+\cos (\phi)]+\frac{K_{2}^{d}}{2}[1-\cos (2 \phi)]+\frac{K_{3}^{d}}{2}[1+\cos (3 \phi)]
$$

Where $K_{1}^{d}, K_{2}^{d}$ and $K_{3}^{d}$ are the dihedral coefficients. The first term of equation 2.20 corresponds to the interaction between bond dipoles, the second term corresponds to conjugation and hyperconjugation effects and the third term corresponds to steric interactions between the 1,4 atoms of the dihedrals. ${ }^{64}$

\subsubsection{Nonbonded Interactions}

Nonbonded interactions between pairs of atoms or molecules play an important role for the determination of the motion and structure of the molecules. Nonbonded interactions have two parts namely i) Coulomb interactions or electrostatic interactions and ii) van der Waals interactions. These two parts will be discussed in this subsection in details.

\section{Coulomb Interactions}

Coulomb interactions always refer to the interaction between charges. Charge can be distributed in different ways in a molecule. One way is to assign point charges throughout the molecule. If the charges are restricted to the nuclear center of atoms, then the charges are called partial atomic charges or net atomic charges. The Coulomb interaction or electrostatic interaction between two molecules with partial atomic charge can be calculated by the following equation ${ }^{64}$

$$
\mathcal{V}_{\text {electrostatic }}(r)=\sum_{i}^{N_{1}} \sum_{j \neq i}^{N_{2}} \frac{q_{i} q_{j}}{4 \pi \epsilon_{0} r_{i j}}
$$

$q_{i}$ and $q_{j}$ are the partial atomic charges of the atoms, $N_{1}$ and $N_{2}$ are the number of partial atomic charge of the molecules, and $r_{i j}$ is the distance between the partial atomic charges. An alternative way to calculate the electrostatic interaction is the central multipole expansion. This approach is computationally very efficient at large distances. In this approach, each molecule is considered a single entity. Using the multipole expansion 
approach, the expression of electrostatic interaction potential can be written as ${ }^{64,73,74}$

$$
\mathcal{V}_{\text {multipole }}(r)=\frac{q_{1} q_{2}}{4 \pi \epsilon_{0} r}+V_{\text {monopole-dipole }}(r)+V_{\text {dipole-dipole }}(r)+V_{\text {charge-quadrupole }}(r)+\ldots .
$$

$q_{1}$ and $q_{2}$ are the net charges of the molecules and $r$ is the distance between the different

poles. $V_{\text {monopole-dipole }}, V_{\text {dipole-dipole }}$ and $V_{\text {charge-quadrupole }}$ are interaction potential terms between monopole and dipole, dipole and dipole, and charge and quadrupole, respectively. The other approaches to calculate the electrostatic interaction are beyond the scope of this work. For the GNP simulations, equation 2.21 has been used in LAMMPS.

\section{Van der Waals Interactions}

Apart from the Coulomb interaction, one of the widely used nonbonded interactions is van der Waals interaction. For the calculation of van der Waals interaction, an accurate and computationally efficient expression is required. A well-known model is the LennardJones 12-6 function and the expression of the function is as follows ${ }^{64}$

$$
\mathcal{V}(r)=4 \epsilon\left[\left(\frac{\sigma}{r}\right)^{12}-\left(\frac{\sigma}{r}\right)^{6}\right]
$$

where $\epsilon$ is the well depth, $\sigma$ is the collision diameter and $r$ is the distance between the particles. This expression can be written in different form using $r_{0}$, where $r_{0}$ is the distance at which the potential reaches its minimum. The first derivative of energy with respect to the intermolecular distance $\left(\frac{\partial \mathcal{V}}{\partial r}=0\right)$ will provide $r_{0}=2^{1 / 6} \sigma$. The expression of Lennard-Jones 12-6 potential can be written as

$$
\mathcal{V}=\frac{A}{r^{12}}-\frac{B}{r^{6}}
$$

Where $\mathrm{A}$ is equal to $\epsilon r_{0}^{12}$ and $\mathrm{B}$ is equal to $2 \epsilon r_{0}^{6}$. The potential expression has two parts. One is the repulsive part which varies as $r^{-12}$ and the other is attractive part which varies as $r^{-6}$. This potential form is widely used for the van der Waals interaction energy calculation of big systems. Calculation of $r^{-12}$ and $r^{-6}$ are easy and that can be done without performing a computationally expensive square root calculation. To get less steep potential curve from the repulsive part of the equation, $r^{-12}$ can be replaced by $r^{-9}$ or $r^{-10}$. In some formulations $r^{-12}$ of the equation is replaced by a theoretically more 
accurate exponential expression. The new expression is as follows ${ }^{64}$

$$
\mathcal{V}(r)=\epsilon\left[\frac{6}{\alpha-6} \exp \left[-\alpha\left(r / r_{0}-1\right)\right]-\frac{\alpha}{\alpha-6}\left(\frac{r_{0}}{r}\right)^{6}\right]
$$

where $\alpha$ is a free dimensionless parameter. In the simulation of GNPs, equation 2.23 has been used in LAMMPS.

\subsection{Thermostating and Barostating}

Molecular dynamics is traditionally performed in the microcanonical ensemble (NVE). Most experiments in the real world are performed at constant temperature or pressure. To make the simulated system comparable to the real experiment, commonly MD simulations are nowadays performed in the NVT or in the NPT ensemble. Although it is possible to adjust the temperature of a system to a desirable value during the equilibration in the microcanonical ensemble. The NVT or NPT ensembles are important to study different properties of a system with respect to temperature or pressure.

\subsubsection{Thermostat}

A variety of thermostats are available to control the temperature of a system during a simulation. Popular methods to control the temperature are velocity rescaling, Nosé-Hoover thermostat, Berendsen thermostat, Anderson thermostat and Langevin thermostat. Here, only velocity rescaling, the Berendsen thermostat and the Nosé-Hoover thermostat techniques will be discussed.

\section{Velocity rescaling}

Velocity rescaling is the most simple method to keep the temperature of a system constant during a simulation. The temperature of a system is related to the time average kinetic energy of the system. For an unconstrained system the time average kinetic energy can be written as ${ }^{64}$

$$
\left\langle E_{k}\right\rangle_{N V T}=\frac{3}{2} N K_{b} T=\sum_{i=0}^{N} \frac{1}{2} m_{i} v_{i}^{2}
$$


If the temperature of the system at a time $t$ is $T(t)$ and the velocities are multiplied by a factor $\lambda$ to get a desired temperature $T_{d}$, then the temperature change of the system can be written as ${ }^{64}$

$$
\begin{gathered}
T_{d}-T(t)=\left(\lambda^{2}-1\right) T(t) \\
\lambda=\sqrt{T_{d} / T(t)}
\end{gathered}
$$

The easiest way to control the temperature is velocity rescaling by the factor of $\lambda$.

\section{Berendsen Thermostat}

The main problem of velocity rescaling is that it does not allow any fluctuation in temperature. To overcome this problem and to maintain the temperature of the system, Berendsen and coworkers ${ }^{75}$ introduced an external heat bath which is fixed at the desired temperature. The heat bath can add or remove thermal energy from the system to maintain the system temperature at the desired level. The velocities are scaled at each time step in such a way, that the rate of change of temperature is proportional to the difference between the heat bath temperature and the system temperature.

$$
\frac{d T(t)}{d t}=\frac{1}{\tau}\left(T_{r}-T(t)\right)
$$

Where $T_{r}$ is the temperature of the heat bath or heat reservoir and $\tau$ is the coupling factor. The value of $\tau$ defines how strongly the heat reservoir is coupled with the system. Large $\tau$ value makes coupling weak and small $\tau$ value makes coupling strong. If the value of $\tau$ becomes equal to the time step value $(\Delta t)$, then this technique will be equivalent to the velocity rescaling. The velocity scaling factor can be written as ${ }^{64}$

$$
\lambda^{2}=1+\frac{\Delta t}{\tau}\left(\frac{T_{r}}{T(t)}-1\right)
$$

This method produces an exponential decay towards the temperature of the heat bath. The beauty of this method is that it allows temperature fluctuations, which are present in the canonical ensemble. However, the method has some drawbacks. One drawback is that the technique is time irreversible or deterministic and an other disadvantage is that it does not remove localized or unwanted correlation motion of particles in the system. This method is not recommended for production runs because it does not strictly conform 
to the canonical ensemble, but it can be used during equilibration.

\section{Nosé-Hoover Thermostat}

The stochastic collision method and the extended system method are two well known methods which can generate a proper canonical ensemble. In the stochastic collision method a particle inside the system is selected randomly at a certain interval and its velocity is modified by random selection from the Maxwell-Boltzmann distribution. In the extended system method, the heat reservoir is considered as an integral part of the system and this method is known as Nosé-Hoover thermostat. The Nosé-Hoover thermostat is the default thermostat for the NVT ensemble in the LAMMPS simulation package. The heat reservoir in this method is introduced by adding an additional degree of freedom in the Lagrangian of the system. The Lagrangian of a classical N-body system with the additional degree of freedom is ${ }^{62}$

$$
\mathcal{L}_{\text {Nose-Hoover }}=\sum_{i=1}^{N} \frac{m_{i}}{2} s^{2} \dot{\overrightarrow{r_{i}}}-\Phi(\vec{r})+\frac{Q}{2} \dot{s}^{2}-\frac{g}{\beta} \ln s
$$

where $s$ is a parameter that scales the velocities, $Q$ is an effective mass associated to $s$ and $\mathrm{g}$ is equal to the degrees of freedom of the system. The Hamiltonian of the corresponding system is

$$
\mathcal{H}_{\text {Nose-Hoover }}=\sum_{i=1}^{N} \frac{{\overrightarrow{p_{i}}}^{2}}{2 m_{i} s^{2}}+\Phi(\vec{r})+\frac{p_{s}^{2}}{2 Q}+\frac{g}{\beta} \ln s
$$

Where, $\vec{p}_{i}$ is $m_{i} s^{2} \dot{\overrightarrow{r_{i}}}$ and $p_{s}$ is $Q \dot{s}$. From this Hamiltonian, the equations of motion can be derived easily. The mass $Q$ determines the strength of the thermostat, and the choice of $Q$ is critical.

\subsubsection{Barostat}

Often it is desirable to control the pressure of a simulated system. At constant pressure, the volume of a simulated system changes during the simulation. Different barostat methods are available for maintaining a desired pressure by changing the system volume. Many of the barostats are analogous to the thermostats, which were discussed in the previous subsection 2.3.1. Some well known barostat methods are volume rescaling, Berendsen 
barostat and extended ensemble barostat or Nosé-Hoover barostat.

\section{Berendsen Barostat}

The Berendsen barostat is one of the simplest methods to control the pressure of a system during a simulation. In this method the system is weakly coupled with an external constant pressure reservoir which is analogous to the way temperature is maintained in the Berendsen thermostat. The rate of pressure change is defined by ${ }^{76}$

$$
\frac{d P(t)}{d t}=\frac{1}{\tau_{p}}\left(P_{d}-P(t)\right)
$$

$P_{d}$ is the desired pressure, $P(t)$ is the system pressure at time $t$, and $\tau_{p}$ is the time constant of the barostat. To adjust this pressure change, the volume of the system is scaled by a factor $\mu^{3}$. So the coordinates of the particles change by a factor $\mu$. $\mu$ is

$$
\mu=\left[1-\frac{\Delta t}{\tau_{p}}\left(P(t)-P_{d}\right)\right]^{\frac{1}{3}}
$$

The strength of the coupling between the system and the pressure reservoir is determined by $\tau_{p}$.

\section{Nosé-Hoover Barostat}

This barostat is similar to the Nosé-Hoover thermostat. To create the barostat, the extended system method has been used. The equations of motion corresponding to this 
barostat $\operatorname{are}^{77}$

$$
\begin{gathered}
\dot{\overrightarrow{r_{i}}}=\frac{\vec{p}_{i}}{m_{i}}+\frac{p_{\epsilon}}{W} \vec{r}_{i} \\
\dot{\vec{p}}_{i}=\vec{F}_{i}-\frac{p_{\epsilon}}{W} \vec{p}_{i}-\frac{p_{\xi}}{Q} \vec{p}_{i} \\
\dot{V}=\frac{d V_{p_{\epsilon}}}{W} \\
\dot{p}_{\epsilon}=d V\left(P_{\text {in }}-P_{\text {out }}\right)-\frac{p_{\xi} p_{\epsilon}}{Q} \\
\dot{\xi}=\frac{p_{\xi}}{Q} \\
\dot{p}_{\xi}=\sum_{i=1}^{N} \frac{\vec{p}_{i}^{2}}{m_{i}}+\frac{p_{\epsilon}^{2}}{W}-\left(N_{f}+1\right) k T
\end{gathered}
$$

$\mathrm{V}$ is the volume of the system, $p_{\epsilon}$ is the barostat momentum, $\xi$ and $p_{\xi}$ are the thermostat position and momentum, $F_{i}$ is the force on $i$ th particle, $P_{i n}$ and $P_{\text {out }}$ are the internal and external pressure on the system, $W$ is the mass parameter associated with $\epsilon, Q$ is the coupling constant, and $N_{f}$ denotes the number of degrees of freedom.

\subsection{Water Model}

Different water models are used in MD simulations of liquid water, water clusters and aqueous solution. TIP3P is a widely used and popular water model in MD simulation for its simplicity and computational efficiency. It is a three-point rigid water model. Three-point indicates that three point charges are centered on the three atoms of the water molecule; the positive point charges on hydrogen atoms are exactly balanced by the negative charge on the oxygen atom. This model uses a pairwise interaction potential for the nonbonded atoms, whose form is a combination of Coulomb interaction potential and 12-6 Lennard--Jones potential. This potential combination is compatible with most well

known force fields. The interaction potential form of TIP3P is represented by equation ${ }^{78}$ 2.35 .

$$
\mathcal{V}_{a b}=\sum_{i}^{a} \sum_{j}^{b} \frac{K_{c} q_{i} q_{j}}{r_{i j}}+\frac{A}{r_{O O}^{12}}-\frac{B}{r_{O O}^{6}}
$$

Where $K_{c}$ is the electrostatic constant, $q_{i}$ and $q_{j}$ are the partial charges of atoms, $r_{i j}$ is the distance between two charge points, $A$ and $B$ are the Lennard-Jones parameters. 


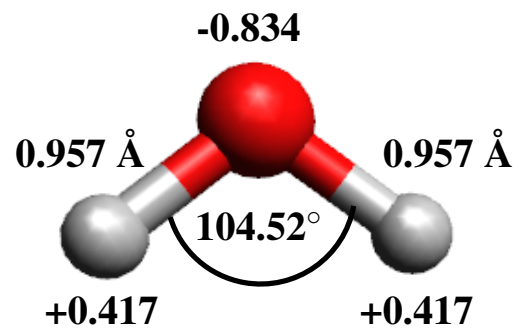

Figure 2.2: Sketch of TIP3P water model. Different numbers in the figure represent bond lengths, partial charge of atoms and $\mathrm{H}-\mathrm{O}-\mathrm{H}$ angle.

The TIP3P water model was developed to reproduce several key features of bulk water at $25^{\circ} \mathrm{C}$. These are the density of water $(0.997 \mathrm{~g} / \mathrm{mol})$, the heat of vaporization $(10.53$ $\mathrm{kcal} / \mathrm{mol}$ ), and the radial distribution function of oxygen atoms consistent with light scattering experiment ${ }^{79-81}$.

\subsection{Analysis Tools}

In this section some basic functions and analysis methods are defined and described. Other analysis methods are discussed in next chapters where the methods are used for the analysis of systems.

\subsubsection{Radial Distribution Function}

The radial distribution function ( $\mathrm{RDF}$ or $\mathrm{g}(\mathrm{r})$ ) is a basic means to describe the structure of a system and it is strongly connected to x-ray diffraction in experiment. The RDF shows how the density of particles varies as a function of distance from a reference particle. Actually, it represents the relative density of particles as a function of distance from a reference particle. It can be defined as the ratio of the density of particles at a distance $r$ from a particle in a system to the overall density of particles in that system. The RDF 


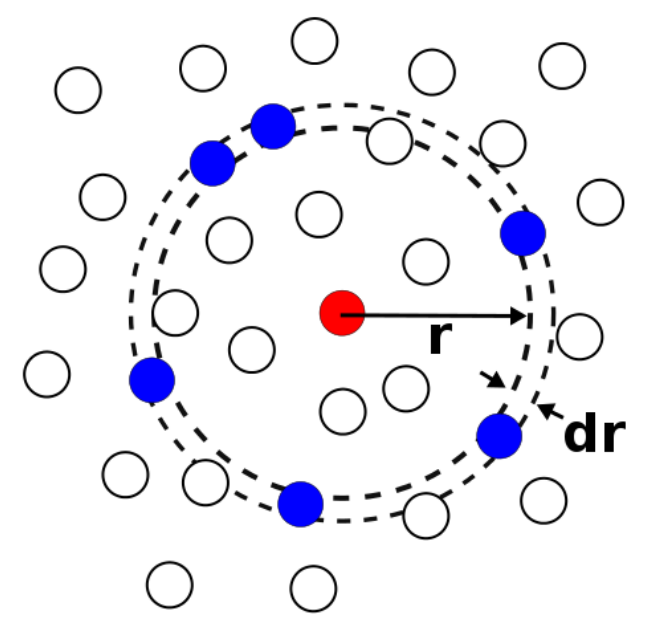

Figure 2.3: Sketch for the RDF. Particles are highlighted (blue) inside a shell $r$ and $r+d r$ with respect to a reference particle (red).

can be expressed by the following equation

$$
g_{i j}(r)=\frac{1}{4 \pi \rho_{j} r^{2} d r} \cdot \frac{N_{i j}(r)}{N_{i}}
$$

Where $N_{i j}(\mathrm{r})$ denotes the average number of particles of a species $j$ between spherical shells of radii $r$ and $r+d r$ around the particle $i$ (see figure 2.3). $\rho_{j}$ is the number density of $j$ type particles in the system, $d r$ is a small increment in radius, and $N_{i}$ is the total number of particles of the $i$ th species. The radial density $\left(R_{d}\right)$ can be calculated using equation 2.36. Considering only a single species and a reference point inside the system, the equation of $R_{d}$ is

$$
R_{d}=\frac{\delta N_{i j}(r)}{4 \pi \rho r^{2} d r}
$$

$\delta N_{i j}(r)$ is the number of particles inside a spherical shell of radii $r$ and $r+d r$ and $\rho$ is the density density of the particles inside the system.

\subsubsection{Center of Mass}

The center of mass $\left(\vec{R}_{c m}\right)$ of a distribution of particles can be regarded as the unique point in which the entire mass is concentrated. Alternatively, the center of mass can 
be regarded as the point with respect to which the sum of the weighted positions of the particles is always zero. If a system has $N$ particles of mass $m_{1}, m_{2}, m_{3} \ldots . m_{n}$ and positions $\vec{r}_{1}, \vec{r}_{2}, \vec{r}_{3} \ldots . \vec{r}_{n}$ with respect to the reference coordinate then the center of mass must satisfy the following condition

$$
\sum_{i=1}^{N} m_{i}\left(\vec{r}_{i}-\vec{R}_{c m}\right)=0
$$

Now, the center of mass is

$$
\vec{R}_{c m}=\frac{1}{M} \sum_{i=1}^{N} m_{i} \vec{r}_{i}
$$

Where $M$ is the total mass of all particles. For a continuous distribution of particles of density $\rho(\vec{r})$ inside a volume $\mathrm{V}$, the center of mass equation would be

$$
\vec{R}_{c m}=\frac{1}{M} \iiint_{V} \rho(\vec{r}) \vec{r} d v
$$

\subsubsection{Radius of Gyration}

The radius of gyration $\left(R_{g}\right)$ refers to the distribution of particles of a system around the center of mass of the system. The radius of gyration of an object can be calculated using the mass moment of inertia. In mechanics, the radius of gyration can be written as ${ }^{82}$

$$
R_{g}=\sqrt{\frac{I_{a x}}{M}}
$$

Where $I_{a x}$ is the mass moment of inertia around the axis and $M$ is the total mass of the object. In molecular physics, the radius of gyration of a system is the root mean square distance of the particles from the center of mass of the system. ${ }^{83}$

$$
R_{g}=\sqrt{\frac{\sum_{i} m_{i} r_{i}^{2}}{\sum_{i} m_{i}}}
$$

Where $m_{i}$ is the mass of the $i$ th particle and $r_{i}$ is the distance of the particle from the center of the system. In this work the radius of gyration has been calculated using 
equation 2.42 .

\subsubsection{Diffusion Coefficient}

The diffusion coefficient of a continuous system can be determined by Fick's law relating mass flow to density gradient ${ }^{58}$

$$
\rho \vec{v}=D \vec{\nabla} \rho
$$

Where $\rho(\vec{r}, \mathrm{t})$ is the local density and $\vec{v}(\vec{r}, \mathrm{t})$ is the local velocity. The time derivative of the local density is ${ }^{58}$

$$
\frac{\partial \rho}{\partial t}=D \nabla^{2} \rho
$$

For a system of $N$ particles, the local density $\rho$ can be defined as ${ }^{58}$

$$
\rho(\vec{r}, t)=\sum_{i=1}^{N} \delta\left(\vec{r}-r_{i}(t)\right)
$$

Equation 2.44 can be used for both the diffusion of species through another one and for self-diffusion within a phase of only a single species.

For larger $t$ than to the collision time between particles, Einstein's expression can be used to calculate the self diffusion coefficient. The mathematical form of Einstein's expression $^{58}$ is

$$
D=\lim _{t \rightarrow \infty} \frac{1}{6 N t}\left\langle\sum_{i=1}^{N}\left[\vec{r}_{i}(t)-\vec{r}_{i}(0)\right]^{2}\right\rangle
$$

Another way to calculate the self diffusion coefficient is by using the Green-Kubo equation. This method uses the velocity autocorrelation function. ${ }^{58}$

$$
D=\frac{1}{3 N} \int_{0}^{\infty}\left\langle\sum_{i=1}^{N} \vec{v}_{i}(t) \cdot \vec{v}_{i}(0)\right\rangle d t
$$

Equation 2.46 and 2.47 are very similar. For the calculation of diffusion coefficient, here equation 2.46 has been used. 


\subsubsection{Hydrogen Bonds}

A hydrogen bond is an interaction between a hydrogen atom which is bonded to a highly electronegative atom and a nearby highly electronegative atom (like fluorine, oxygen or nitrogen). In molecular force fields hydrogen bonds are usually described as electrostatic interactions.
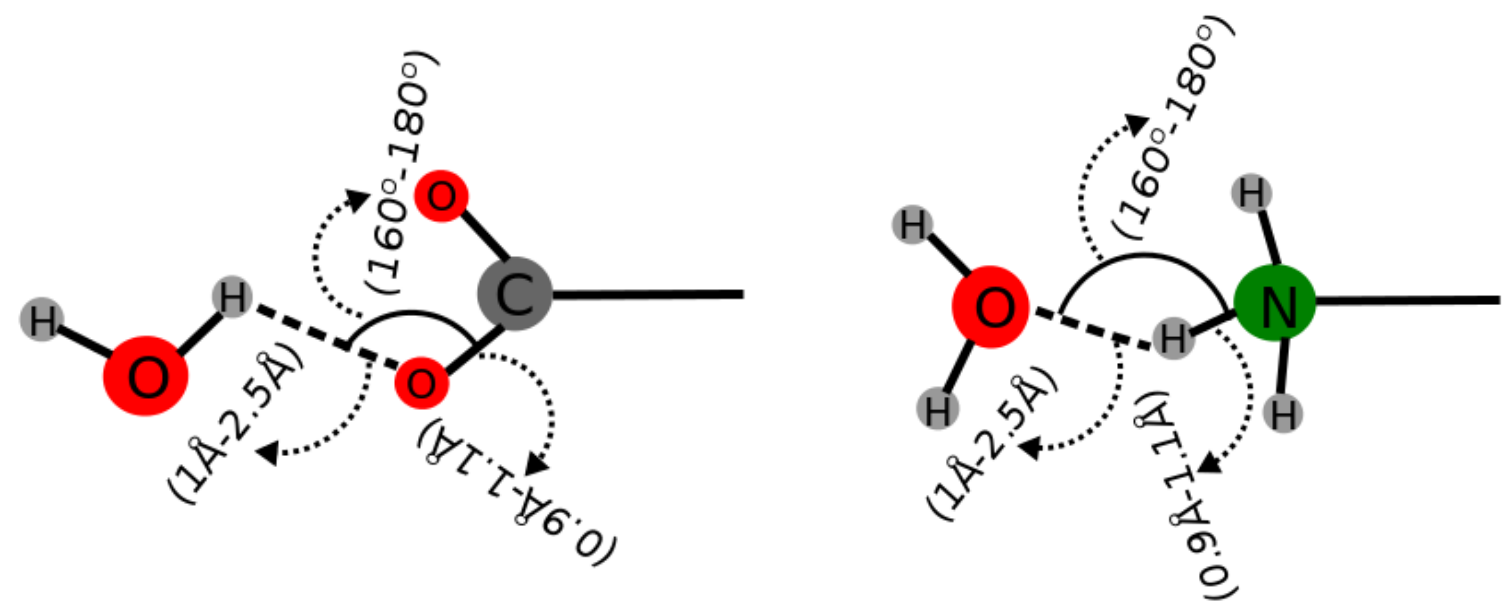

Figure 2.4: Left side: hydrogen bond between hydrogen of water molecule and oxygen of $\mathrm{COO}^{-}$terminal group. Right side: hydrogen bond between hydrogen of $\mathrm{NH}_{3}^{+}$terminal group and oxygen of water molecule

The criteria for the hydrogen bond between a $\mathrm{COO}^{-}$terminal group and water are (see left side of the figure 2.4):

1. The distance between the hydrogen of water and one oxygen atom of the $\mathrm{COO}^{-}$ terminal is $1 \AA-2.5 \AA$ ( $\mathrm{H}-\mathrm{O}$ distance). This has been decided considering the first peak of the RDF between the hydrogen of water and the oxygen atom of the $\mathrm{COO}^{-}$ terminal group.

2. The distance between $\mathrm{C}$ and $\mathrm{O}$ of terminal $\mathrm{COO}^{-}$is $0.9 \AA-1.1 \AA$.

3. The angle $\angle \mathrm{H}-\mathrm{O}-\mathrm{C}$ is $160^{\circ}-180^{\circ}$.

The criteria for the hydrogen bond between $\mathrm{NH}_{3}^{+}$terminal groups and water are (see right side of the figure 2.4): 
1. The distance between the hydrogen of the $\mathrm{NH}_{3}^{+}$terminal group and the oxygen atom of water is $1 \AA-2.5 \AA$ ( $\mathrm{H}-\mathrm{O}$ distance). This has again been decided considering the first peak of the RDF between the hydrogen of the $\mathrm{NH}_{3}^{+}$terminal and the oxygen atom of water.

2. The distance between $\mathrm{N}$ and $\mathrm{H}$ of terminal $\mathrm{NH}_{3}^{+}$is $0.9 \AA-1.1 \AA$.

3. The angle $\angle \mathrm{H}-\mathrm{O}-\mathrm{N}$ is $160^{\circ}-180^{\circ}$. 


\section{Cluster Formation of $\mathrm{NaCl}$ in Bulk Solutions}

The quality of MD simulation results is highly dependent on the accuracy and reliability of the force field parameters. Inaccurate force field parameters can lead to unphysical behavior of the simulated systems. Many force field parameter sets are available in the literature, but not all of them are useful for electrolyte solution systems at finite concentration due to inaccuracy in the parameters. To make the right decision on force field parameter sets for the present study, simulations of $\mathrm{NaCl}$ solution systems have been performed using different force field parameters. In this chapter, three different force fields namely, i) AMBER-99 ${ }^{84}$ ii) OPLS-AA ${ }^{85}$ and iii) CHARMM-27 ${ }^{86,71}$ are compared together with the TIP3P ${ }^{87}$ water model. In addition, two different combination rules, geometric and arithmetic (geometric mixing for $\epsilon$ and arithmetic mixing for $\sigma$ ) and three different concentrations are compared at $300 \mathrm{~K}$.

\subsection{Simulation Details}

15 different $\mathrm{NaCl}$ solution systems are prepared varying the solution densities, force fields and combination rules. Systems with three different concentrations of $1.1,1.5$ and 2.0 molal have been prepared. All simulation boxes have the same size of $80 \times 80 \times 80 \AA^{3}$ and contain 330, 460 and $613 \mathrm{Na}^{+}$and $\mathrm{Cl}^{-}$ions for the concentrations of $1.1,1.5$ and 2.0 molal, respectively. Initially boxes ware filled with water, then $\mathrm{Na}^{+}$and $\mathrm{Cl}^{-}$ions were placed using $\mathrm{VMD}^{88}$ (1.9.1), employing the TopoTool plugin. The numbers of water molecule and ions were adjusted to obtain the desired concentrations, and the minimum distances between the ions were $5 \AA$ initially. 
Force field parameters were taken from AMBER-99, CHARMM-27 and OPLS-AA force fields. Two combination rules, arithmetic and geometric, were used to generate parameters for both $\sigma$ (finite distance at which the inter-particle potential is zero) and $\epsilon$ (well depth) for the Lennard-Jones (L-J) interaction parameters between all ion pairs. The TIP3P water model ${ }^{87}$ was used to ensure consistency among force field parameters. All the force field parameters used for the simulations are given in Table 3.1.

Table 3.1: Force field parameters

\begin{tabular}{|c|c|c|c|c|}
\hline \multirow{2}{*}{ Force field } & \multicolumn{2}{|c|}{$\mathrm{Na}^{+}$} & \multicolumn{2}{c|}{$\mathrm{Cl}^{-}$} \\
\cline { 2 - 5 } & $\sigma[\AA]$ & $\epsilon[\mathrm{kcal} / \mathrm{mol}]$ & $\sigma[\AA]$ & $\epsilon[\mathrm{kcal} / \mathrm{mol}]$ \\
\hline AMBER-99 & 3.3284 & 0.00277 & 4.40104 & 0.1000 \\
OPLS-AA & 3.3304 & 0.00280 & 4.41720 & 0.1178 \\
CHARMM-27 & 2.4299 & 0.04690 & 4.04470 & 0.1500 \\
\hline
\end{tabular}

The LAMMPS ${ }^{89}$ (version 31, March, 2011) molecular dynamics package is employed to simulate all systems. Temperatures, volumes and the number of particles were held constant (NVT) during the simulations. The volume of the systems was held constant to avoid the fluctuation in density of the solutions. A Nosé-Hoover thermostat ${ }^{59}$ with a time constant of 1 ps was used to keep the system temperature constant at $300 \mathrm{~K}$. The LennardJones interactions were cut off at a distance of $10 \AA$ and the SHAKE $^{90}$ algorithm was used to constrain O-H bonds and H-O-H angles. The particle-particle-particle-mesh (PPPM) method has been used with an accuracy (relative error in per-atom forces calculation) of $10^{-4}$ to compute long range Coulomb interactions. Several short runs of 100 ps have been performed with time steps of less than 1 fs to come close to equilibrium quickly. Production runs were performed with a time step of $1 \mathrm{fs}$ and a skin distance for the neighbor tables of $0.1 \mathrm{~nm}$, which was updated every 5 steps. Systems were equilibrated for $\approx 5 \mathrm{~ns}$ and all analysis has been performed over 20 ns production runs.

\subsection{Results and Discussion}

Snapshots in Figure 3.1 show the $\mathrm{Na}^{+}$and $\mathrm{Cl}^{-}$ions of a system (AMBER-1.5m-arithmetic) at 1.5 molal concentration. For this system AMBER-99 force field parameters were used for ions and the arithmetic combination rule was used for the L-J interaction parameters of ion pairs. The left side of the snapshots shows the initial state of the system at 0 ns 

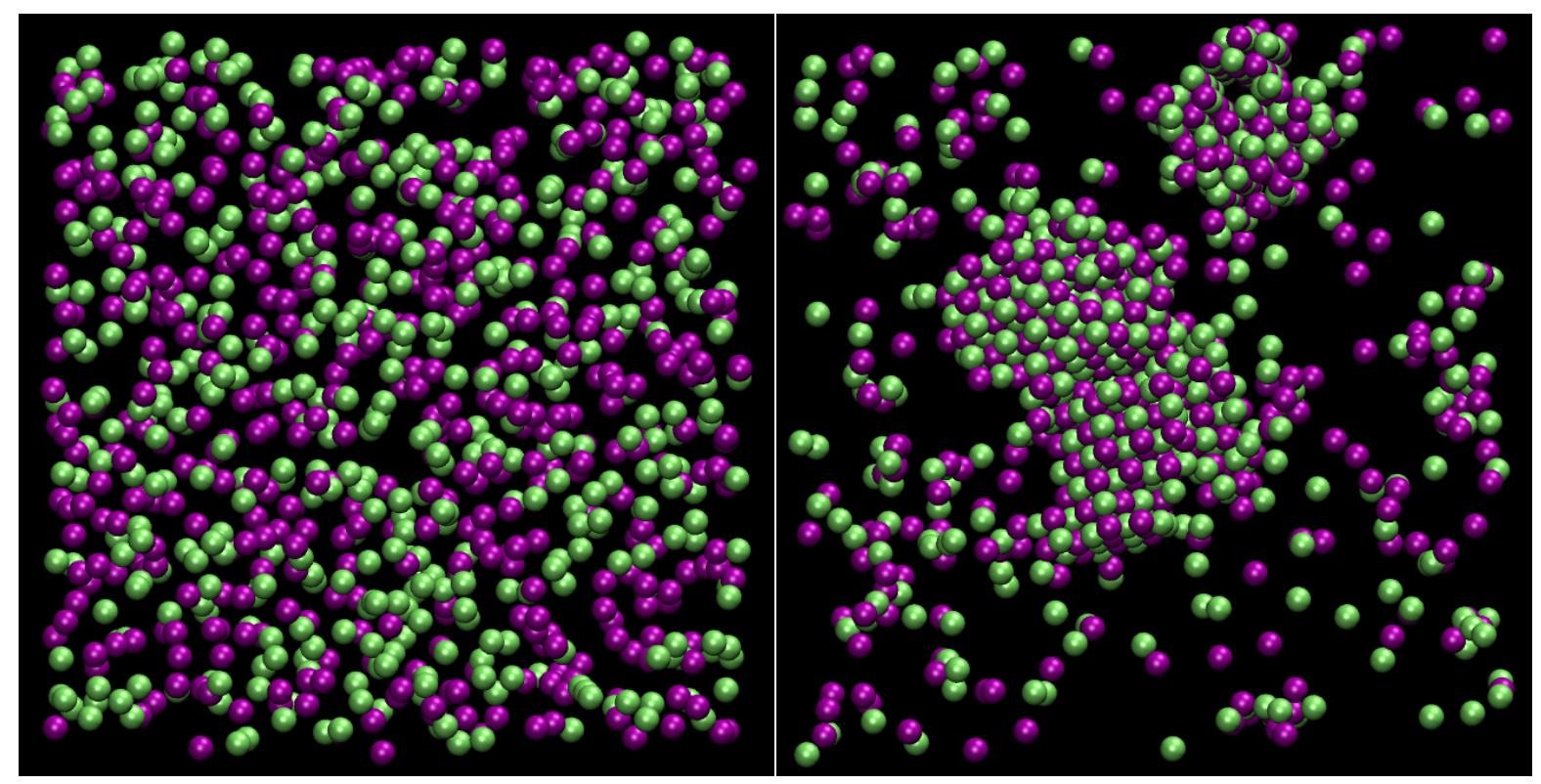

Figure 3.1: Snapshots of a 20 ns long simulation of $\mathrm{NaCl}$ solvated in TIP3P water (not shown here) at a concentration of 1.5 molal using AMBER-99 ion parameters. Initial configuration (left) at $0 \mathrm{~ns}$ and final configuration (right) at $20 \mathrm{~ns}$; $\mathrm{Na}^{+}$and $\mathrm{Cl}^{-}$are shown in green and pink, respectively.

and the right side shows the final state of the system at 20 ns. The visual examination clearly shows the rapidity of the aggregation process, which leads to the formation of large crystalline clusters within 20 ns. Within 20 ns most ions aggregate to form clusters, leaving few ions unpaired. These clusters are face centered cubic, like $\mathrm{NaCl}, \mathrm{KCl}$ or $\mathrm{LiF}$ crystals.

Figure 3.2 shows the radial distribution function between $\mathrm{Na}^{+}$and $\mathrm{Cl}^{-}$for the systems where the AMBER-99 force field parameters were used. All radial distributions are averaged over $5 \mathrm{~ns}$ blocks of the $20 \mathrm{~ns}$ simulations. Strong oscillations at distances between 4 to $14 \AA$ indicate 3 -D crystal formation in the systems. All systems with arithmetic combination rule (left column) show indications of crystallization within 20 ns. Aggregation starts for AMBER-1.1m-arithmetic (top left) and AMBER-1.5m-arithmetic (center left) systems after 15 and $5 \mathrm{~ns}$, respectively. Crystallization is slow at low concentration and its rate increases with increasing concentration. Systems with AMBER-99 parameters and geometric combination rule (right column) do not show any indication of crystallization up to 1.5 molal (right center) but the system with high concentration of 2.0 molal (bottom right) shows crystallization, although the concentration of solutions are much lower than 

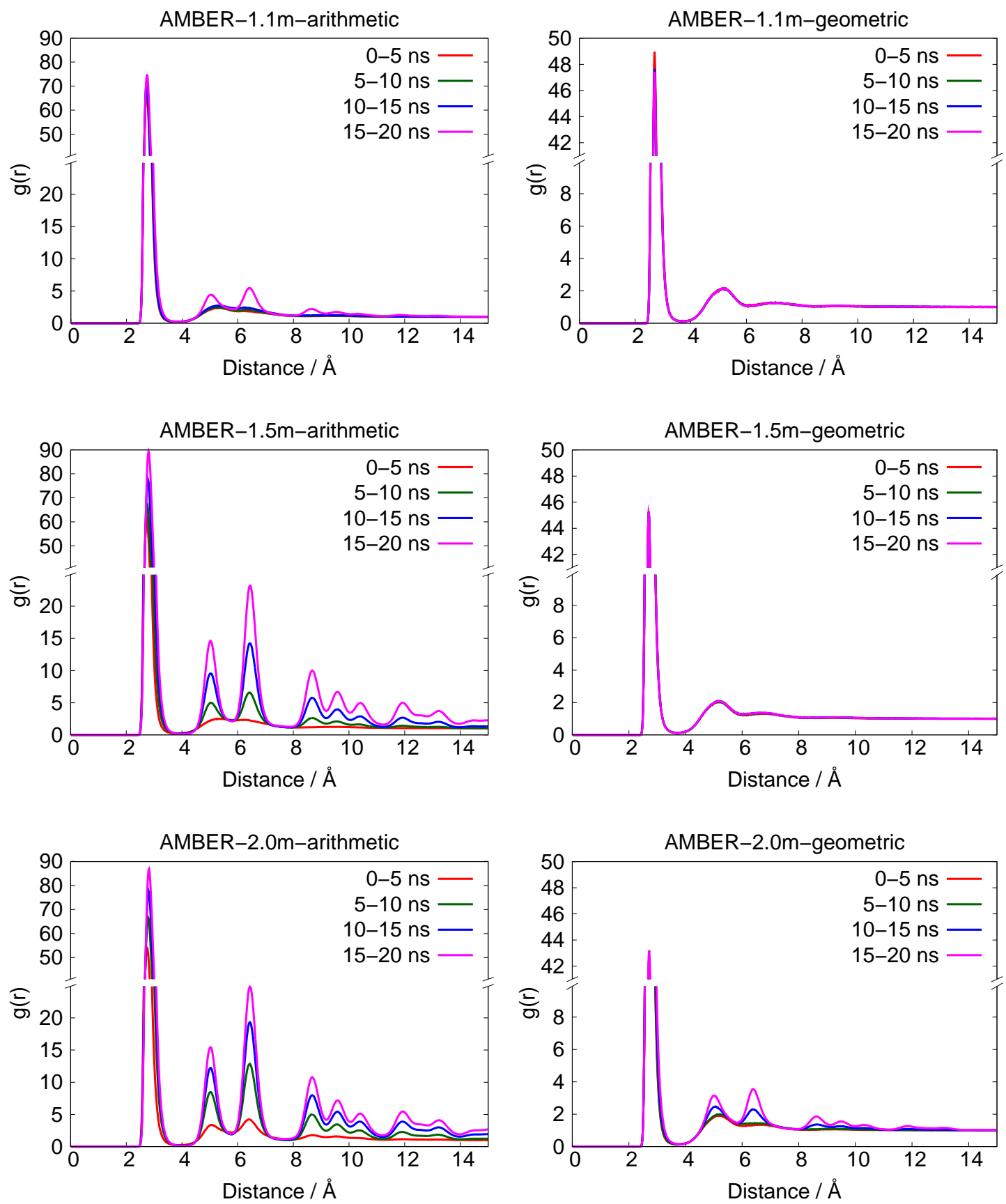

Figure 3.2: Na-Cl radial distribution functions calculated over $5 \mathrm{~ns}$ time interval of $20 \mathrm{~ns}$ long simulations. In left and right column, systems have different concentrations 1.1, 1.5 and 2.0 molal (top to bottom). Different multiplication rules, as arithmetic (left column) and geometric (right column) used for cross force field parameters. 
the experimental solubility level of 5.4 molal $^{91}$ at $293 \mathrm{~K}$.

AMBER-99 together with the arithmetic combination rule is unsuitable for $\mathrm{NaCl}$ solution systems due to the crystallization phenomenon. This unphysical behavior of ions arises due to the shortcomings in parametrization of ions in AMBER-99. AMBER99 adopted cation parameters form Åqvist ${ }^{92}$. Åqvist employed geometric combination rules for ion pairs. The use of geometric combination rules instead of arithmetic ones in AMBER-99 improves the solubility of ions in solution, but nevertheless ions start to crystallize at high concentration.

Figure 3.3 shows $\mathrm{Na}-\mathrm{Cl}$ coordination numbers of all 6 sets of simulations with OPLSAA using different combination rules. Cutoff distances of coordination numbers are decided considering the positions of minima of RDFs. First, second and third coordination numbers are calculated by averaging all $\mathrm{Cl}^{-}$ions around $\mathrm{Na}^{+}$ions in the ranges of 2.6 $\AA-3.6 \AA, 4.6 \AA-5.6 \AA$ and $6 \AA-7 \AA$, respectively. The coordination numbers of OPLS-AA with arithmetic combination rules at the higher concentrations of $1.5 \mathrm{M}$ (left center) and $2.0 \mathrm{M}$ (left bottom) show a continuous rise of the values with time but much less so the ones at low concentration of $1.1 \mathrm{M}$ (left top). So, at high concentrations, the systems form crystals and the continuous growth of numbers indicates that the crystal formation is still incomplete. In long runs, systems are likely to form big crystals leaving few ions unpaired. The coordination numbers do not increase with increasing time for simulations with the OPLS-AA force field and geometric combination rules (right column). The coordination numbers remain constant throughout the simulations irrespective of concentrations.

These results show that the OPLS-AA force field with arithmetic combination rule is not useful for the $\mathrm{NaCl}$ solution simulation. Short simulation times could give erroneous informations about the system. The OPLS-AA force field with geometric combination rules shows the expected behavior throughout all simulations. So, OPLS-AA with geometric combination rules is useful for the simulation up to about 2.0 molal density.

Figure 3.4 displays the $\mathrm{Na}-\mathrm{Cl}$ coordination numbers over 5 ns time intervals of 20 ns long simulations of CHARMM-27 with arithmetic combination rule. In this figure, coordination numbers do not change over time even at the high concentration of 2.0 molal (right). So, the systems with CHARMM-27 ions parameters and arithmetic combination rule do not show any crystallization. Hence, this parameter set and the combination rules can be used for simulation of aqueous solution of $\mathrm{NaCl}$ system.

All 15 systems are categorized in table 3.2, depending on the crystallization. '十' 

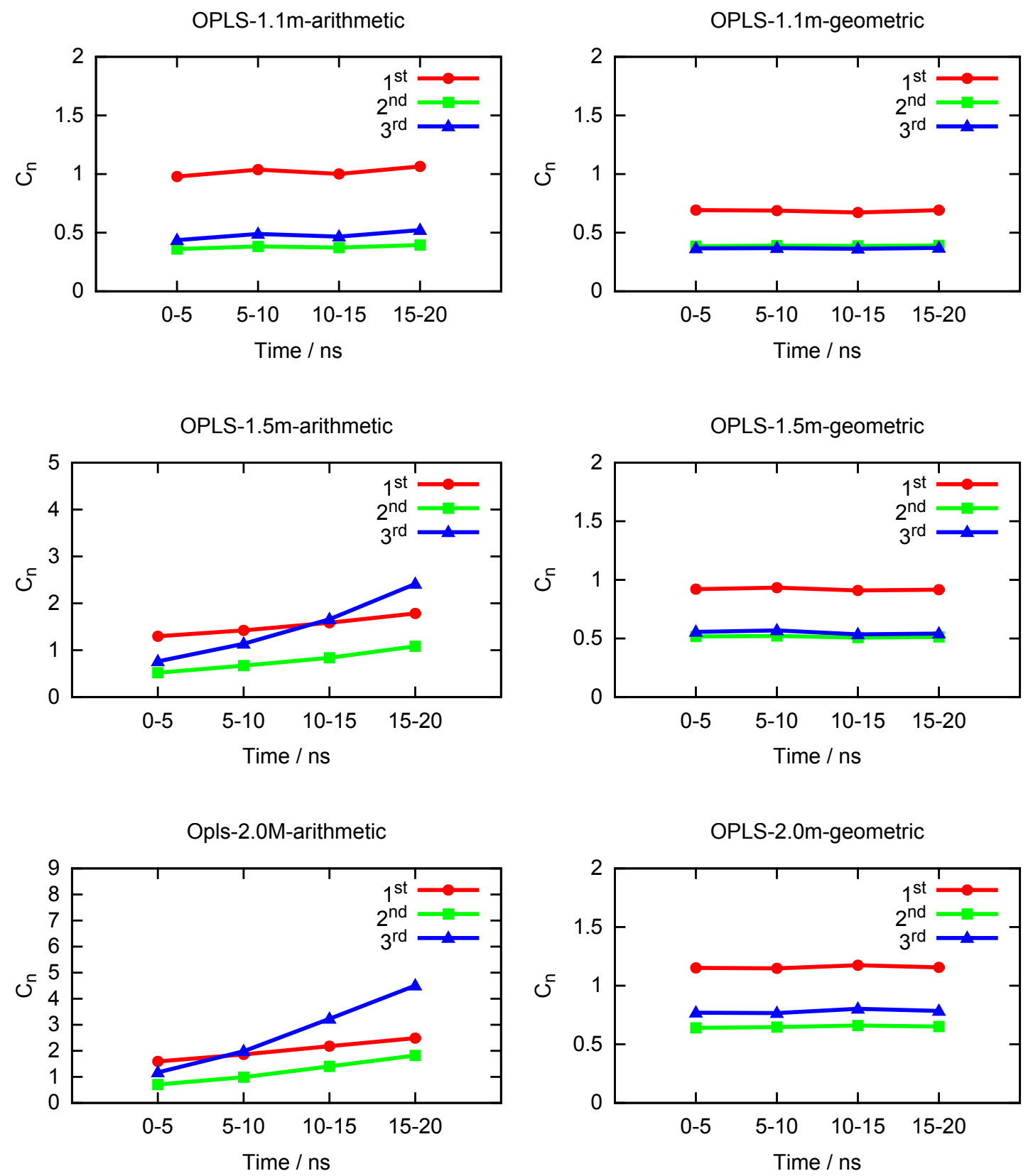

Figure 3.3: $\mathrm{Na}-\mathrm{Cl}$ coordination numbers are $\left(\mathrm{C}_{n}\right)$ calculated by averaging over 5 ns interval of $20 \mathrm{~ns}$ long runs. Arithmetic (left column) and Geometric (right column) production rules are used for cross force field parameters. Red, green and blue line points shows 1st, 2nd and 3rd coordination, respectively. 

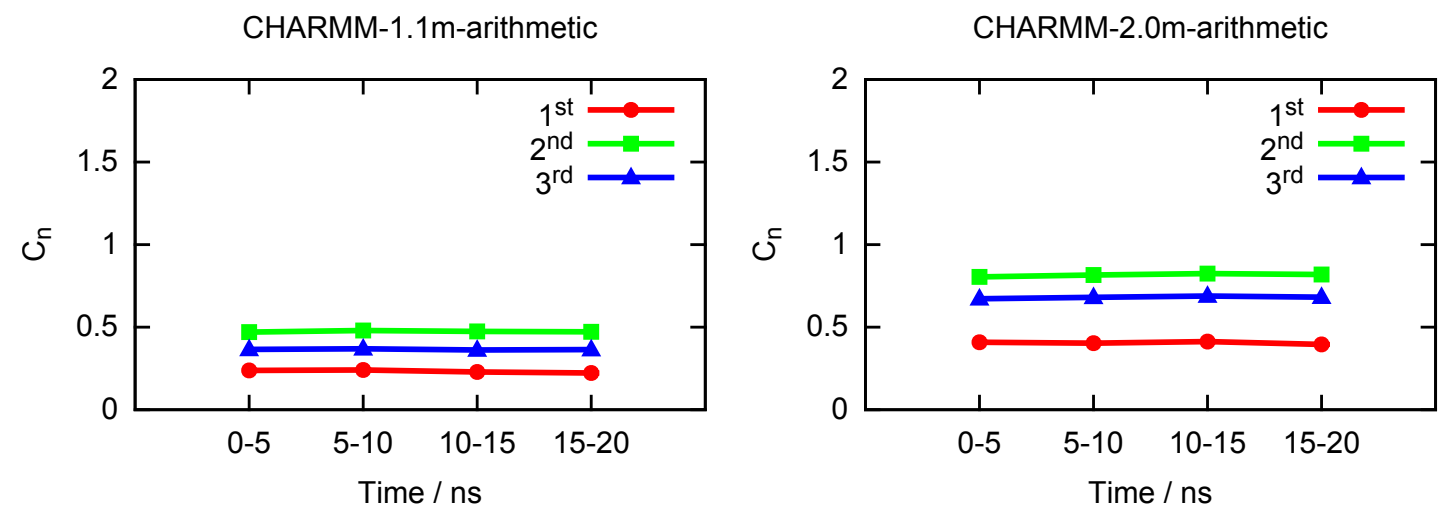

Figure 3.4: Na-Cl coordination numbers are calculated by averaging over 5 ns interval of $20 \mathrm{~ns}$ long runs. Left side is for 1.1 molal and the right side is for 2.0 molal concentrations.

signs in the table indicate that ions form crystals and '-' signs indicate that the system does not form crystals during the time interval of simulations.

Table 3.2: Comparison of force fields

\begin{tabular}{|c|c|cccc|cc|}
\hline \multirow{2}{*}{ Density (mol/l) } & Time (ns) & \multicolumn{5}{|c|}{ Combination Rules } \\
\cline { 2 - 7 } & & \multicolumn{4}{|c|}{ Arithmetic } & \multicolumn{2}{c|}{ Geometric } \\
\cline { 2 - 7 } 1.1 & 5 & Amber & Charmm & Opls & Amber & Opls \\
\hline \multirow{3}{*}{1.5} & 10 & - & - & - & - & - \\
& 15 & - & - & - & - & - \\
& 20 & + & - & - & - & - \\
& 5 & - & - & - & - & - \\
& 10 & + & - & + & - & - \\
& 15 & + & - & + & - & - \\
& 20 & + & - & + & - & - \\
\hline \multirow{3}{*}{2.0} & 5 & + & - & - & - & - \\
& 10 & + & - & + & - & - \\
& 15 & + & - & + & + & - \\
& 20 & + & - & + & + & - \\
\hline
\end{tabular}

Finally, the decision has been made to use the popular force field AMBER-99 for the functionalized gold nanoparticle simulations. Based on the findings, to overcome the crystallization phenomena, AMBER-99 force field parameters of ions are replaced by ion parameters from the CHARMM-27 force field. 


\section{Linear Chain Functionalized Gold Nanoparticle in Aqueous Solution}

In this chapter, atomistic molecular dynamics (MD) simulations of linear chain functionalized gold nanoparticle (LCFGNP) with different grafting densities and terminations in aqueous $\mathrm{NaCl}$ solutions are discussed. Special focus has been given to the penetration depth of water and ions into the diffuse shell of the functionalized alkanethiol chains and its dependence on grafting density and functionalization. Also, attention has been given to water orientation and hydrogen bonding. Some parts of this chapter have been taken from the paper "Conformational Equilibria of Organic Adsorbates on Nanostructures in Aqueous Solution: MD Simulations" published in The Journal of Physical Chemistry C, 2015, 119 (45), pp 25566-25575, and are indicated by quotes.

\subsection{Modeling and Method}

Linear functionalized GNPs ${ }^{93-96}$ with gold core (consisting of $144 \mathrm{Au}$ atoms) have been modeled as in Ref. ${ }^{57}$. The gold core is modeled with three shells and possesses nearly spherical geometry. The first gold shell is a polyhedron with 20 faces, 30 edges and 12 vertices, and contains $12 \mathrm{Au}$ atoms. The second shell is also a polyhedron with 80 triangular faces, 120 edges and 42 vertices, and contains $42 \mathrm{Au}$ atoms. The third shell is a rohombicosidodecahedron and it has 20 regular triangle faces, 30 square faces, 12 regular pentagonal, 60 vertices and 120 edges. It contains $60 \mathrm{Au}$ atoms (Figure 4.1). The surface layer of the gold core is covered by 30 "oxidized" Au atoms, each of which is connected to two bridging sulfur atoms $(\mathrm{S}-\mathrm{Au}-\mathrm{S})^{42,97,98}$ (Figure 4.2). The altogether 60 sulfur atoms on the surface of the GNP with an approximate diameter of $2 \mathrm{~nm}$ are then (partially or 


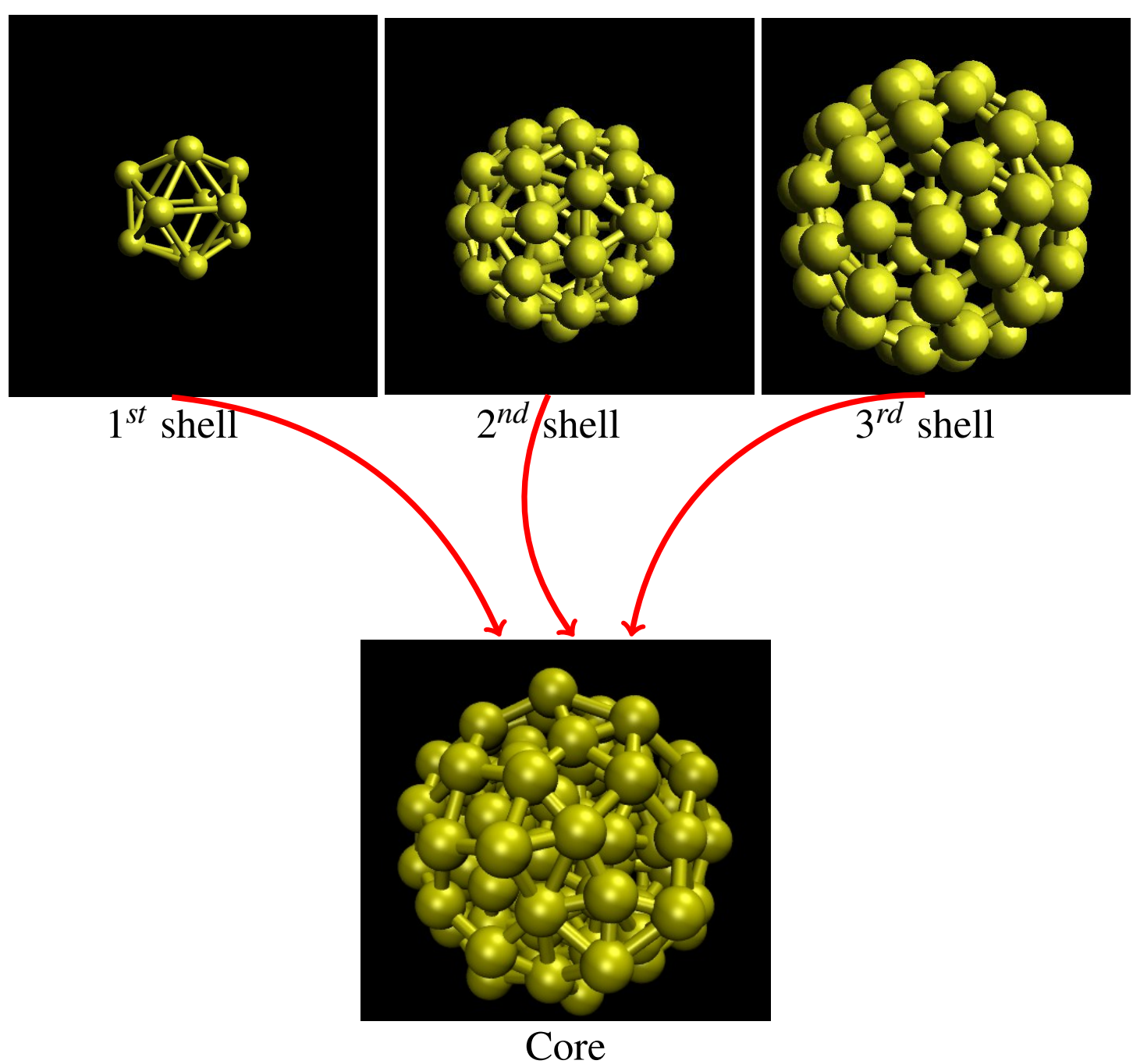

Figure 4.1: Snapshot of the first shell (top-left), the second shell (top-center) and the third shell (top-right) of the gold core (bottom).

fully) functionalized by attaching $N_{C}=20,40$, or $60-\left(\mathrm{CH}_{2}\right)_{11}-\mathrm{CH}_{3},-\left(\mathrm{CH}_{2}\right)_{11}-\mathrm{COO}^{-}$, or $-\left(\mathrm{CH}_{2}\right)_{11}-\mathrm{NH}_{3}^{+}$chains, leading to degrees of functionalization $d_{f}=1 / 3,2 / 3$, and 1 .

A total of 9 systems with coverage varying between $1 / 3$ and 1 and total charge varying between $-60 e$ and $+60 e$ (with $e$ the proton charge) have thus been prepared. All the systems are classified in the following diagram. 


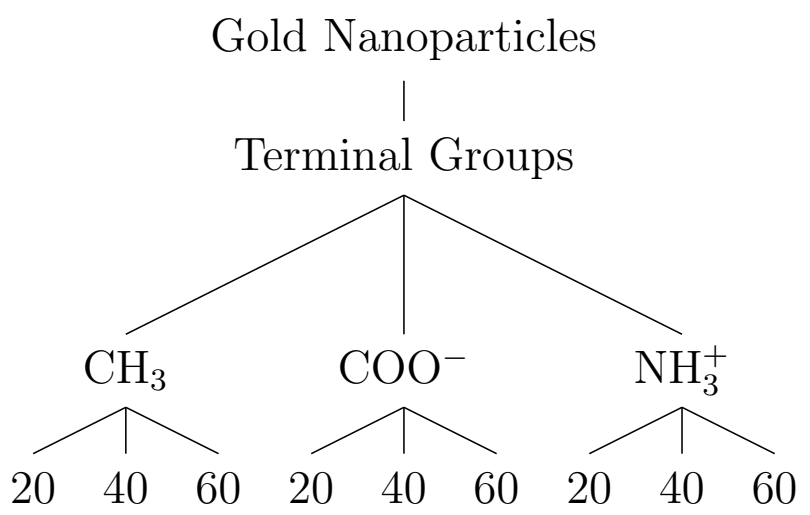

The simulation box size is $8 \times 8 \times 8 \mathrm{~nm}^{3}$ and it contains a single functionalized GNP and approximately 15000 water molecules. Excess $\mathrm{Na}^{+}$and $\mathrm{Cl}^{-}$counter ions are added to the system to compensate the net charge of the GNP and create an overall neutral system (Figure 4.3). In addition, $300 \mathrm{NaCl}$ ion pairs were introduced as background electrolyte, leading to an approximately one molar aqueous solution.

The TIP3P ${ }^{87}$ water model has been used for all simulations together with the CHARMM-27 force field parameters for $\mathrm{Na}^{+}$and $\mathrm{Cl}^{-}$ions ${ }^{86,71}$. Parameters for the functionalized chains have been taken from the AMBER-99 force field ${ }^{84}$, and parameters for gold atoms were taken from Ref. ${ }^{42,99}$, since they were not available in the AMBER-99

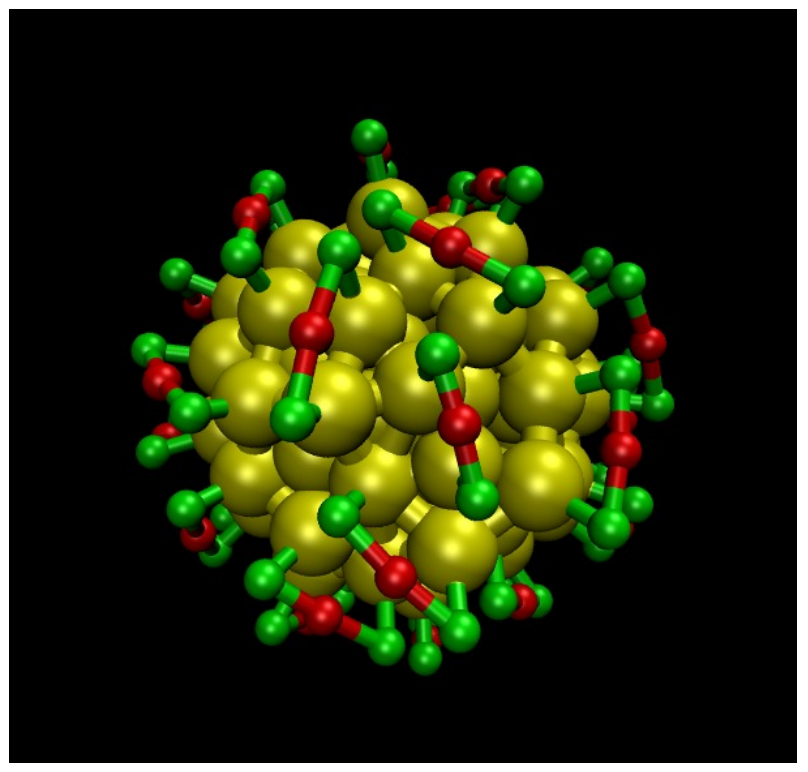

Figure 4.2: Snapshot of surface of the gold core. Green balls are sulfur atoms and red balls are oxidized gold atoms. 
force field. The CHARMM dihedral style ${ }^{100,101}$ has been used for the $\mathrm{C}-\mathrm{C}-\mathrm{C}-\mathrm{C}$ dihedral angles. All parameters are given in Table 4.1.

All simulations are performed with the LAMMPS ${ }^{89}$ simulation package (version 31, March, 2011) using the NVT ensemble, where the number of atoms, volume and temperature of the system are held constant. A Nosé-Hoover thermostat ${ }^{59}$ has been used to keep the temperature of the systems constant at $300 \mathrm{~K}$ with a time constant of 1 ps. The number of water molecules was arranged in preliminary runs to produce a bulk density very close to the experimental density. We ran the simulations at constant volume

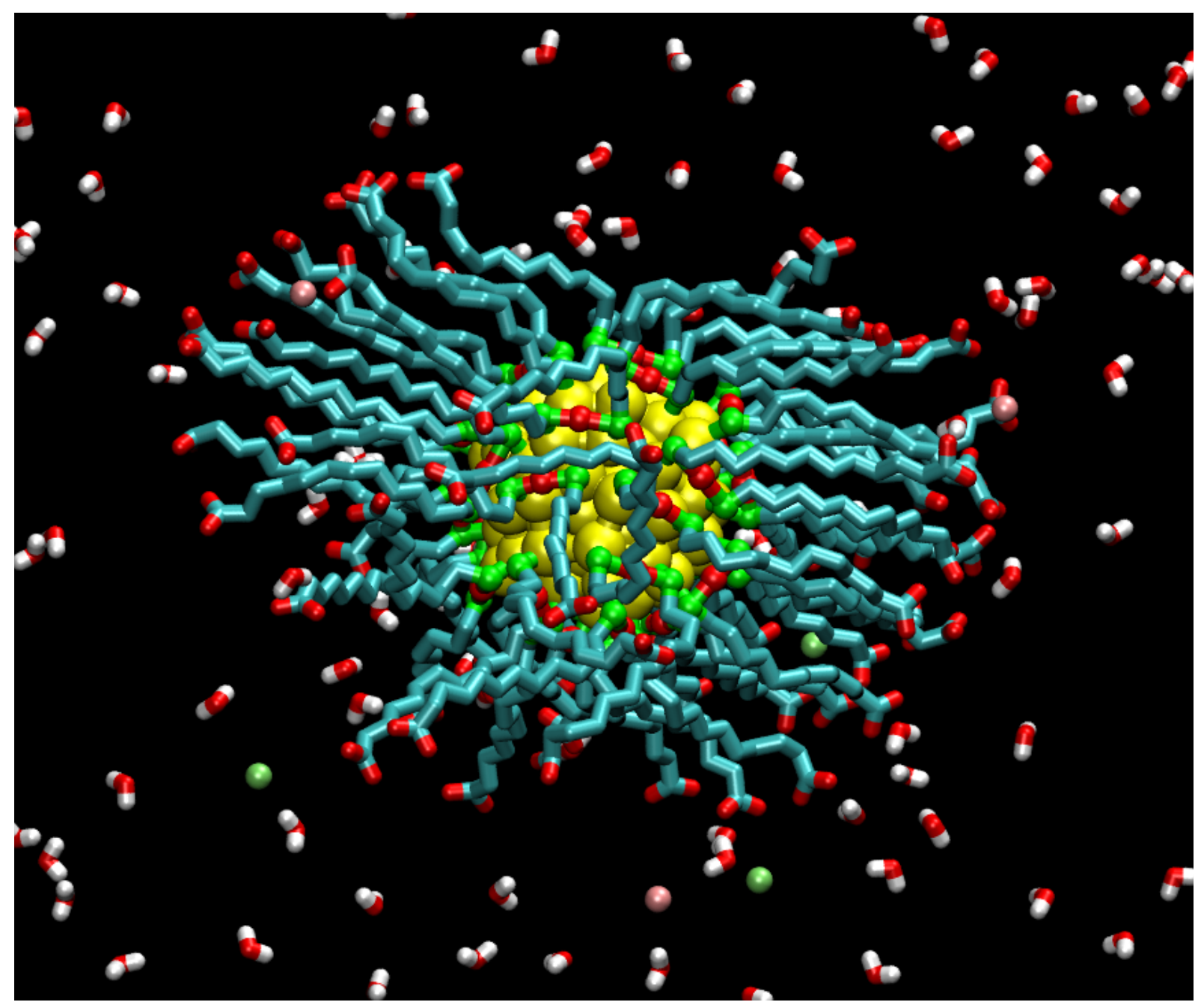

Figure 4.3: Snapshot of a linear chain functionalized gold nanoparticle (LCFGNP) with $60-\left(\mathrm{CH}_{2}\right)_{11} \mathrm{COO}^{-}$chains in solution. Green and pink balls are $\mathrm{Na}^{+}$and $\mathrm{Cl}^{-}$ions, respectively. Only, the ions and water molecules close to the GNP are shown. 
Table 4.1: Force field parameters

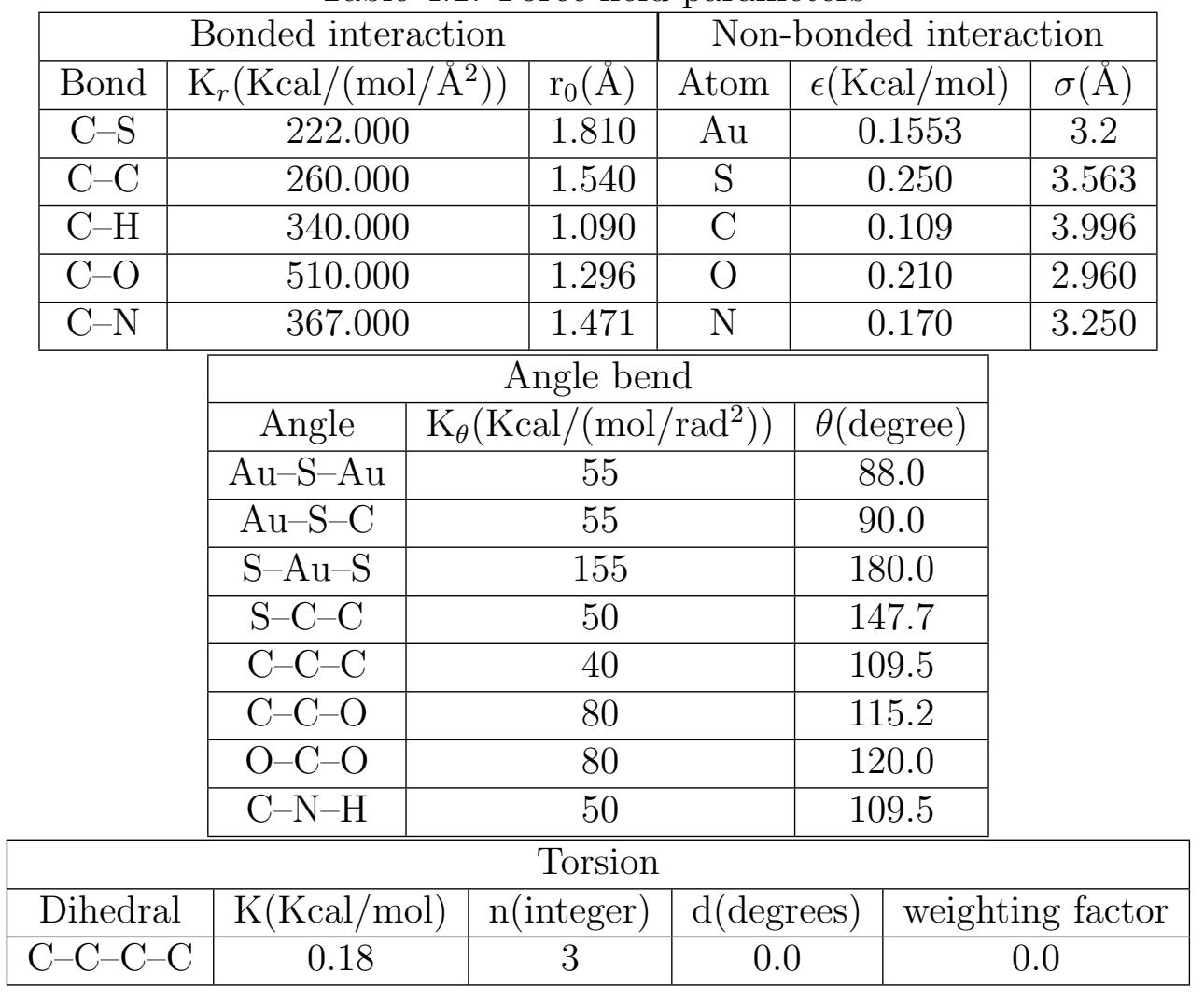

in order to avoid possible instabilities leading to large volume fluctuations due to the very disparate masses of the objects in the simulation cell. Production runs are performed for about 40 ns with a time step of 1 fs and a skin distance for the neighbor tables of $0.1 \mathrm{~nm}$, which were updated every 5 steps. The particle-particle-particle-mesh (PPPM) method to compute long-range Coulomb interactions has been employed with a relative accuracy of $10^{-4}$. The SHAKE algorithm ${ }^{90}$ has been used to constrain O-H bonds and $\mathrm{H}-\mathrm{O}-\mathrm{H}$ angles. The Lennard-Jones interactions are cut off at a distance of $10 \AA$.

The functionalized gold nanoparticles were constructed with the molecular editor Avogadro ${ }^{102}$. $\mathrm{Na}^{+}$and $\mathrm{Cl}^{-}$ions were added with the use of VMD (1.9.1) ${ }^{88}$ employing the TopoTools plugin. Initially, the GNPs were equilibrated in vacuum. Then the box was filled with water and ions. The number of water molecules was adjusted to yield the experimental value of 33 water molecules per $\mathrm{nm}^{3}$ at ambient conditions. Following a 5 ns equilibration run, the dynamics of the GNPs in solution was followed over $40 \mathrm{~ns}$ in production runs. 


\subsection{Results}

\subsubsection{Shape of Gold Nanoparticles}

Figure 4.4 shows snapshots of functionalized GNPs after about 35 ns. The left column shows the snapshots of $\mathrm{CH}_{3}$ group terminated GNPs of density of functionalization $\left(d_{f}\right)=1 / 3$ (top) and 1 (bottom), and the right column shows the snapshots of $\mathrm{COO}^{-}$ group terminated GNPs of $d_{f}=1 / 3$ (top) and 1 (bottom). The snapshots in the top row show that the chains are folded around the gold core, but the snapshots in the bottom

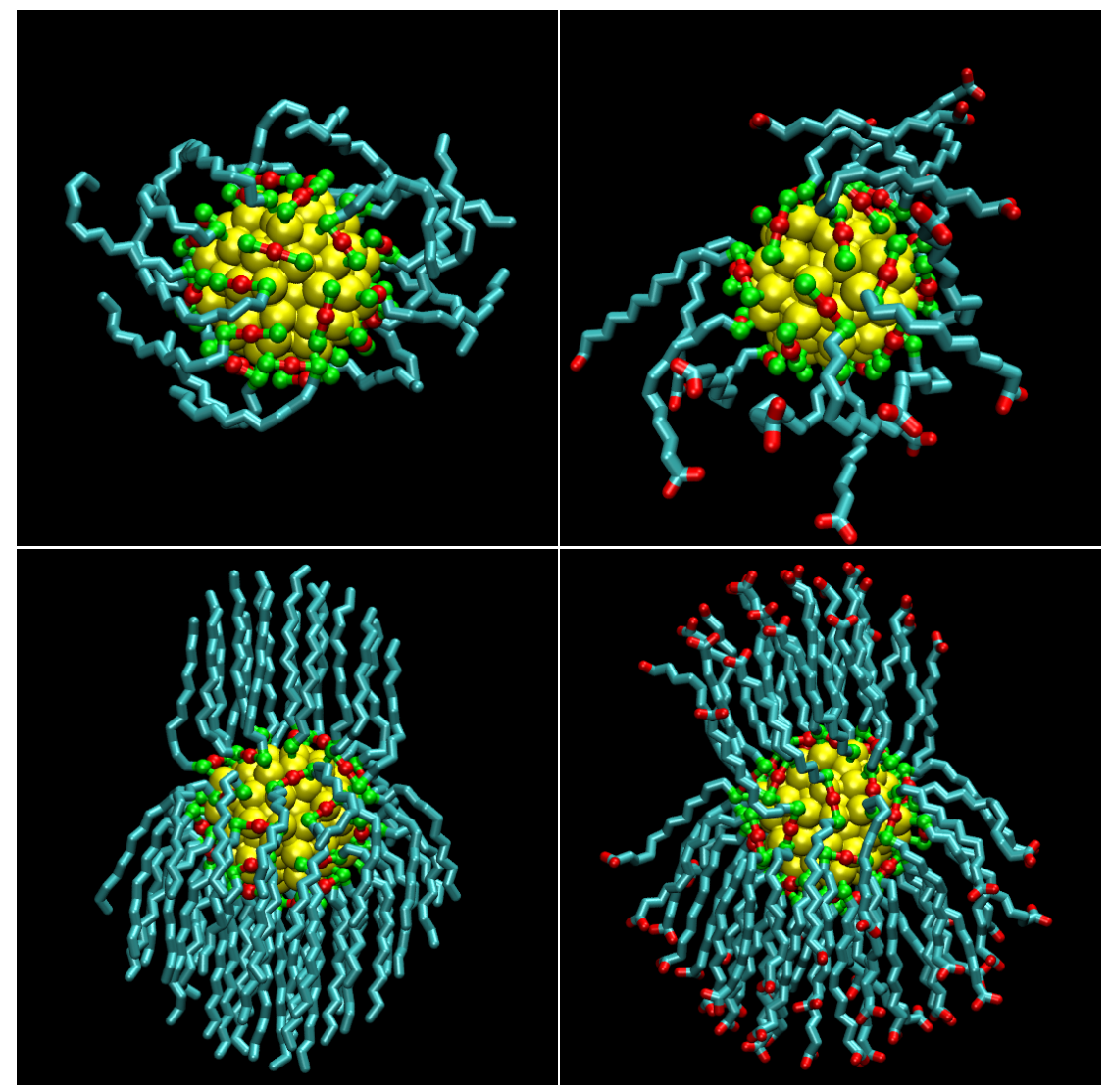

Figure 4.4: Left column: CH3 group terminated GNPs of $d_{f}=1 / 3$ (top) and 1 (bottom). Right column: $\mathrm{COO}^{-}$group terminated GNPs of $d_{f}=1 / 3$ (top) and 1 (bottom).

row show that the chains are predominantly stretched outward irrespective of terminal groups. The snapshots of fully functionalized GNPs show the bunching of chains on the gold surface. This bunching occurs due to multiple effects like size of gold core, graft- 
ing density, inter-chain interactions, and interactions between solution and chains. The bunching of chains is more ordered in case of nonpolar chains than for the polar chains, because the nonpolar chains do not have any repulsive electrostatic interactions between the chains. The bunching of chains leads to coating asymmetry on gold cluster, although the chains are grafted isotropically on the gold core. This phenomenon is not a computational artifact. Evidence for such asymmetry of isotropically functionalized nanoparticle was recently observed on the basis of light scattering experiments. ${ }^{103}$ At low coverage, the nonpolar chains fall back on the gold core to reduce solvent accessible surface area (for more details see chapter 5). Thus a GNP forms, which is covered by disordered hydrocarbon-chains. At high coverage, the chains stretch out mainly due to the crowded environment close to the GNP, where not much room is available for gauche defects of individual chains. At larger distance from the surface, enough room is available for the chains to fall back over the gold surface but the chains form bundles to reduce the solvent accessible surface area.
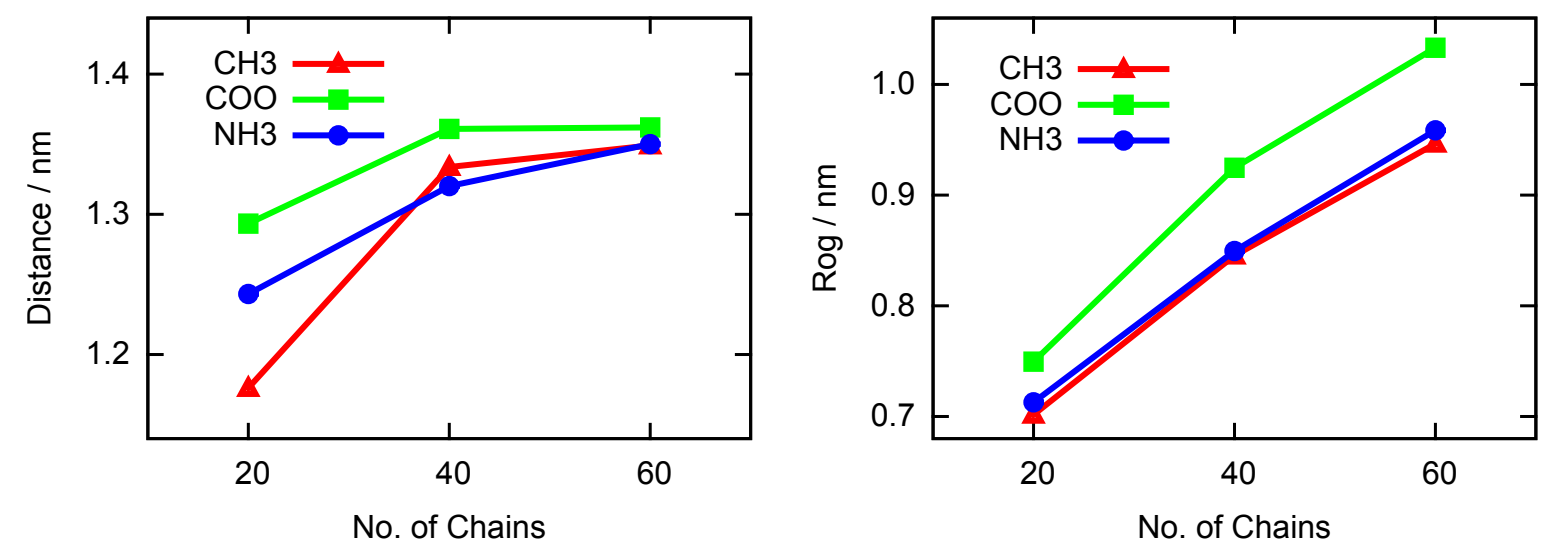

Figure 4.5: The left side shows the time averaged chain lengths of hydrocarbon chains on gold cores. The right side shows the radius of gyration of all systems.

In figure 4.5 on the left side, time averaged chain lengths are compared for different systems. The chain lengths are measured as the distance between the first carbon (connected to sulfur) and the methyl carbon or the carboxylic carbon or the amine nitrogen of the same chain, and averaged over time. GNPs with high grafting densities (40 and 60 chains) have nearly equal chain length, but the difference of chain length between 40 and 20 chains GNPs is rather high. All 60 chain GNPs have more or less the same chain lengths irrespective of their terminal groups. $\mathrm{COO}^{-}$terminated chains are always slightly longer than the $\mathrm{CH}_{3}$ and the $\mathrm{NH}_{3}^{+}$terminated chains. Differences between chain lengths 
of different terminal groups increase with decreasing grafting density. The chain length of $\mathrm{CH}_{3}$ terminated GNPs is shorter than that of polar group terminated chains at low grafting density (20 chain GNPs). In figure 4.5 on the right side, the radius of gyration $\left(\mathrm{R}_{g}\right)$ of the systems are plotted. The radius of gyrations are calculated taking all atoms of a functionalized GNP. The radii of gyration of $\mathrm{COO}^{-}$terminated systems are always

high, irrespective of grafting density. $\mathrm{R}_{g}$ of $\mathrm{CH}_{3}$ and $\mathrm{NH}_{3}^{+}$terminated systems are similar, and the radius of gyration of the systems validates the results of chain length.

High grafting density on the gold core and bundle formation of chains leads to the extended and nearly equal chain length. At low density, the chains collapse on the gold core reducing the chain length. Polar group terminated chains at low density have much longer chain length than the non-polar group terminated chains due to the Coulomb interaction between polar terminals. Water and ion structure around the $\mathrm{COO}^{-}$terminal group leads the chains to stretch out more than for the other terminations. The high weight of $\mathrm{COO}^{-}$terminal groups and the stretched chains help to have higher $\mathrm{R}_{g}$ values of $\mathrm{COO}^{-}$terminated systems than the other groups terminated systems.

\subsubsection{Penetration of Water and Ions}

"Figure 4.6 shows radial density $\left(R_{d}\right)$ distribution of various atoms and ions of different systems. At low coverage $\left(d_{f}=1 / 3\right.$, left column), the distributions of chain atoms (red lines) are closer to the GNP (chain atoms belong in the region $1 \mathrm{~nm}$ to $2 \mathrm{~nm}$ from the center of the gold core) than for the higher chain coverage. The chain atoms of the $\mathrm{CH}_{3}$ group terminated chains at $d_{f}=1 / 3$ (left top) have their maximum density at the same distance (1.2 nm from the center of the gold core), and the terminal chain atoms $\left(\mathrm{C}_{\text {term }}\right)$ display a wider and more asymmetric distributions than the other chain atoms. These distributions of chain atoms indicates that the chains wind around the gold surface. The maxima of the distributions of polar group $\left(\mathrm{COO}^{-}\right.$or $\left.\mathrm{NH}_{3}^{+}\right)$terminated chain atoms shift away from the gold surface when moving from atom $\mathrm{C}_{6}$ towards the end of the chain. Also, the width of the distributions of the chain atoms near the polar terminal groups are much wider than in the left-top figure. These distributions indicate that the thickness of the hydrocarbon shells is larger for the polar group terminated GNPs than for the nonpolar group terminated GNP. Also, the distributions indicate that there is a competition between pulling the individual chains to the surface and solvating the polar terminal groups in a more stable manner further away from the surface. 


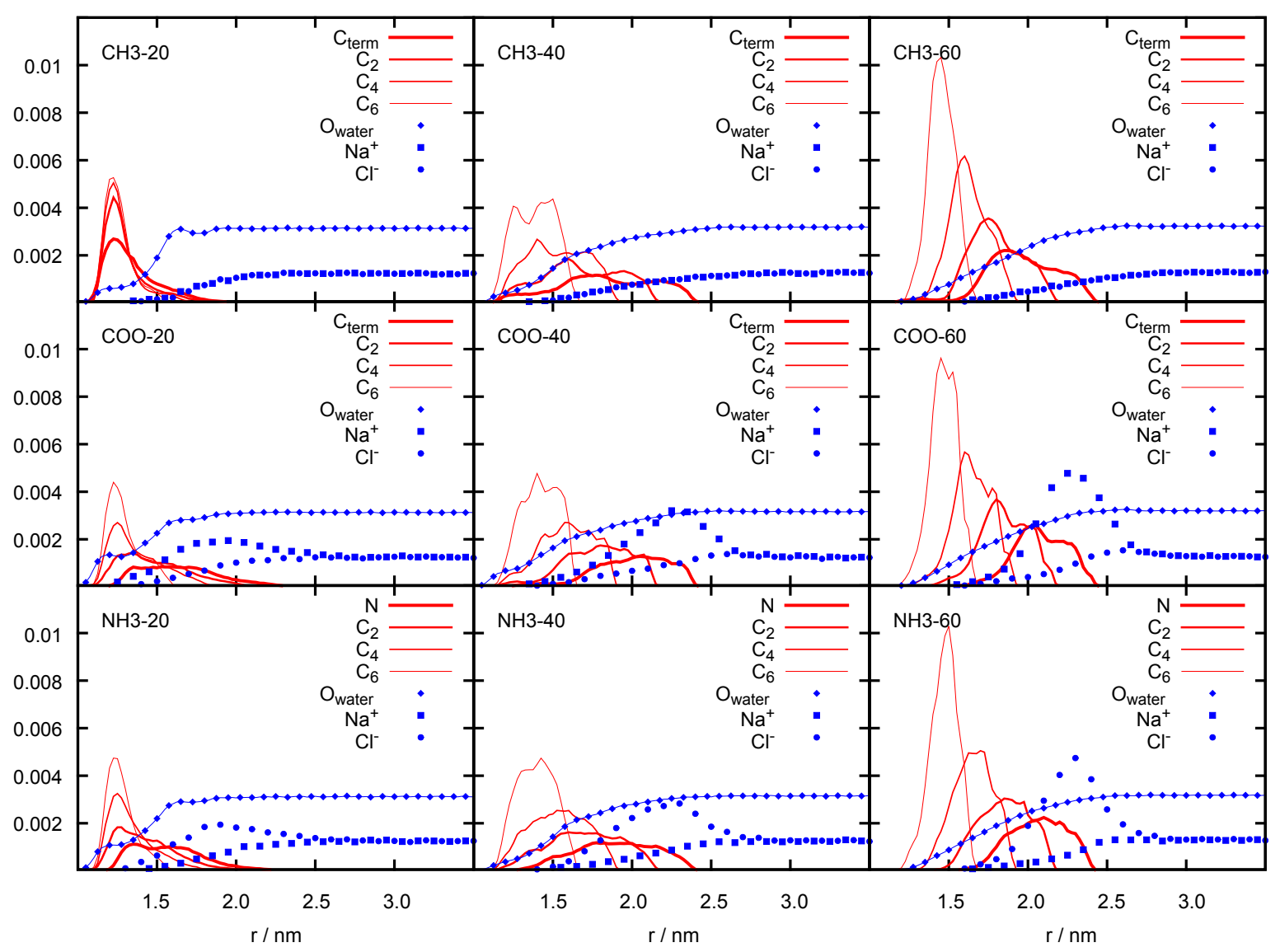

Figure 4.6: Radial density distribution around the gold core for the functionalized GNPs of $d_{f}=1 / 3$ (left column), $2 / 3$ (central column) and 1 (right column). The top row, the central row and the bottom row show the distribution for $\mathrm{CH}_{3}, \mathrm{COO}^{-}$and $\mathrm{NH}_{3}^{+}$ group terminated GNPs, respectively. Red lines of varying thickness show the density distribution corresponding to chain atoms. The widest red lines are corresponding to terminal atoms $\left(\mathrm{C}\right.$ of $\mathrm{CH}_{3}$ or $\mathrm{C}$ of $\mathrm{COO}^{-}$or $\mathrm{N}$ of $\left.\mathrm{NH}_{3}^{+}\right)$and other narrower red lines are corresponding to 2nd, 4th and 6th (narrowest line) carbon atoms of a chain (counting started from the terminal one towards the gold surface). The density distributions of $\mathrm{Na}^{+}, \mathrm{Cl}^{-}$and water oxygen are shown in blue-squares, blue-circles and blue-diamonds, respectively. The vertical scales have been adjusted to bring all densities in same scale using identical scale factors for all red lines, one for ions, and one for water oxygen.

When degree of functionalization is increased from $1 / 3$ to $2 / 3$ and above, the special constraints for the densely packed chains become more pronounced. This leads to a progression of the density maxima of chain atoms away from the surface when moving from atom $\mathrm{C}_{6}$ towards the end of the chain. The progression of maxima is more regular at 
$d_{f}=1$ (right column) than at $d_{f}=2 / 3$ (central column). The width of the distributions of chain atoms is wider at $d_{f}=2 / 3$ (central column) than at $d_{f}=1 / 3$ (left column), and this is true irrespective of the terminal group. Also, the width becomes wider, the further the chain atom is located away from the sulfur pivot point. The room for the conformational freedom of the chains at $d_{f}=2 / 3$ is quite large, whereas at $d_{f}=1$ only the outer parts of the chains have enough room for gauche defects.

The distribution of the oxygen atoms of the water molecules at $d_{f}=1 / 3$ shows that the number of water molecule close to the gold surface is almost half for nonpolar group terminated GNP system (left top) compared to the polar group terminated GNP systems (left center and left bottom). This is visible as a step in the density at around $1.2 \mathrm{~nm}$. Another structural feature is visible at around $1.7 \mathrm{~nm}$, where water oxygen density is depressed slightly. At high grafting densities $\left(d_{f}=2 / 3\right.$ and 1$)$, the step feature of the water density is invisible and the densities increase more or less monotonically from zero value towards the bulk density at a distance of around $2.4 \mathrm{~nm}$ from center of the gold core. In all cases, few water molecules penetrate the chain regions and reach very close to the gold surface, and the number of such molecules decreases with increasing grafting density and polarity. Ions can not reach close to the gold surface like water, and the penetration of ions decreases with increasing grafting density. For nonpolar group terminated GNP systems (top row) ion density increase monotonically from zero to a bulk value irrespective of different chain coverage. For polar group terminated GNPs, counter ion densities do not increase monotonically; rather counter ions accumulate near the polar terminal groups, and that produces a high counter ion density region near the polar terminal groups. This accumulation of ions near the polar terminal groups increases with increasing polarity.

Figure 4.7 shows color coded maps of the distribution of the closest atoms (chain carbon or oxygen of water) to the gold surface. The left column shows color coded maps for $\mathrm{CH}_{3}$ group terminated GNPs of varying coverage $d_{f}=1 / 3,2 / 3$ and 1 (top to bottom). The right column shows color coded maps for $\mathrm{COO}^{-}$group terminated GNPs of varying coverage $d_{f}=1 / 3,2 / 3$ and 1 (top to bottom). Along the vertical direction the cosine of the polar angle $\theta$ varies between +1 and -1 , and along the horizontal direction the azimuthal angle $\phi$ varies between 0 and $360^{\circ}$. In the color maps, the largest values (1, yellow) indicate that oxygen atoms of water molecules are very close to the gold surface and purple color indicates that carbon atoms of chains are close to the surface. The variation of colors in the maps is partly due to the limited sampling time of $40 \mathrm{~ns}$, but this variation of color is mostly a consequence of the anisotropic distribution of chain 


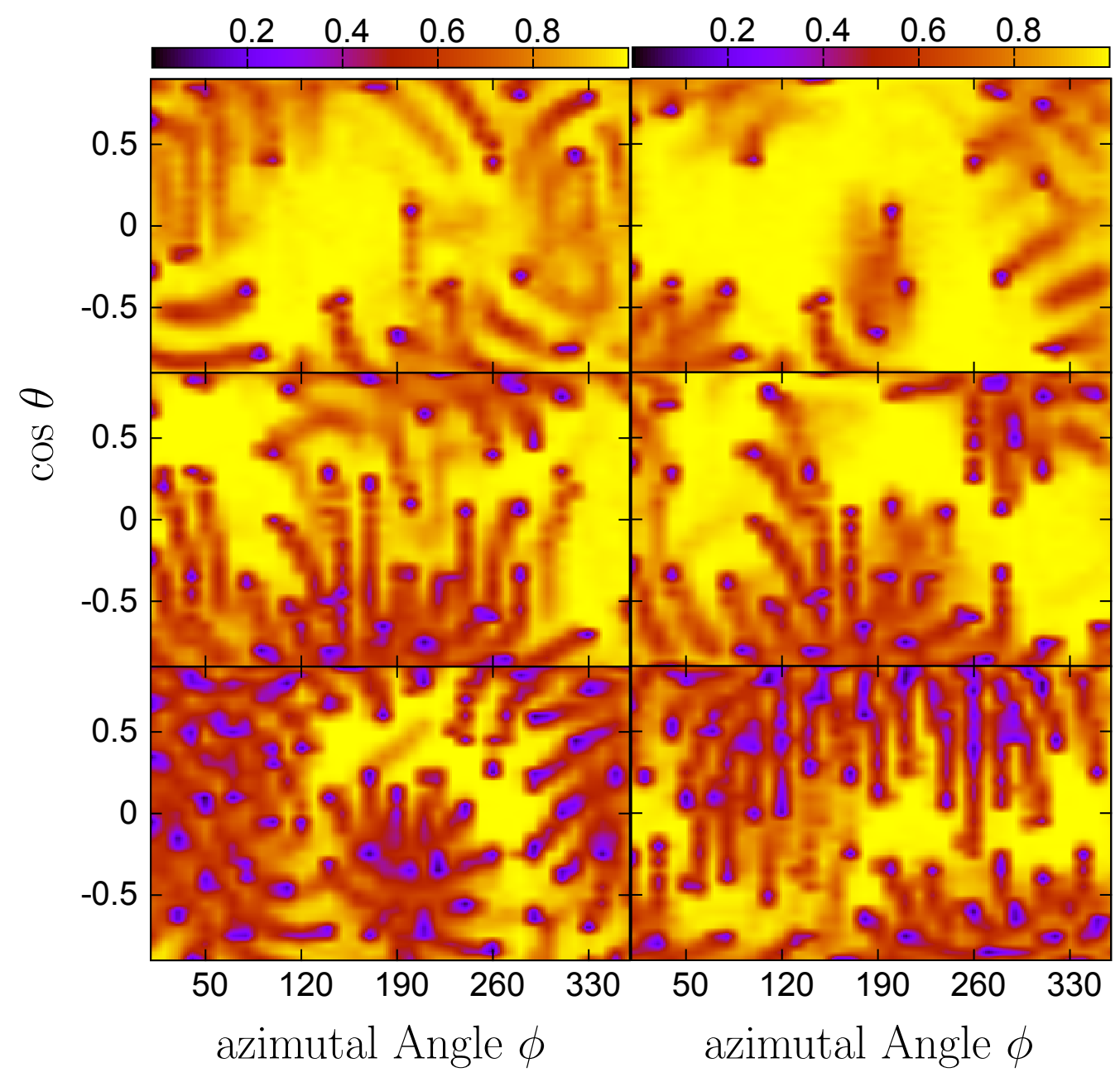

Figure 4.7: Color maps of surface access. Left column: $\mathrm{CH}_{3}$ group terminated chains with $d_{f}=1 / 3$ (top), $2 / 3$ (center) and 1 (bottom). Right column: $\mathrm{COO}^{-}$group terminated chains with $d_{f}=1 / 3$ (top), $2 / 3$ (center) and 1 (bottom). Values 1 (yellow) indicate that a water molecule is closest to the GNP surface, while a value 0 indicates that a chain atom is closest to the surface. All the maps show entire surface of the unit sphere with azimuthal angle $\phi$ between 0 and $360^{\circ}$ and the cosine of the polar angle theta between -1 and +1 .

atoms.

The top two maps show that water can access the gold surface very easily. It seems that the yellow regions for the polar group $\left(\mathrm{COO}^{-}\right)$terminated GNP is larger than for the nonpolar group $\left(\mathrm{CH}_{3}\right)$ terminated GNP. This indicates that the chains of polar group terminated GNP are more stretched out than the nonpolar group terminated GNP, and the chains of nonpolar group terminated GNP cover the gold core better than the chains 
of polar group terminated GNP. As the grafting density increases (from top to bottom) the yellow regions become smaller and smaller, which means that the water access to the gold surface decreases with increasing coverage. However, even at the highest coverage $\left(d_{f}=1\right)$ chains can not protect the gold core from water, and water can permanently access the gold surface. In summary these plots show that water can access the gold surface of all studied GNPs irrespective of their termination on a time scale of $40 \mathrm{~ns}$ and beyond.

Corresponding plots of surface access of the remaining systems (given in appendix A.2) show features similar to the ones discussed here. It is interesting to note that the domain formation of the chain 'bundles' is statistical in the sense that each simulation shows a different arrangement of water-accessible and chain-covered surface regions.

The left column of figure 4.8 shows the distribution of coordination numbers for $\mathrm{Na}^{+}$ions around the $\mathrm{COO}^{-}$group terminated GNPs. The bold frames (from top to bottom) represent the coordination number distributions with increasing degrees of functionalization between $1 / 3$ and 1 . A single bold frame represents the coordination number distributions separately for water coordination, $\mathrm{COO}^{-}$coordination and total coordination (from top to bottom). Different coordinations are calculated by counting the total number of oxygen of water molecules, total number of terminal oxygen of $\mathrm{COO}^{-}$groups, and total number of terminal oxygen and water oxygen together within a distance of 0.32 $\mathrm{nm}$ from the $\mathrm{Na}^{+}$ions. Using this definition, the most probable total coordination number far away from the GNP is 5 .

The $\mathrm{Na}^{+}$ions loose part of their water solvation shell when they penetrate in the hydrocarbon chain regions and this is true for all values of $d_{f}$. However, this loss can be compensated by the terminal oxygens in terminal group region ( $\approx 1.8$ to $2.7 \mathrm{~nm}$ ). In the region between 2 to $2.3 \mathrm{~nm}$ from the center of the gold core and the bulk, the distribution of total coordination number does not vary with distance and the terminal group oxygens replace the water molecules from ion solvation shells. At short distance from the gold surface the terminal oxygens can not compensate the loss of water solvation and thus the total coordination number for $\mathrm{Na}^{+}$ions shifts towards smaller values.

The right column of figure 4.8 shows the distribution of coordination numbers for $\mathrm{Cl}^{-}$ions around the $\mathrm{NH}_{3}^{+}$group terminated GNPs, which is analogous to the distribution of coordination numbers for $\mathrm{Na}^{+}$ions. The bold frames (from top to bottom) represent the distribution of coordination numbers with increasing degrees of functionalization between $1 / 3$ and 1 like in left column. A single bold frame represents the coordination number 

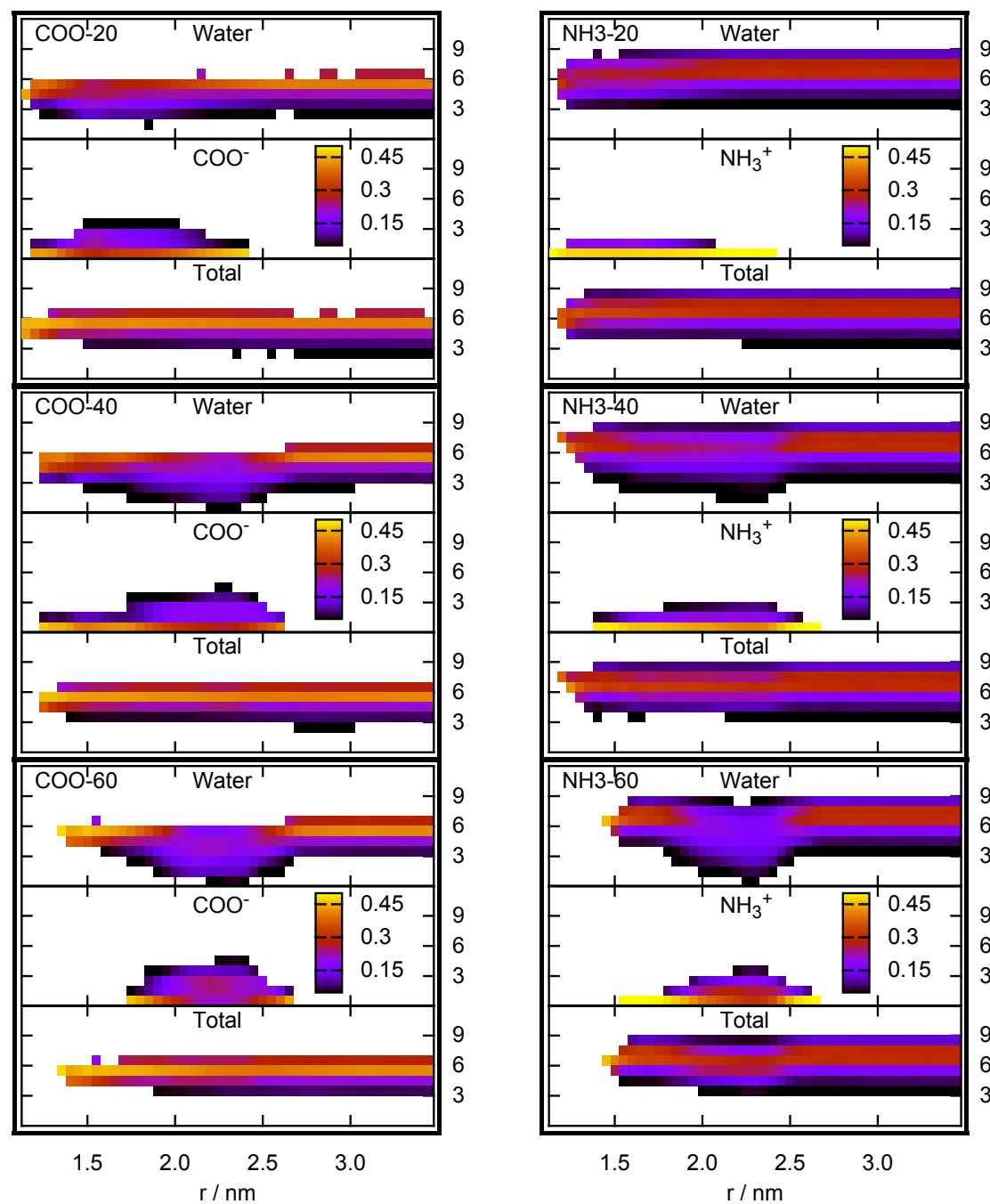

Figure 4.8: Solvation number distributions for ions for different systems. Left Column: solvation number distribution for $\mathrm{COO}^{-}$group terminated GNP systems. The color code shows the probability to find given coordination numbers (vertical axis) around $\mathrm{Na}^{+}$ions in dependence of the distance of the ion from the GNP center. Each bold frame shows coordinated water, coordinated $\mathrm{COO}^{-}$oxygen atoms and total coordination number. The bold frames from top to bottom are for increasing degrees of functionalization between $1 / 3$ and 1 . Coordination numbers are calculated counting number of oxygen atoms inside a sphere of radius $0.32 \mathrm{~nm}$ around the $\mathrm{Na}^{+}$ions. Right Column: analogous solvation number distribution for $\mathrm{Cl}^{-}$ions for $\mathrm{NH}_{3}^{+}$group terminated GNP systems. For this case coordination numbers are calculated counting number of hydrogen atoms within a sphere of radius 0.29 around $\mathrm{Cl}^{-}$ions. 
distributions separately for water coordination, $\mathrm{NH}_{3}^{+}$coordination and total coordination (from top to bottom). Different coordinations are calculated by counting the total number of hydrogens of water molecules, total number of terminal hydrogens of $\mathrm{NH}_{3}^{+}$groups, and total number of terminal hydrogen and water hydrogen together within a distance of $0.29 \mathrm{~nm}$ from the $\mathrm{Cl}^{-}$ions. Using this definition, the most probable total coordination number is 7 or 8 . In the region between 2 and $2.5 \mathrm{~nm}, \mathrm{NH}_{3}^{+}$terminal groups replace almost completely some solvation water molecules. At short distance from the GNP surface, the loss of solvation shell is not compensated and the coordination number distribution shifts towards smaller values. However, the partial loss of solvation shell for the $\mathrm{Cl}^{-}$ions is less than for the $\mathrm{Na}^{+}$ions. Also, the total coordination number distribution for the $\mathrm{Cl}^{-}$ions is less affected than for the $\mathrm{Na}^{+}$ions at short distance from the gold surface.

\subsubsection{Conformation of Hydrocarbon Chains}

Figure 4.9 shows the simulation averaged percentage of trans conformations of the individual dihedral angles along the chain. The trans conformation is defined by considering dihedral angles $\phi$ between 120 and $240^{\circ}$. Average dihedral angles have been calculated separately for each individual chain. Dihedral angle 1 in the figure denotes the angle corresponding to the dihedral which starts from the terminal group $\left(\mathrm{CH}_{3}, \mathrm{COO}^{-}\right.$or $\left.\mathrm{NH}_{3}^{+}\right)$ towards the GNP surface. Dihedral angle 2 denotes the angle corresponding to the dihedral which starts from the second atom from the terminal towards the GNP surface, dihedral angle 3 corresponds to the dihedral which starts from the third atom, and other dihedral angles follow same trend. The error bars in the figure show standard deviations from the mean value and they provide information about the static heterogeneity of the chains.

The top frame of the figure shows the simulation averaged percentage of trans conformations for polar group $\left(\mathrm{NH}_{3}^{+}\right)$terminated chains for different degrees of functionalization. The first dihedral angle shows less trans and more gauche conformation than the second to fifth dihedral angles. Dihedral angles close to the gold surface again show less trans and more gauche conformation. Overall the trans conformation of angles increases with increasing grafting density because the increased crowding near the gold surface prohibits the gauche conformation which needs more space than the trans conformation. However, the static heterogeneity increases (see the error bars) with increasing dihedral angle number (except first dihedral angle) and grafting density. 


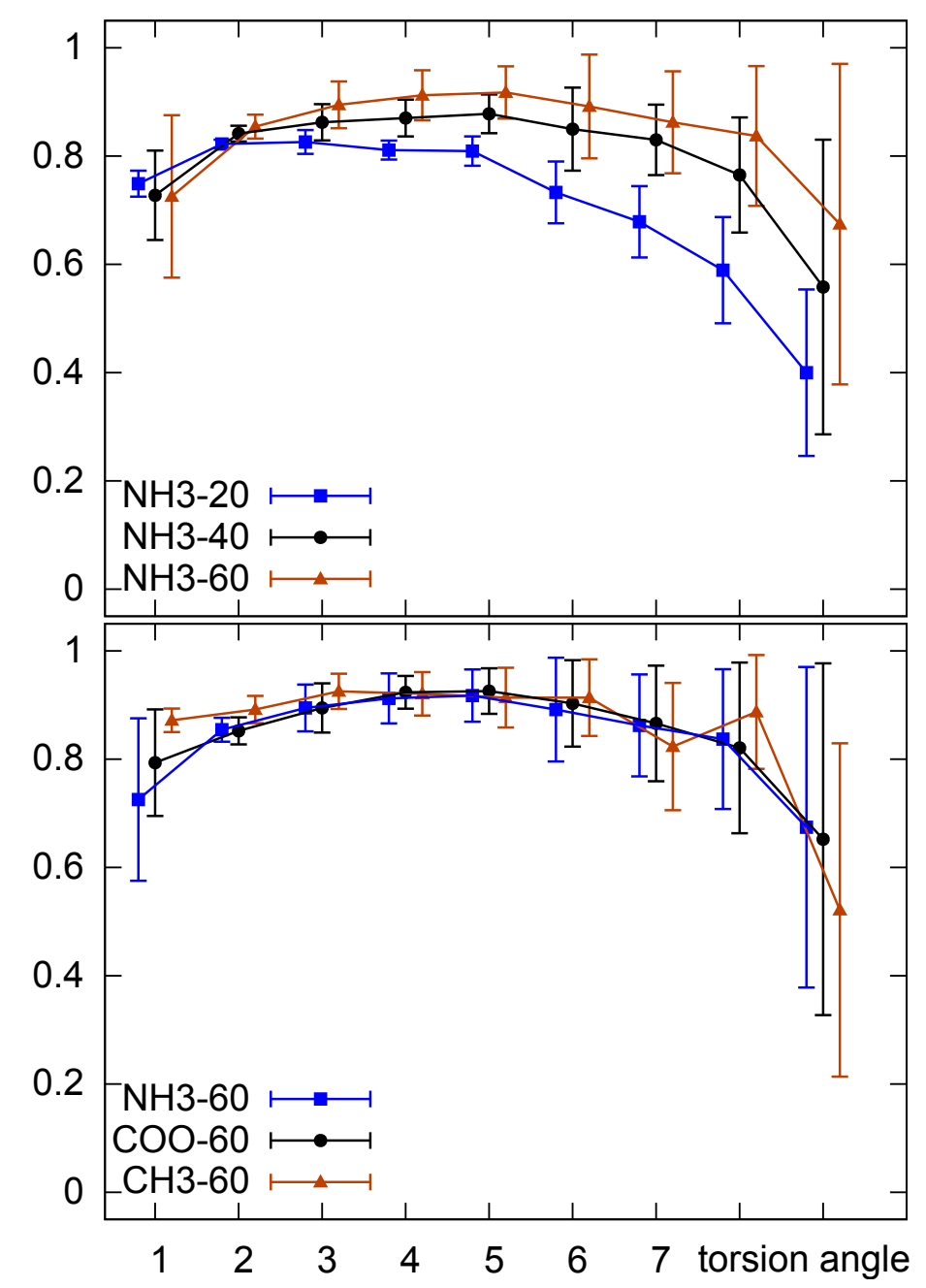

Figure 4.9: Fraction of trans conformations for individual dihedral angles. Top frame is for $\mathrm{NH}_{3}^{+}$group terminated GNP systems and bottom frame is for fully functionalized GNP systems. The numbering of dihedrals starts from the terminal dihedral (1st dihedral) which contains one heavy atom from the terminal group $\left(\mathrm{C}\right.$ of $\mathrm{CH}_{3}$ or $\mathrm{C}$ of $\mathrm{COO}^{-}$or $\mathrm{N}$ of $\left.\mathrm{NH}_{3}^{+}\right)$.

The bottom frame of the figure shows the simulation averaged percentage of trans conformations at $d_{f}=1$ for three different functionalizations. The overall trends of trans conformation and static heterogeneity are similar for all functionalizations. However the trans conformation and static heterogeneity of the first dihedral angle for the $\mathrm{CH}_{3}$ group terminated chain is smaller than for the corresponding polar group $\left(\mathrm{COO}^{-}\right.$and $\left.\mathrm{NH}_{3}^{+}\right)$ 
terminated chains.

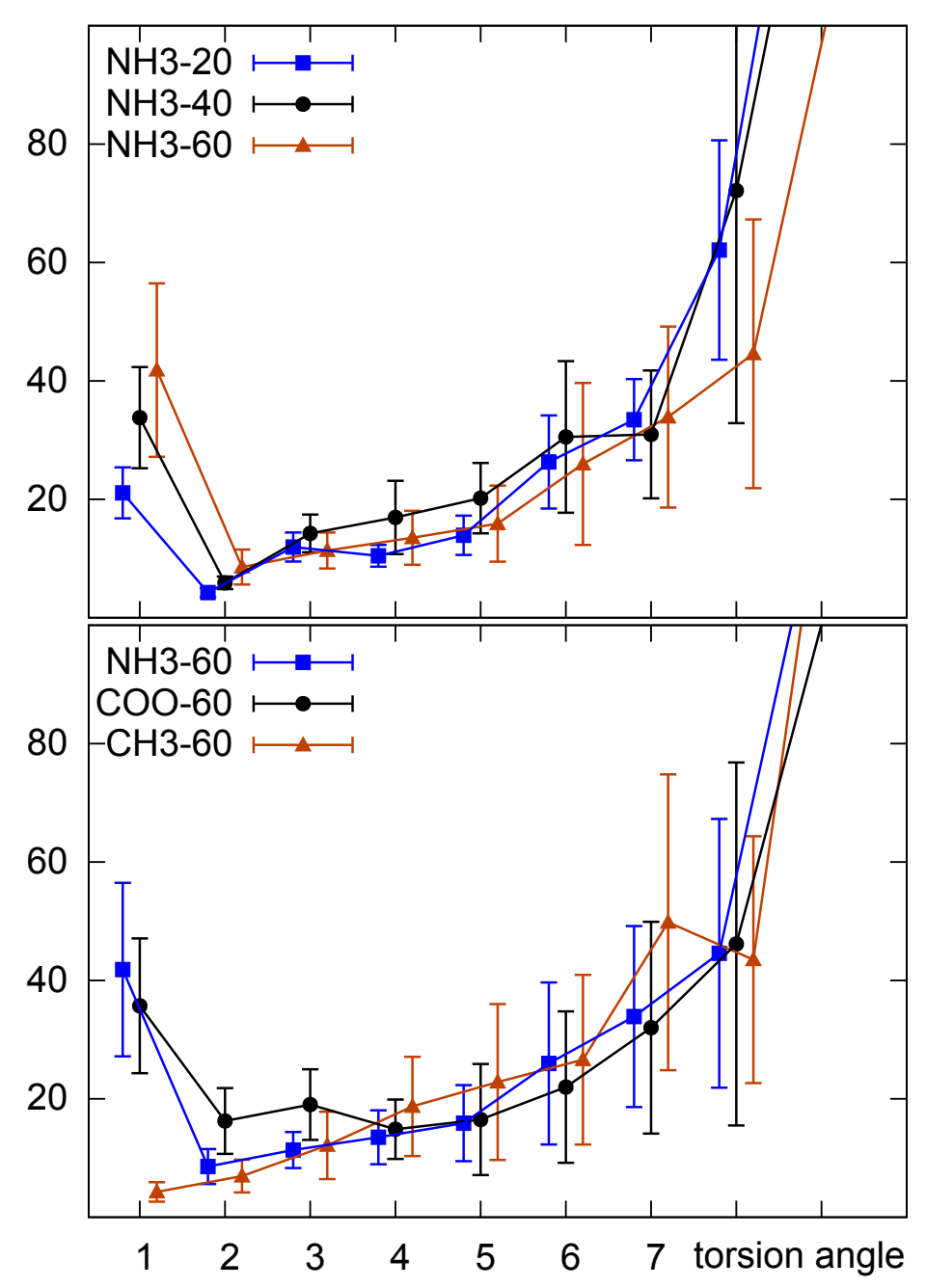

Figure 4.10: Relaxation time of individual torsional angles. Top frame is for $\mathrm{NH}_{3}^{+}$group terminated GNP systems and bottom frame is for fully functionalized GNP systems. The numbering of dihedrals starts from the terminal dihedral (1st dihedral) which contains one heavy atom from the terminal group $\left(\mathrm{C}\right.$ of $\mathrm{CH}_{3}$ or $\mathrm{C}$ of $\mathrm{COO}^{-}$or $\mathrm{N}$ of $\left.\mathrm{NH}_{3}^{+}\right)$. See text for the procedure to calculate the relaxation times.

Fig. 4.7 and fig. 4.9 gave a static view of bundle structure and chain conformation, now fig. 4.10 provides a dynamic view of intra chain dynamics. Figure 4.10 shows the relaxation time of dihedral conformations. The relaxation times of dihedral conformations are calculated individually for each dihedral angles defined in fig 4.9. The error bars in 
the figure shows the standard deviations from the mean value of relaxation time and these standard deviations provide information about the dynamic heterogeneity.

The relaxation time of the dihedral angles is calculated in the following way: dihedrals are categorized into three classes depending on the dihedral angles $\left(\left[0-120^{\circ}\right]\right.$, $\left[120-240^{\circ}\right]$ (trans), and $\left.\left[240-360^{\circ}\right]\right)$. For each class in turn, then the normalized autocorrelation function (acf) is

$$
f_{d i h}=\left\langle h_{c}(0) \cdot h_{c}(t)\right\rangle
$$

with $h_{c}(t)=p_{c}\left(t \mid p_{c}(0)=1\right)-p_{c}(\infty)$. Where $p_{c}\left(t \mid p_{c}(0)=1\right)$ is the probability of a specific dihedral to be in the class $c$ at time t, provided it was in the class $c$ at time $t=0, p_{c}(\infty)$ is the (static) equilibrium probability of finding a specific dihedral in class $c$, and $h_{c}(0)=1$.

The top frame of the figure 4.10 shows the relaxation times for the $\mathrm{NH}_{3}^{+}$group terminated systems of different degrees of functionalization. The terminal dihedrals show sufficiently large relaxation times with respect to the neighboring dihedrals. Also the dynamic heterogeneity increases with increasing grafting density. The relaxation times of the dihedrals close to the gold surface increase drastically which is obviously due to the interaction between chains and physical constraints (such as: large curvature, and bonds between gold cluster and chains).

The bottom frame of the figure shows relaxation times for the fully functionalized GNPs of different functionalization. The overall trend is similar for all the chain terminations. However, the relaxation times of the terminal dihedrals depend on the polarity of the terminal. The relaxation time of first dihedral of nonpolar chain is much lower than the polar ones. Also, the dynamic heterogeneity of the first dihedral of the nonpolar chain is much lower than that of the polar one. The high relaxation time and dynamic heterogeneity of the first dihedrals of polar chains are due to the strong electrostatic interaction between terminal groups, and the water dipoles and counter ions.

\subsubsection{Mobility of Ions and Hydrogen bonds}

Figure 4.11 shows self diffusion coefficients (SDCs) of $\mathrm{Na}^{+}$and $\mathrm{Cl}^{-}$ions. SDCs are calculated from the final values of mean square displacement (MSD) after 0.8 ns. Initially, ions are selected in a spherical shell (radii of the spheres are given along horizontal axis) 

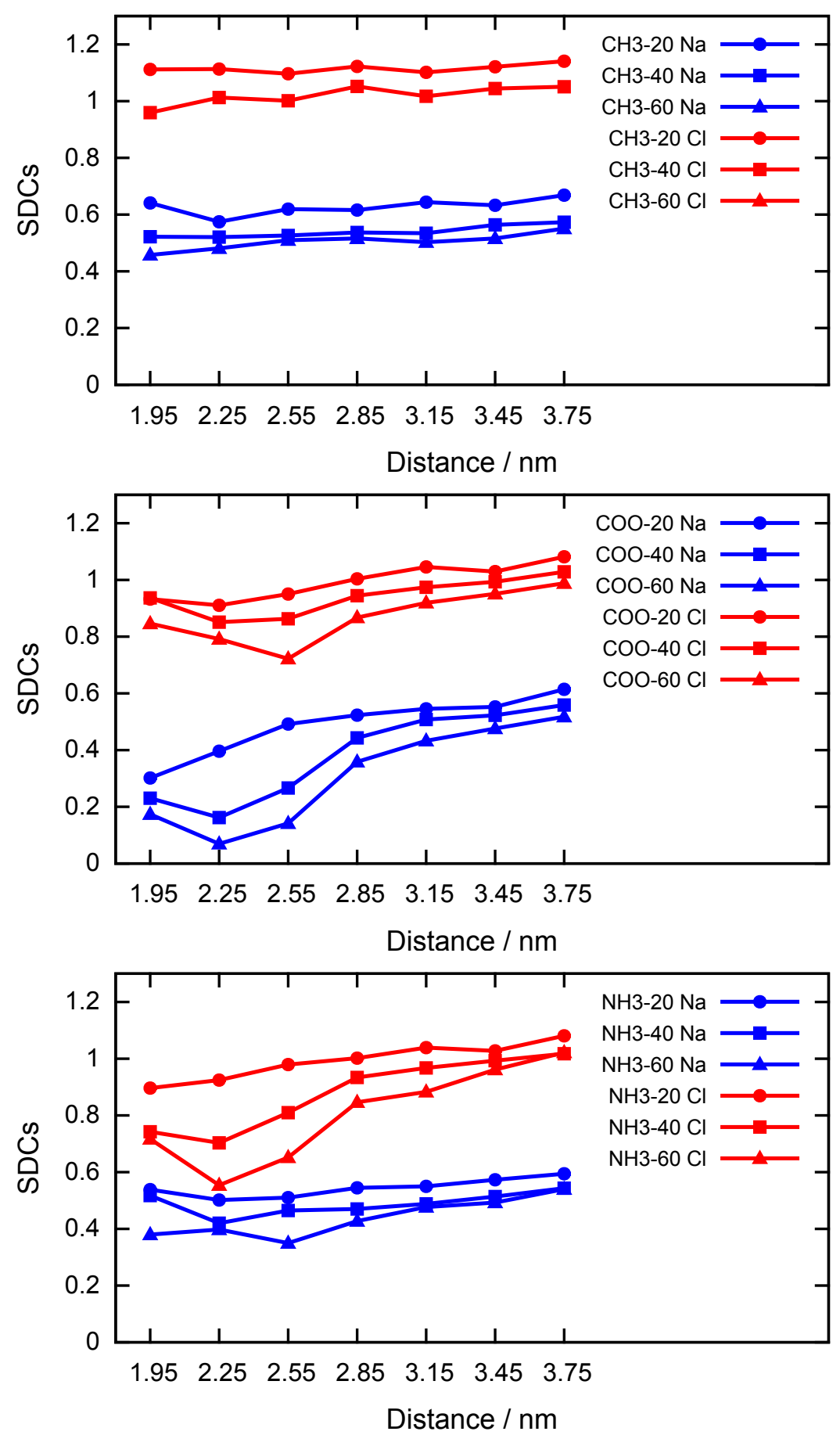

Figure 4.11: Ion self diffusion coefficients (in units of $10^{-5} \mathrm{~cm}^{2} \mathrm{~s}^{-1}$ ). Top, center and bottom frames are for $\mathrm{CH}_{3}$ termination, $\mathrm{COO}^{-}$termination and $\mathrm{NH}_{3}^{+}$termination, respectively. For better visibility, values are shifted upwards by 0.15 units for the $\mathrm{Na}^{+}$ions and downwards by 0.15 units for the $\mathrm{Cl}^{-}$ions. 
of thickness $0.3 \mathrm{~nm}$ and then MSDs are calculated for those ions. For better visibility of the lines, the data for the $\mathrm{Na}^{+}$ions are shifted by $0.15 \mathrm{~cm}^{2} \mathrm{~s}^{-1}$ downwards, and the data for the $\mathrm{Cl}^{-}$ions are shifted by $0.15 \mathrm{~cm}^{2} \mathrm{~s}^{-1}$ upwards.

The top frame of the figure shows SDCs of ions for $\mathrm{CH}_{3}$ group terminated GNPs of different coverage. The data shows that the effect of nonpolar group terminated chains on both anions and cations is not very pronounced. The center frame shows SDCs of ions for $\mathrm{COO}^{-}$group terminated GNPs of different coverage. The data show that both the counter ions and coions are slowed down near the terminal group region. This effect is more pronounced for the counter ions than for the coions. The mobility of $\mathrm{Na}^{+}$ions is affected significantly in the region $\leq 3 \mathrm{~nm}$ from the center. The effect is smaller for $\mathrm{Cl}^{-}$ ions and it is pronounced only for the highest degrees of functionalization $\left(d_{f}=1\right)$. The bottom frame shows SDCs of ions for $\mathrm{NH}_{3}^{+}$group terminated GNPs of different coverage. The results are similar to the $\mathrm{COO}^{-}$group terminated systems only the role of $\mathrm{Na}^{+}$ and $\mathrm{Cl}^{-}$ions are reversed." [Ref. Journal of Physical Chemistry C, 2015, 119 (45), pp $25566-25575]$

Figure 4.12 (left) shows the average number of hydrogen bonds per polar terminal group. The right side of the figure 4.12 shows the average number of ion contacts per polar terminal groups. The analysis of the average number of hydrogen bonds and ionic contacts has been done calculating the total number of hydrogen bonds and ionic contacts over a $40 \mathrm{~ns}$ time scale and dividing it by the total number of terminal groups. The criteria of hydrogen bonds and ionic contacts are given in subsection 2.5.5.

The average number of hydrogen bonds and ion contacts per terminal groups show significant differences between two types of terminal groups $\left(\mathrm{COO}^{-}\right.$and $\left.\mathrm{NH}_{3}^{+}\right)$. Between the $\mathrm{COO}^{-}$terminal group and water, it varies between 1.5 and 2.5, and the number of hydrogen bonds between the $\mathrm{NH}_{3}^{+}$terminal groups and water varies between 0.5 and 1 . Thus the number of hydrogen bonds per terminal group of the $\mathrm{COO}^{-}$terminal group is more than 2 times larger than for the $\mathrm{NH}_{3}^{+}$terminal group. The number of contact ions per terminal group also differs significantly, but the values of ion contacts lie below 1 for both types of terminal groups. The number of hydrogen bonds per terminal group decreases with increasing grafting density but ion contacts increase with increasing grafting density, irrespective of the type of the terminal groups. At the high grafting density the terminal groups prefer to form ion contacts thereby reducing the number of hydrogen bonds. The number of contacts between $\mathrm{COO}^{-}$terminal and water is more than two times higher 

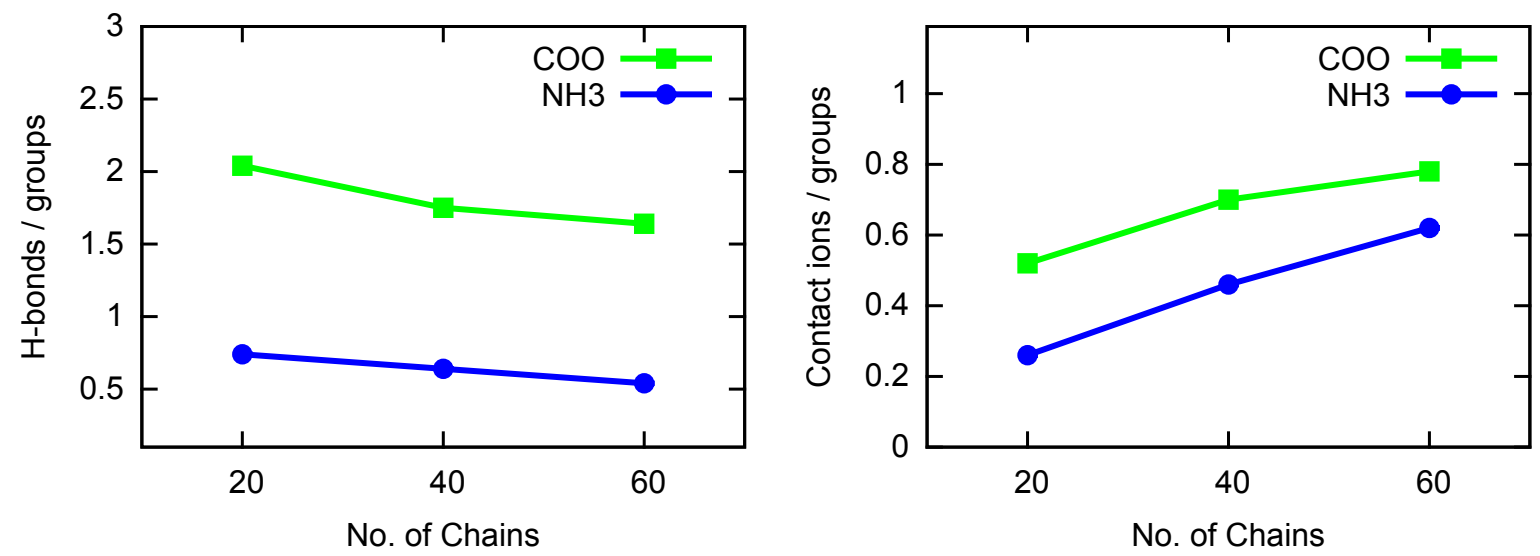

Figure 4.12: In the left hand side hydrogen bonds per polar terminal groups are shown as a function of the grafting density (here hydrogen bond data are divided by a factor 3). In the right hand side contact ions per polar terminal groups are shown as a function of the grafting density.

than the $\mathrm{NH}_{3}^{+}$and water, because each carboxyl oxygen is able to form several hydrogen bonds simultaneously. The number of counter ions in the simulation boxes for a certain grafting density are the same, so the number of ion contacts differs due to the water structure around the terminals and the mobility of the terminal groups. The self-diffusion coefficient of $\mathrm{COO}^{-}$and $\mathrm{NH}_{3}^{+}$terminal groups are $0.018\left(10^{-} 5 \mathrm{~cm}^{2} / \mathrm{s}\right)$ and $0.028\left(10^{-} 5\right.$ $\mathrm{cm}^{2} / \mathrm{s}$ ), respectively. So, the $\mathrm{Na}^{+}$ions stay in contact with the $\mathrm{COO}^{-}$terminals for longer time than the $\mathrm{Cl}^{-}$ions with $\mathrm{NH}_{3}^{+}$terminals.

\subsubsection{Integrated Charge and Water Orientation}

Figure 4.13 shows the radial charge integrated from the center of the GNP up to the distance $r$, separately for different components, for the COO-60 ( $\mathrm{COO}^{-}$terminated system with $\left.d_{f}=1\right)$ system (top-left) and the NH3-60 $\left(\mathrm{NH}_{3}^{+}\right.$terminated system with $\left.d_{f}=1\right)$ system (top-right), and the total integrated charge of the two systems (bottom). The individual contributions of cations and anions are similar for the two systems; however, the ionic charges (and thus the total amount of adsorbed ions) rise slightly more slowly with distance for the $\mathrm{NH}_{3}^{+}$terminated system. The contribution due to water orientation in the chain region cancels approximately, but water molecules near the polar terminal groups are oriented in such a way that they give net positive charge for COO-60 and 

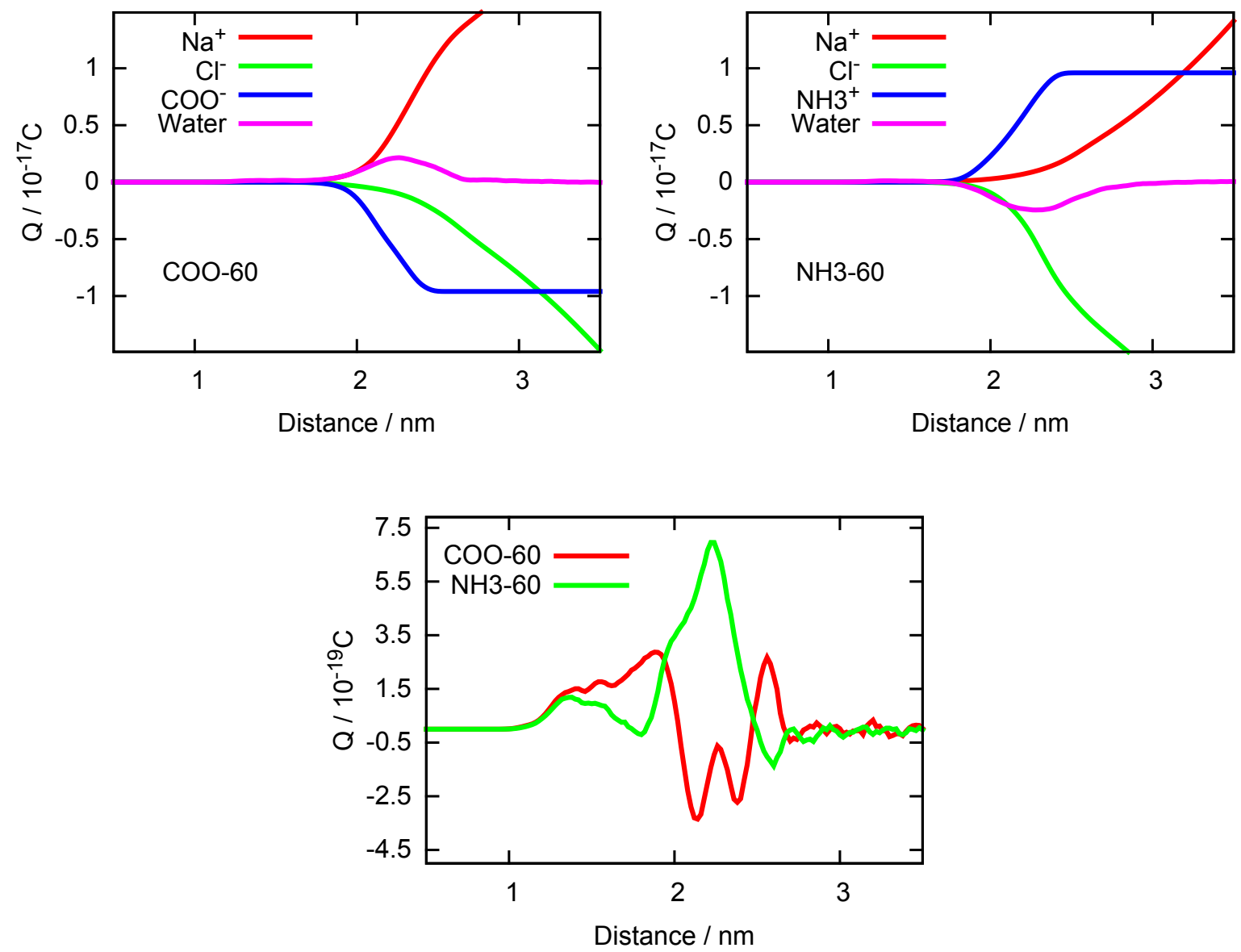

Figure 4.13: Radially integrated charge. Contribution of ions, terminal groups, and water of $\mathrm{COO}^{-}$group terminated GNP system with $d_{f}=1$ (top-left) and $\mathrm{NH}_{3}^{+}$group terminated GNP with $d_{f}=1$ (top-right). Bottom: Total integrated charge of both systems.

negative charge for NH3-60, and the peak width near the terminal is approximately $1 \mathrm{~nm}$. However, the total integrated water charge (bottom; note that the vertical scale is much smaller than for the individual contributions) close to the GNP is in both cases positive, indicating that water molecules in contact with the nanoparticle core are predominantly oriented with their hydrogen ends towards the core. Beyond approximately $3 \mathrm{~nm}$ from the gold core, the integrated charge is approximately zero. A detailed analysis of water orientation provides a clear view of water behavior and ordering in different regions of the systems.

The origin of the effective integrated charge near the solid liquid interface and near the polar terminal groups, and the effects of surfactants on water molecules can be ob- 


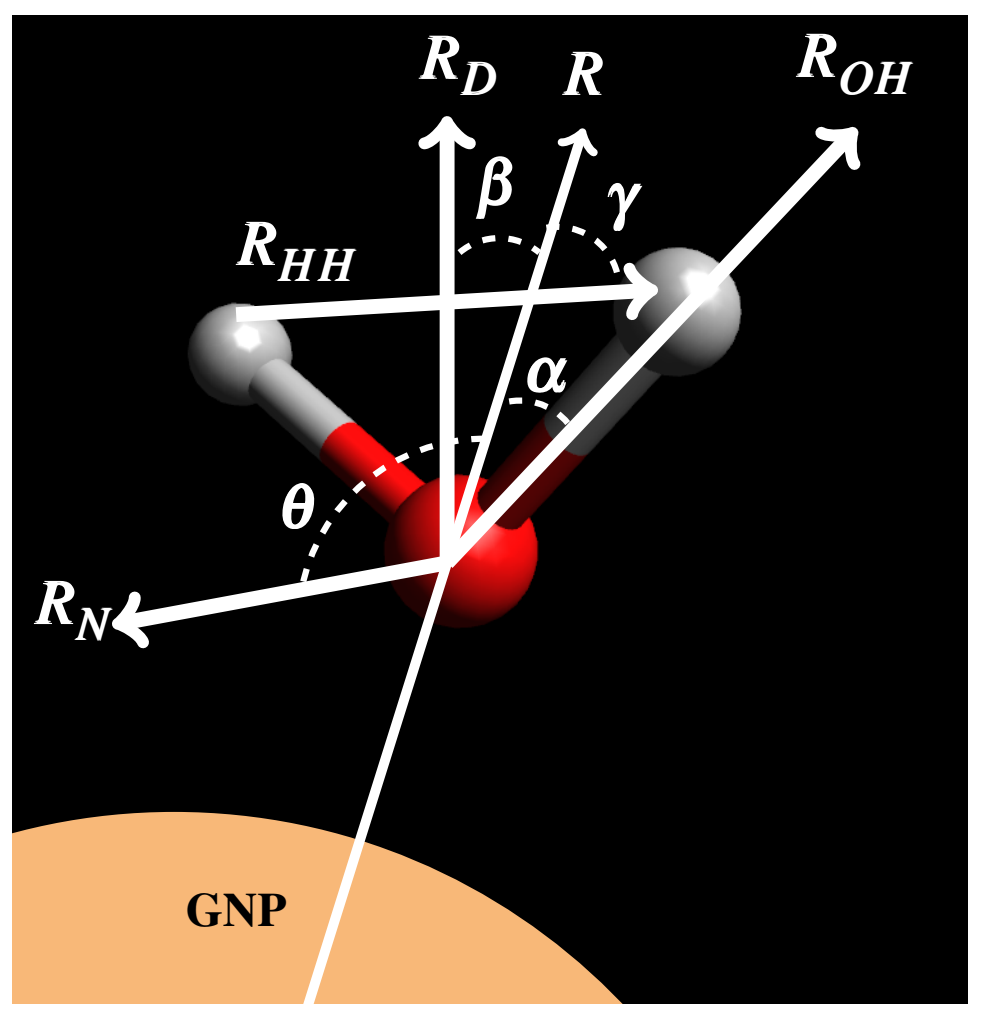

Figure 4.14: Dipole vector, $\mathrm{H}-\mathrm{H}$ vector, $\mathrm{O}-\mathrm{H}$ vector, normal vector, and the vector connecting the center of gold core and the oxygen of the water molecule are defined

tained by examining the orientation of the water molecules. To study the orientation of water molecules in three dimensions, four different vectors have been considered, namely the dipole vector $\left(\vec{R}_{D}\right)$, the vector connecting oxygen of a water molecule and a hydrogen atom of the same water molecule $\left(\vec{R}_{O H}\right)$, the normal vector $\left(\vec{R}_{N}\right)$ on the plane of water molecule and the vector connecting two hydrogen atoms of the water molecule $\left(\vec{R}_{H H}\right)$ (see Figure 4.14). The orientation angles are defined between these four vectors and the vector $(\vec{R})$ connecting the center of gold core and the oxygen atom of the water molecule. The angles between $\vec{R}$, and $\vec{R}_{D}, \vec{R}_{O H}, \vec{R}_{N}$ and $\vec{R}_{H H}$ are called $\beta, \alpha, \theta$ and $\gamma$, respectively (see Figure 4.14). The preference of orientation of water molecules from the surface of gold core towards the edge of box can be calculated by analyzing the probability of $\cos (\beta)$, $\cos (\alpha), \cos (\theta)$ and $\cos (\gamma)$. Water molecules have mirror like symmetry so, the vectors $\vec{R}_{N}$ and $\vec{R}_{H H}$ are indistinguishable in two opposite directions unlike the vectors $\vec{R}_{D}$ and $\vec{R}_{O H}$. Therefore, $\cos (\beta)$ and $\cos (\alpha)$ vary between -1 and +1 , and $\cos (\theta)$ and $\cos (\gamma)$ vary 
between 0 and +1 .

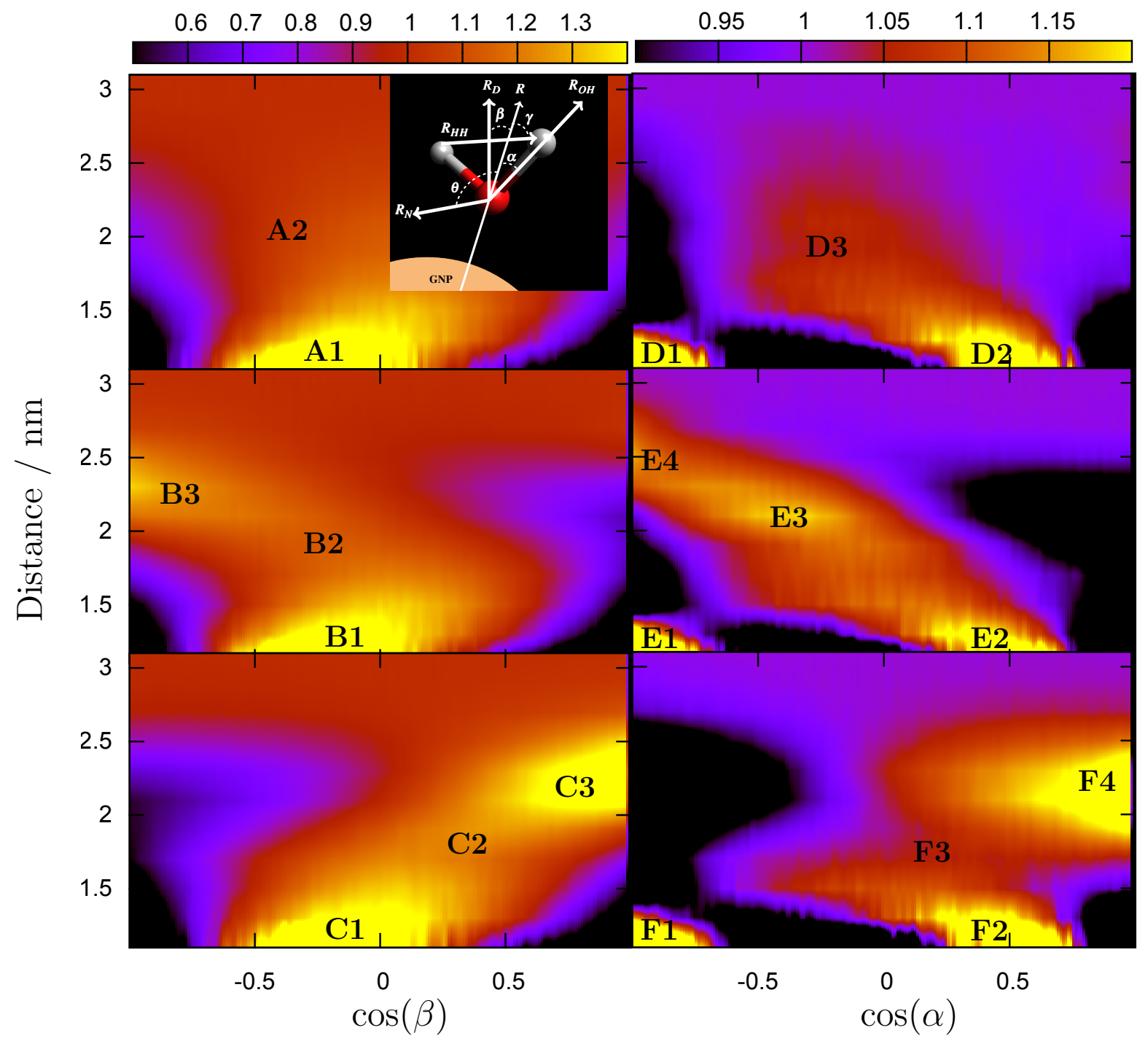

Figure 4.15: Left column: distribution of $\cos (\beta)$ as a function of distance from the center of the gold core towards the box edges of CH3-60 (top), COO-60 (center) and NH3-60 (bottom) systems. Right column: distribution of $\cos (\alpha)$ as a function of distance from the gold core of the CH3-60 (top), COO-60 (center) and NH3-60 (bottom) systems.

The water orientation analysis has been done only for the $d_{f}=1$ systems because different orientation probabilities are particularly prominent at high grafting density. Figure 4.15 shows the distribution of $\cos (\beta)$ (left column) and $\cos (\alpha)$ (right column) as a function of distance outward from the center of the gold core. From top to bottom, the 
figure shows the distribution for the $\mathrm{CH}_{3}$ (top), $\mathrm{COO}^{-}$(center) and $\mathrm{NH}_{3}^{+}$(bottom) terminated systems with $d_{f}=1$. In this figure the probability of the cosine angle orientations are represented by colors. The probability distribution of the cosine of the angles is calculated in concentric spherical shells of radii $\mathrm{r}$ and $\mathrm{r}+\delta \mathrm{r}$, where $\mathrm{r}$ varies from $1 \mathrm{~nm}$ to 3.2 $\mathrm{nm}$ and $\delta \mathrm{r}=0.2 \mathrm{~nm}$. For the $\mathrm{CH}_{3}$ group terminated system in figure 4.15 (top-left), the dipole vector has a preferential orientation near the gold water interface (see region A1) where $\cos (\beta) \approx-0.16$ or $\beta \approx 99^{\circ}$. This preference of orientation shifts towards $\beta \approx 90^{\circ}$ as the distance increases from the surface of the gold core and a uniform distribution is achieved after $\sim 2.4 \mathrm{~nm}$ (see figure 4.17 ). In case of the $\mathrm{COO}^{-}$group terminated system (center-left) at close distance from the surface of the gold core (see region B1), the dipole vector prefers to orient at $\cos (\beta) \approx-0.11$ or $\beta \approx 96^{\circ}$. As the distance increases from the surface, the preference of the orientation of the dipole vector also shifts towards $90^{\circ}$ like for the CH3-60 system. After $1.6 \mathrm{~nm}$ from the center (see region B2), the preference shifts towards $\cos (\beta)=-1$ or $\beta=180$. The dipole vector prefers to make an angle of $180^{\circ}$ near the negatively charged terminal groups at $2.3 \mathrm{~nm}$ from the center (see region B3) and the vector does not have any preference after $2.8 \mathrm{~nm}$ (see the figure 4.18). The preference of orientation near the gold surface of the $\mathrm{NH}_{3}^{+}$group terminated system (see region $\mathrm{C} 1)$ is $\cos (\beta) \approx-0.04$ or $\beta=92^{\circ}$ and the preference shifts towards $\cos (\beta)=1$ or $\beta=0^{\circ}$ with increasing distance from the surface (see region C2). At a distance of $2.3 \mathrm{~nm}$ from the center (see region C3) the $\vec{R}_{D}$ prefers to stay parallel to $\vec{R}$ (see figure 4.19 ).

The probability distribution of $\cos (\alpha)$ near the surface of gold core (see regions D1 and D2) of $\mathrm{CH}_{3}$ group terminated system (right top) shows two preferred orientations at $\cos (\alpha)=-1$ or $\alpha=180^{\circ}$ and $\cos (\alpha)=0.5$ or $\alpha=60^{\circ}$. As the distance increases from the surface of the gold core the preferred orientation of $\vec{R}_{O H}$ shifts from $60^{\circ}$ towards $80^{\circ}$ and this trend is observed up to $1.5 \mathrm{~nm}$ (see region D2). The probability distribution is not uniform over all $\alpha$ values in the hydrocarbon chain region (see region D3), rather it is a narrow channel. The $\vec{R}_{O H}$ vector does not have any preferred orientation after $3.0 \mathrm{~nm}$. The preference of orientation of $\vec{R}_{O H}$ for the COO-60 system (center right) near the gold surface (see regions E1 and E2) is similar to the preferred orientation of $\vec{R}_{O H}$ for the CH3-60 system (see regions D1 and D2). As the distance from the surface increases, the preferred orientation of $\vec{R}_{O H}$ shifts from $\cos (\alpha)=0.5$ near the surface of the gold core to $\cos (\alpha)=-1$ near the $\mathrm{COO}^{-}$terminal group at $2.5 \mathrm{~nm}$. This shift of preferred orientation is not abrupt, rather the orientation changes continuously with the distance from the surface (see regions E2, E3 and E4). Near the $\mathrm{COO}^{-}$terminal group $\vec{R}_{O H}$ tries to make 


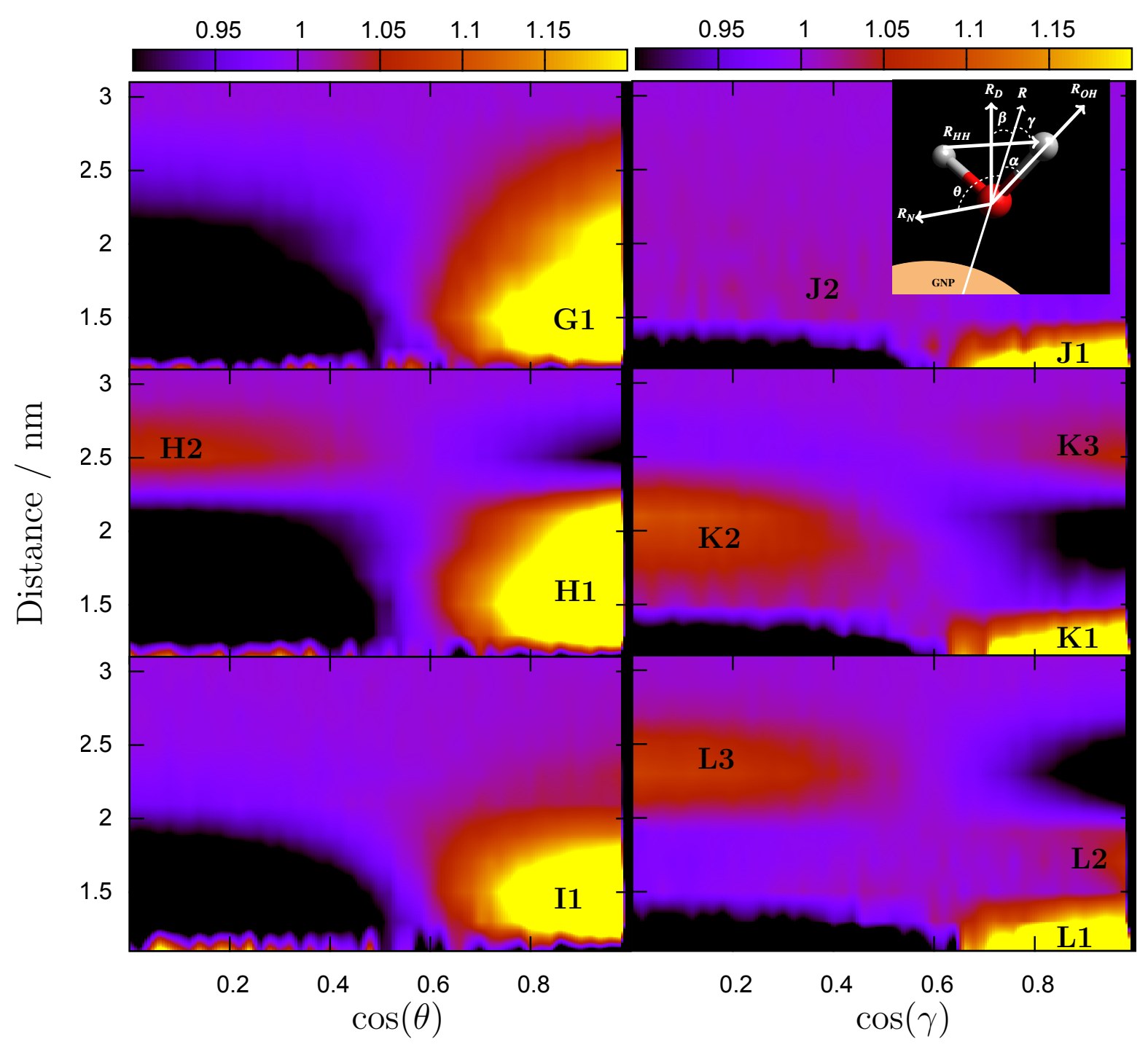

Figure 4.16: Left column: distribution of $\cos (\theta)$ as a function of distance from the center of the gold core towards the box edge of the CH3-60 (top), COO-60 (center) and NH3-60 (bottom) systems. Right column: distribution of $\cos (\gamma)$ of as a function of distance from the gold core of the CH3-60 (top), COO-60 (center) and NH3-60 (bottom) systems.

an angle of $180^{\circ}$ to $\vec{R}$ and after $3.0 \mathrm{~nm}$ from the center $\vec{R}_{O H}$ does not have any preferred orientation (see the figure 4.18). The preferred orientation of $\vec{R}_{O H}$ near the surface of the gold core (see regions F1 and F2) for the NH3-60 system (right bottom) is similar to the orientation of $\vec{R}_{O H}$ for the CH3-60 system. The preference of $\vec{R}_{O H}$ lies mostly between $\cos (\alpha)=-0.5$ and $\cos (\alpha)=1$ in the hydrocarbon chain region (see region F3). Near the $\mathrm{NH}_{3}^{+}$terminal group (see region F4) at $2.3 \mathrm{~nm}$ from the center, $\vec{R}_{O H}$ prefers to lie parallel 
to $\vec{R}$.

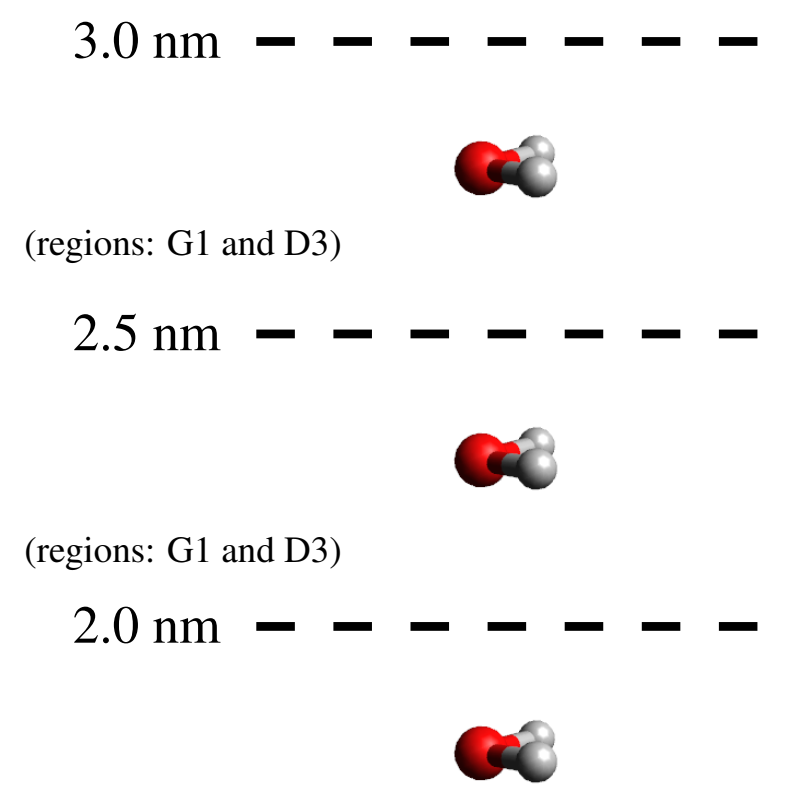

(regions: G1 and D3)

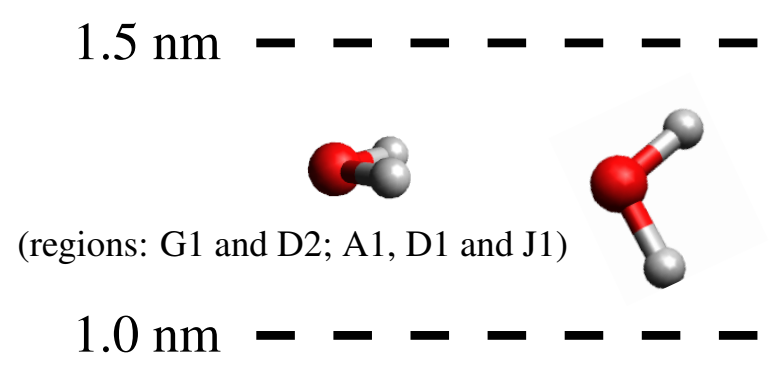

\section{CH3-60}

Figure 4.17: Sketch of water orientation in different shells from the surface of the gold core for CH3-60 system.

Figure 4.16 shows the distribution of $\cos (\theta)$ (left column) and $\cos (\gamma)$ (right column) as a function of the distance outward from the center of the gold core. From top to bottom, the figure shows the distributions for $\mathrm{CH}_{3}$ (top), $\mathrm{COO}^{-}$(center) and $\mathrm{NH}_{3}^{+}$(bottom) group terminated systems with $d_{f}=1$. In the CH3-60 system (top-left), $\vec{R}_{N}$ prefers to make an angle of $0^{\circ}$ with $\vec{R}$ in the region starting from the gold surface up to $2.8 \mathrm{~nm}$ (see region G1), and after $2.8 \mathrm{~nm} \vec{R}_{N}$ does not show any strong preference of orientation (see the figure 4.17). $\vec{R}_{N}$ for the COO-60 system (center-left) prefers to lie parallel with $\vec{R}$ in the 


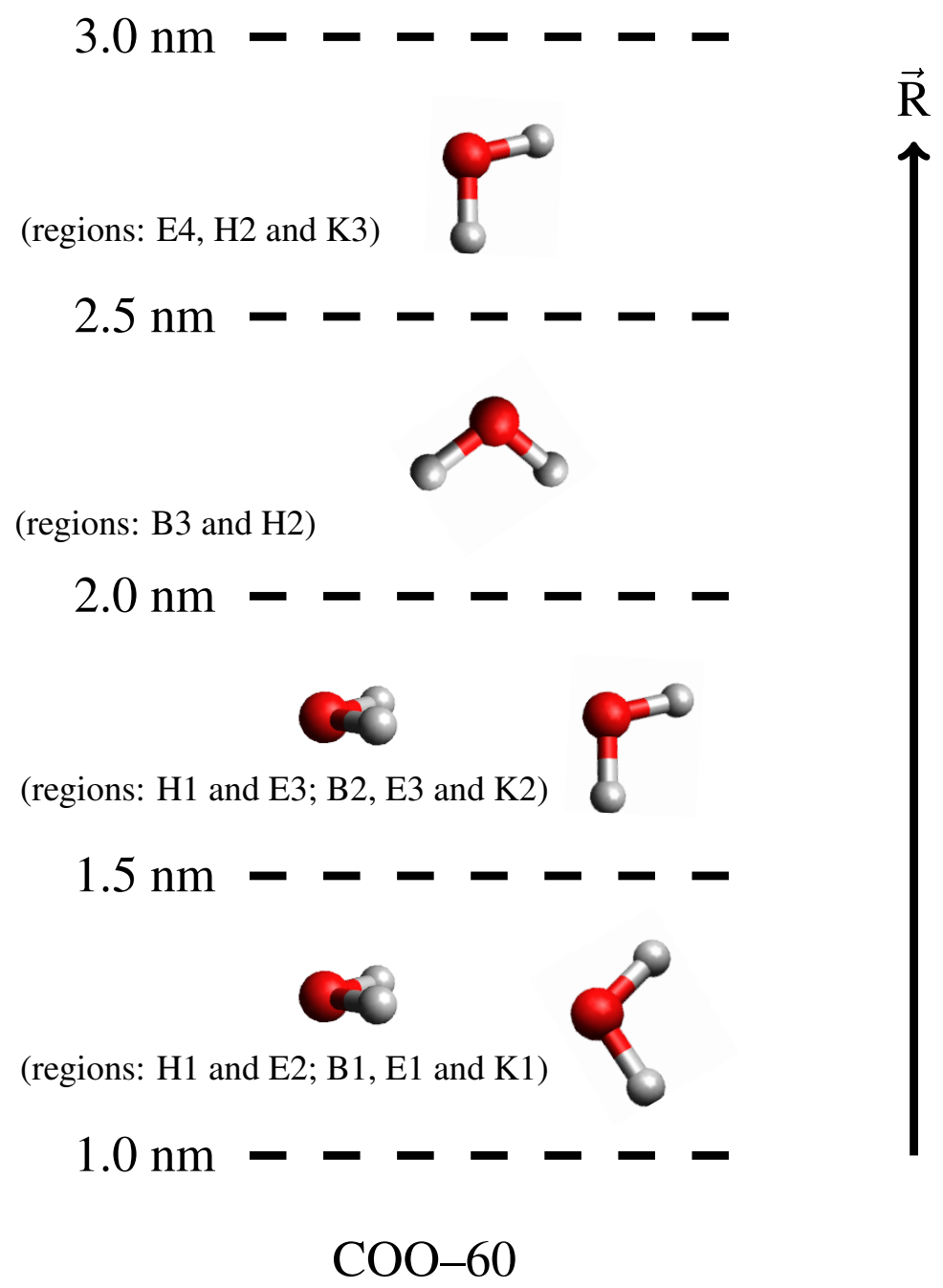

Figure 4.18: Sketch of water orientation in different shells from the surface of the gold core for COO-60 system.

region starting from the gold surface up to $2.3 \mathrm{~nm}$ (see region $\mathrm{H} 1$ ), and from $2.3 \mathrm{~nm}$ to $3.0 \mathrm{~nm}$ (see region H2) $\vec{R}_{N}$ prefers to make an angle between $60^{\circ}$ and $180^{\circ}$. After 3.0 $\mathrm{nm}, \vec{R}_{N}$ vector does not have any strong preference of orientation (see the figure 4.18). Similarly, for the NH3-60 system (bottom-left) $\vec{R}_{N}$ prefers to make an angle of $0^{\circ}$ with the $\vec{R}$ vector in the region starting from the surface up to $3.0 \mathrm{~nm}$ (see region I1).

In case of the CH3-60 system (top right), $\vec{R}_{H H}$ prefers to orient at $\cos (\gamma)=1$ or $\gamma=0^{\circ}$ in the region starting from the gold surface up to $1.5 \mathrm{~nm}$ (see region J1), which implies that $\vec{R}_{H H}$ lies preferentially in a direction parallel to the $\vec{R}$. $\vec{R}_{H H}$ does not have 
any strong preference after $1.5 \mathrm{~nm}$. The preferred orientation of $\vec{R}_{H H}$ for the COO-60 system (center right) in the region starting from the gold surface up to $1.5 \mathrm{~nm}$ (see region K1) is similar to the orientation of $\vec{R}_{H H}$ for the CH3-60 system. $\vec{R}_{H H}$ prefers to orient at $\cos (\gamma)=0$ or $\gamma=90^{\circ}$ in the region starting from $1.5 \mathrm{~nm}$ to $2.4 \mathrm{~nm}$ (see region $\mathrm{K} 2$ ), which implies $\vec{R}_{H H}$ prefers a direction perpendicular to $\vec{R}$ inside the hydrocarbon chains. Near the polar terminal group (see region K3), the $\vec{R}_{H H}$ preferentially lies parallel to $\vec{R}$. $\vec{R}_{H H}$ for the NH3-60 system (right bottom) in the region starting from the gold surface up to $2 \mathrm{~nm}$ (see regions L1 and L2) prefers to orient at $\cos (\gamma)=1$ or $\gamma=0^{\circ}$ which implies that $\vec{R}_{H H}$ lies preferentially in a direction parallel to $\vec{R}$. In the region starting from $2 \mathrm{~nm}$ to $2.8 \mathrm{~nm}$ (see region L3), $\vec{R}_{H H}$ prefers to orient at $\cos (\gamma)=0$ and $\vec{R}_{H H}$ does not have any preferred orientation after $3 \mathrm{~nm}$.

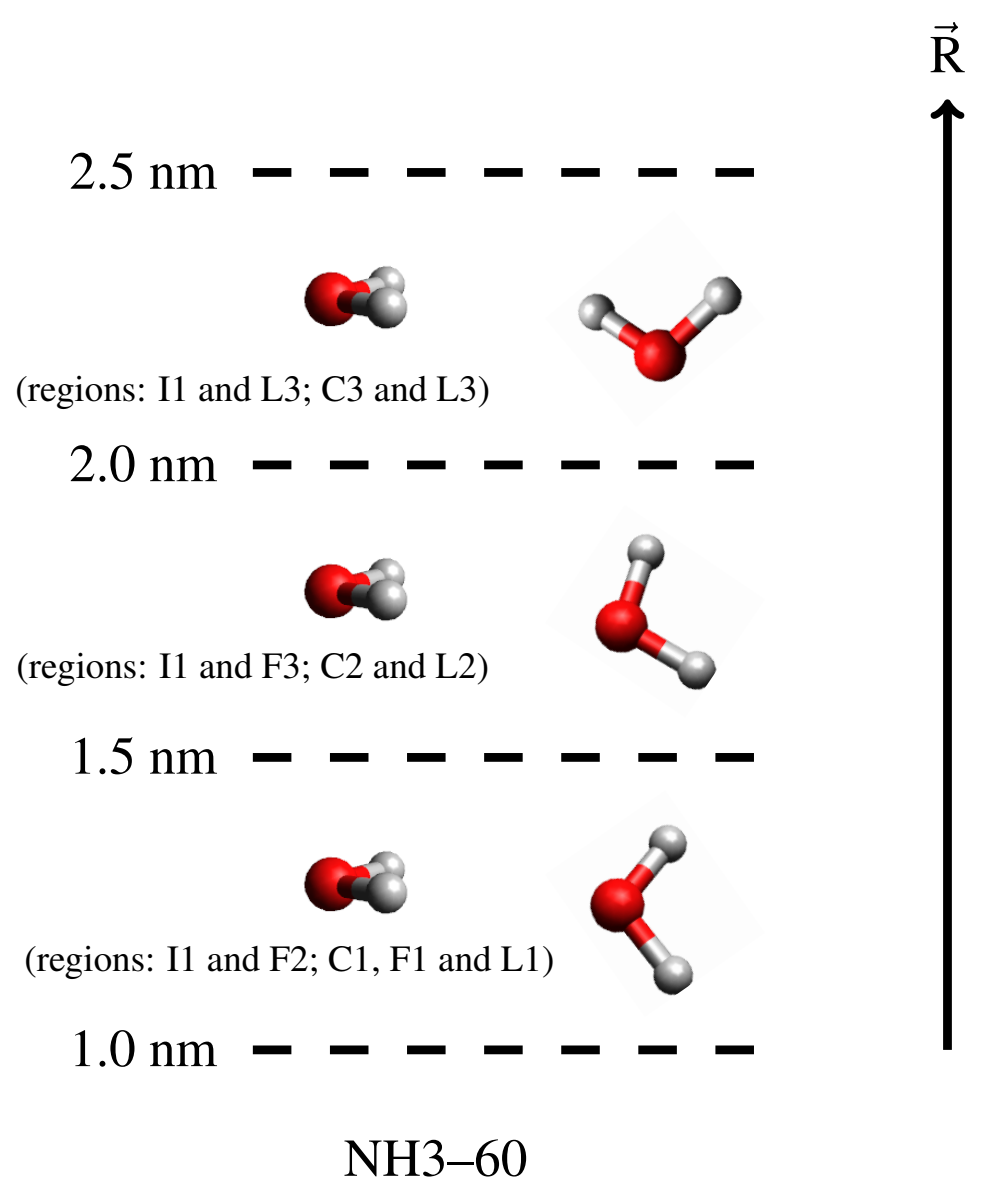

Figure 4.19: Sketch of water orientation in different shells from the surface of the gold core for NH3-60 system. 
The above results of water orientation show that there are two preferential orientation near the gold surface irrespective of the type of the systems. In one case, one hydrogen of a water molecule points towards the gold surface and in the other case the normal vector is parallel to the surface normal. This plane normal of water molecule always remains parallel to the surface normal up to $3.0 \mathrm{~nm}$ and after that water molecules orient randomly in all systems. Other type of orientation are observed, where the dipole vector $\left(\vec{R}_{D}\right)$ slowly rotates from $90^{\circ}$ to $180^{\circ}$ for COO-60 and from $90^{\circ}$ to $0^{\circ}$ for the NH360 systems in the hydrocarbon chain region. Considering all types of orientations in the different regions, the sketches of water orientation are given in figure 4.17 for CH3-60, figure 4.18 for COO-60 and figure 4.19 for NH3-60. The above preferential orientations of water molecules near the solid liquid interface, near the polar terminal group and inside the hydrocarbon chain region lead to an effective integrated charge at those places. 


\section{Coating Asymmetry on Gold Clus- ter and its Prevention}

In this chapter, simulations of linear and branched chain functionalized gold nanoparticles with varying chain length in aqueous $\mathrm{NaCl}$ solution are discussed. The focus is given on variation of coating asymmetry with linear chain length and prevention of coating asymmetry with branched chains. Attention is also given to surface access of the gold core and the solvent accessible surface area of the alkyl chains. Here, all force field parameters have been taken from the OPLS-AA force field for the simulations, unlike in the GNP simulations in the chapter 4, where a mixture of the AMBER-99 and the CHARMM-27 force field parameters has been used.

\subsection{System Preparation and Methods}

Functionalized GNPs (Figure 5.1) have three parts: the gold core (consisting of 114 gold atoms), the surface (S-Au-S) and the functionalized chains. The gold core and the surface are modeled as discussed in the chapter 4 . The shape and length of chains were changed to build different systems. In the first phase 12 different GNPs were modeled varying the linear chain length (between 5 and 23 carbon atoms) and the terminal groups $\left(\mathrm{NH}_{3}^{+}, \mathrm{CH}_{3}\right.$ and $\mathrm{COO}^{-}$). All the GNPs have full coverage (60 chains on the surface). In the second phase 10 different GNPs with half coverage (30 branched chains on the surface) have been prepared by varying the branched chain length (11 and 17 carbon atoms in a long chain), the junction position of chains, and the terminal groups $\left(\mathrm{NH}_{3}^{+}\right.$and $\left.\mathrm{CH}_{3}\right)$. Chains are branched at 4 th, 8 th and 12 th carbon atom from the surface of the gold core. The number of carbon atoms from the junction to both terminals is the same; see figure 5.2. The branched chain GNPs have a lower number of carbon atoms than the corresponding 


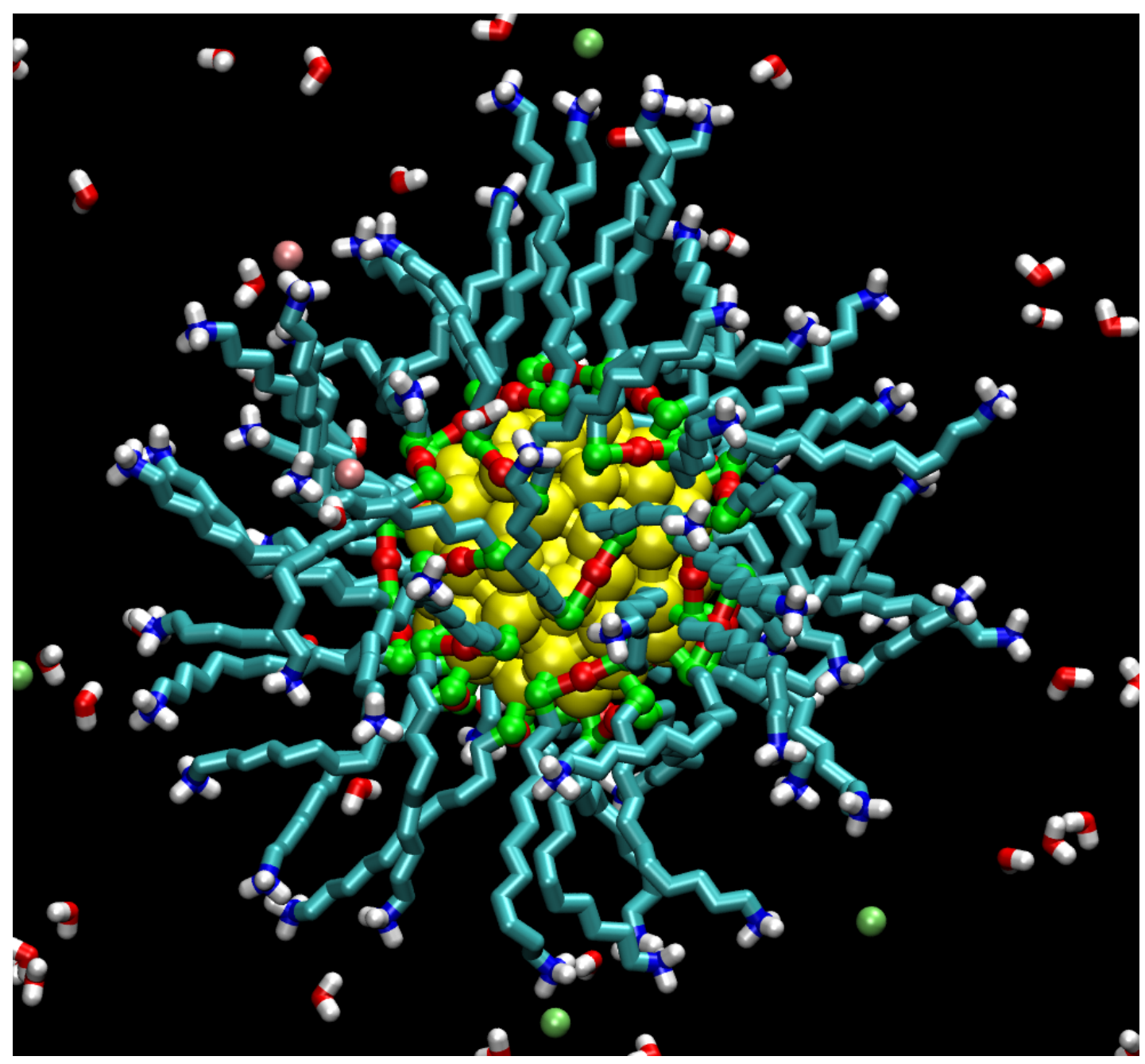

Figure 5.1: Fully functionalized GNP with $60-\left(\mathrm{CH}_{2}\right)_{11}-\mathrm{NH}_{3}^{+}$chains including some water (white and red), $\mathrm{Na}^{+}$(blue) and $\mathrm{Cl}^{-}$(pink) ions

GNPs with a linear chain, so as to keep the number of terminal groups equal to the linear chain GNPs. In the third phase, to keep the total number of carbon atoms the same as in the system having a junction at the 4 th carbon with $\mathrm{C}_{l}=17$, another 4 systems of $\mathrm{C}_{l}=17$ with junction at the 8 th and 12 th carbon atom have been prepared by attaching more branched chains on the surface of the gold cores. All systems are given in the diagram 5.1 at the end of this section. In diagram 5.1 the labels CL-5, CL-11, CL-17 and CL-23 indicate gold nanoparticles with chain length $\left(\mathrm{C}_{l}\right) 5,11,17$ and 23 , respectively. J-4, J-8 and J-12 represent the GNPs with branched chains and junctions at 4th, 8th and 12th carbon atom from the surface of the gold core. T-60, T-70 and $\mathrm{T}-80$ indicate that the 

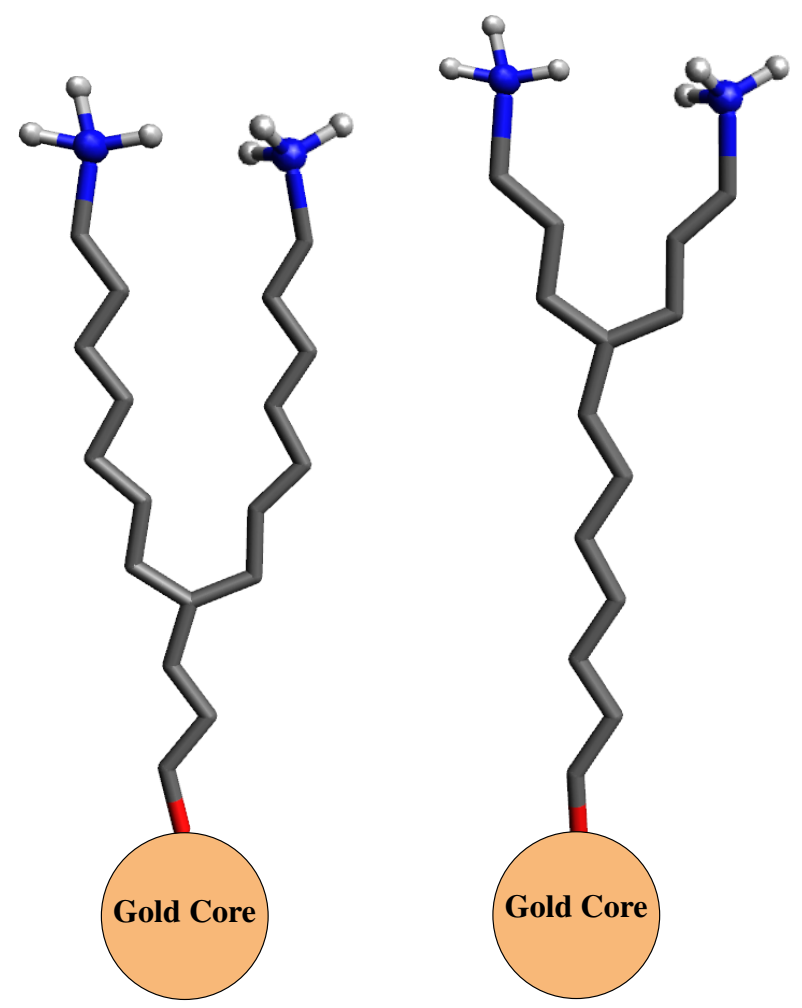

Figure 5.2: Schematic representation of $\mathrm{NH}_{3}^{+}$terminated branched chains with $\mathrm{C}_{l}=12$. Left: chain is branched at the 4th carbon atom from the surface of gold core. Right: chain is branched at the 8th carbon atom from the surface.

number of terminal groups are 60,70 and 80 , respectively.

Simulation box sizes are $6 \times 6 \times 6 \mathrm{~nm}^{3}, 8 \times 8 \times 8 \mathrm{~nm}^{3}, 10 \times 10 \times 10 \mathrm{~nm}^{3}$ and $12 \times 12 \times 12$ $\mathrm{nm}^{3}$ for the GNPs with $\mathrm{C}_{l}=5,11,17$ and 23 , respectively. The size of simulation boxes were varied depending on the chain length to save time. Simulation boxes contain approximately 6000, 15000, 30000 and 52000 water molecules for the GNPs with $\mathrm{C}_{l}=5$, 11, 17 and 23, respectively. Excess $\mathrm{Na}^{+}$and $\mathrm{Cl}^{-}$counter ions were added to make the systems charge neutral. In addition, 123, 295, 584 and $1017 \mathrm{NaCl}$ ion pairs were added as background electrolyte in the systems to obtain an approximate 1 molar solution. Description of all the systems are given in Table 5.1. In the table, $\mathrm{N}_{\text {terminal }}, \mathrm{N}_{w}, \mathrm{~N}_{+}$and $\mathrm{N}_{-}$indicate the number of terminal groups, number of water molecules, number of $\mathrm{Na}^{+}$ ions and number of $\mathrm{Cl}^{-}$ions, respectively.

The molecular editor Avogadro ${ }^{102}$ was used to construct the functionalized GNPs. 


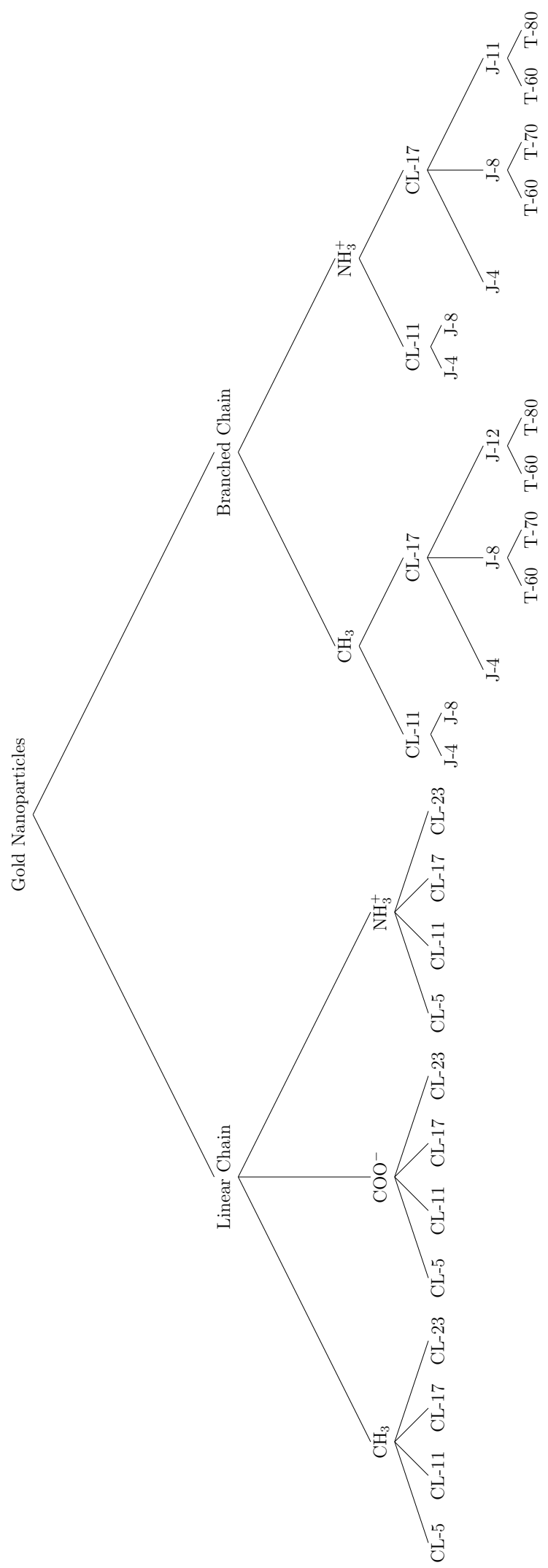

Diagram 5.1: All GNPs with linear and branched chains. 
Table 5.1: Summary of system description

\begin{tabular}{|c|c|c|c|c|c|c|c|}
\hline Terminal & $C_{l}$ & Box size $\left(\mathrm{nm}^{3}\right)$ & Branch atom & $\mathrm{N}_{\text {terminal }}$ & $\mathrm{N}_{w}$ & $\mathrm{~N}_{+}$ & $\mathrm{N}_{-}$ \\
\hline CH3 & 5 & $6 \times 6 \times 6$ & 0 th & 60 & 6176 & 123 & 123 \\
\hline CH3 & 11 & $8 \times 8 \times 8$ & 0 th & 60 & 15067 & 295 & 295 \\
\hline CH3 & 11 & $8 \times 8 \times 8$ & 4 th & 60 & 15163 & 297 & 297 \\
\hline CH3 & 11 & $8 \times 8 \times 8$ & 8 th & 60 & 15257 & 299 & 299 \\
\hline CH3 & 17 & $10 \times 10 \times 10$ & 0 th & 60 & 29991 & 584 & 584 \\
\hline CH3 & 17 & $10 \times 10 \times 10$ & 4 th & 60 & 30106 & 586 & 586 \\
\hline CH3 & 17 & $10 \times 10 \times 10$ & 8 th & 60 & 30184 & 588 & 588 \\
\hline CH3 & 17 & $10 \times 10 \times 10$ & 8 th & 70 & 30103 & 586 & 586 \\
\hline CH3 & 17 & $10 \times 10 \times 10$ & 12 th & 60 & 30280 & 589 & 589 \\
\hline CH3 & 17 & $10 \times 10 \times 10$ & 12 th & 80 & 30092 & 586 & 586 \\
\hline CH3 & 23 & $12 \times 12 \times 12$ & 0 th & 60 & 52618 & 1017 & 1017 \\
\hline NH3 & 5 & $6 \times 6 \times 6$ & 0 th & 60 & 6111 & 123 & 183 \\
\hline NH3 & 11 & $8 \times 8 \times 8$ & 0 th & 60 & 15004 & 295 & 355 \\
\hline NH3 & 11 & $8 \times 8 \times 8$ & 4 th & 60 & 15103 & 297 & 357 \\
\hline NH3 & 11 & $8 \times 8 \times 8$ & 8 th & 60 & 15202 & 299 & 359 \\
\hline NH3 & 17 & $10 \times 10 \times 10$ & 0 th & 60 & 29933 & 584 & 644 \\
\hline NH3 & 17 & $10 \times 10 \times 10$ & 4 th & 60 & 30035 & 586 & 646 \\
\hline NH3 & 17 & $10 \times 10 \times 10$ & 8 th & 60 & 30116 & 588 & 648 \\
\hline NH3 & 17 & $10 \times 10 \times 10$ & 8 th & 70 & 30040 & 586 & 646 \\
\hline NH3 & 17 & $10 \times 10 \times 10$ & 12 th & 60 & 30223 & 589 & 649 \\
\hline NH3 & 17 & $10 \times 10 \times 10$ & 12 th & 80 & 30038 & 586 & 646 \\
\hline NH3 & 23 & $12 \times 12 \times 12$ & 0 th & 60 & 52549 & 1017 & 1077 \\
\hline COO & 5 & $6 \times 6 \times 6$ & 0 th & 60 & 6116 & 183 & 123 \\
\hline COO & 11 & $8 \times 8 \times 8$ & 0 th & 60 & 15013 & 355 & 395 \\
\hline COO & 17 & $10 \times 10 \times 10$ & 0 th & 60 & 29936 & 644 & 584 \\
\hline COO & 23 & $12 \times 12 \times 12$ & 0 th & 60 & 52545 & 1077 & 1017 \\
\hline
\end{tabular}

$\mathrm{Na}^{+}$and $\mathrm{Cl}^{-}$ions were solvated in the water box with the help of VMD (1.9.1) ${ }^{88}$ employing the TopoTools plugin. Initially, functionalized GNPs were constructed and equilibrated in vacuum. Then, a sphere of water was cut out from a water box, and a GNP was placed inside that water box. After that, ions were added to the water box, and then the box was equilibrated for several ns.

The TIP3P ${ }^{87}$ water model has been used for all the simulations. The parameters for the functionalized chains have been taken from the OPLS-AA force field, and the parameters for the gold atoms were taken from Ref. ${ }^{104}$, since those are not available in 
the original OPLS-AA force field. All parameters are given in Table 5.2.

Table 5.2: Force field parameters

\begin{tabular}{|c|c|c|c|c|c|}
\hline \multicolumn{3}{|c|}{ Bonded interaction } & \multicolumn{3}{c|}{ Non-bonded interaction } \\
\hline Bond & $\mathrm{K}_{r}\left(\mathrm{Kcal} /\left(\mathrm{mol} / \AA^{2}\right)\right)$ & $\mathrm{r}_{0}(\AA)$ & Atom & $\epsilon($ Kcal$/ \mathrm{mol})$ & $\sigma(\AA)$ \\
\hline $\mathrm{C}-\mathrm{S}$ & 222.000 & 1.810 & $\mathrm{Au}$ & 1.322 & 2.951 \\
\hline $\mathrm{C}-\mathrm{C}$ & 268.000 & 1.529 & $\mathrm{~S}$ & 0.355 & 3.600 \\
\hline $\mathrm{C}-\mathrm{H}$ & 340.000 & 1.090 & $\mathrm{C}$ & 0.066 & 3.500 \\
\hline $\mathrm{C}-\mathrm{O}$ & 570.000 & 1.229 & $\mathrm{O}$ & 0.210 & 2.960 \\
\hline $\mathrm{C}-\mathrm{N}$ & 337.000 & 1.449 & $\mathrm{~N}$ & 0.170 & 3.250 \\
\hline
\end{tabular}

\begin{tabular}{|c|c|c|}
\hline \multicolumn{3}{|c|}{ Angle bend } \\
\hline Angle & $\mathrm{K}_{\theta}\left(\mathrm{Kcal} /\left(\mathrm{mol} / \mathrm{rad}^{2}\right)\right)$ & $\theta($ degree $)$ \\
\hline S-C-C & 50.00 & 147.7 \\
\hline C-C-C & 58.35 & 112.7 \\
\hline C-C-N & 80.00 & 109.7 \\
\hline C-C-C & 80.00 & 120.4 \\
\hline O-C-O & 80.00 & 126.0 \\
\hline C-N-H & 38.00 & 118.4 \\
\hline H-N-H & 35.00 & 120.0 \\
\hline
\end{tabular}

\begin{tabular}{|c|c|c|c|}
\hline \multicolumn{4}{|c|}{ Torsion } \\
\hline Dihedral & $\mathrm{K}_{1}(\mathrm{Kcal} / \mathrm{mol})$ & $\mathrm{K}_{2}(\mathrm{Kcal} / \mathrm{mol})$ & $\mathrm{K}_{3}(\mathrm{Kcal} / \mathrm{mol})$ \\
\hline S-C-C-C & 2.62 & -0.62 & 0.25 \\
\hline C-C-C-H & 0.00 & 0.00 & 0.30 \\
\hline H-C-C-H & 0.00 & 0.00 & 0.30 \\
\hline C-C-C-C & 1.30 & -0.05 & 0.20 \\
\hline C-C-C-N & 0.00 & 0.00 & 0.46 \\
\hline
\end{tabular}

All simulations are performed with the LAMMPS simulation package using the NVT ensemble, where the number of atoms, volume and temperature of the systems are held constant throughout the simulation. We ran the simulations at constant volume to avoid possible large fluctuations due to the big particle at the center of the box. Throughout the simulations, the gold core is fixed at the center of the simulation box. A NoséHoover thermostat ${ }^{59}$ has been used to keep the temperature of the systems constant at $300 \mathrm{~K}$ with a time constant of 1 ps. Several short runs of the order of picoseconds were performed using Berendsen and Nosé-Hoover thermostats (repeatedly) to reach the equilibrium more quickly. After several short runs, a long run of $2 \mathrm{~ns}$ has been performed at constant pressure (NPT ensemble) of 1 atmosphere to adjust the volume of the box. Production runs were performed for $80 \mathrm{~ns}$ with time step of 2 fs. A skin distance for 
the neighbor tables of $0.2 \mathrm{~nm}$ is updated in every 5 steps. The cut off of the LennardJones interaction has been taken as $1 \mathrm{~nm}$. In addition, the particle-particle-particle-mesh (PPPM) approach was introduced to calculate long range Coulomb interactions with a relative accuracy of $10^{-3}$. The SHAKE algorithm ${ }^{90}$ has been used to constrain $\mathrm{O}-\mathrm{H}$ bonds and $\mathrm{H}-\mathrm{O}-\mathrm{H}$ angles.

\subsection{Results and Discussion}

\subsubsection{Chain Length Dependence of Coating Asymmetry}

Figure 5.3 shows the snapshots of all linear chain functionalized systems. Ions, water and hydrogens of hydrocarbon chains are not shown in the snapshots to get a clearer view of the chain arrangement. All the snapshots were taken at the end of $80 \mathrm{~ns}$ production runs. In the picture, chain lengths $\left(\mathrm{C}_{l}\right)$ of the systems increase from top to bottom: $\mathrm{C}_{l}$ $=5,11,17$ and 23. From left to right, the terminal group changes: $\mathrm{CH}_{3}, \mathrm{COO}^{-}$and $\mathrm{NH}_{3}^{+}$. The GNPs with $\mathrm{C}_{l}=5$ do not show any coating asymmetry. Chains on the gold surface are isotropically distributed and the terminal groups are stretched outward from the gold cores. This symmetric distribution of short alkaline chains $\left(\mathrm{C}_{l}=5\right)$ on the gold cores does not depend on the nature of the terminal group. In the second row of figure 5.3, systems show mixed behavior depending on the terminal groups. The $\mathrm{CH}_{3}$ group terminated system with $\mathrm{C}_{l}=11$ shows bunching of alkyl chains, which leads to coating asymmetry on the gold surface, but the $\mathrm{COO}^{-}$and $\mathrm{NH}_{3}^{+}$groups terminated systems with $\mathrm{C}_{l}=11$ do not show this asymmetry. The polar group $\left(\mathrm{COO}^{-}\right.$and $\left.\mathrm{NH}_{3}^{+}\right)$terminated chains can not form bunches due to the strong electrostatic repulsion between the terminal groups, whereas the nonpolar group terminated chains do not interact electrostatically to prevent bunching. The systems with long chains $\left(\mathrm{C}_{l}=17\right.$ and 23$)$ show coating asymmetry irrespective of their terminal groups.

The coating asymmetry for the GNPs with long chains can be analyzed very easily using the time averaged angle distribution. The distribution provides the information about the bunching point where the chains start to form bundles and the nature of bunching. In the time averaged angle distribution, the angles have been calculated between all pairs of vectors which connect one sulfur atom and a carbon atom of a chain connected to the sulfur (see figure 5.4), and then the distributions of angles are averaged and normal- 


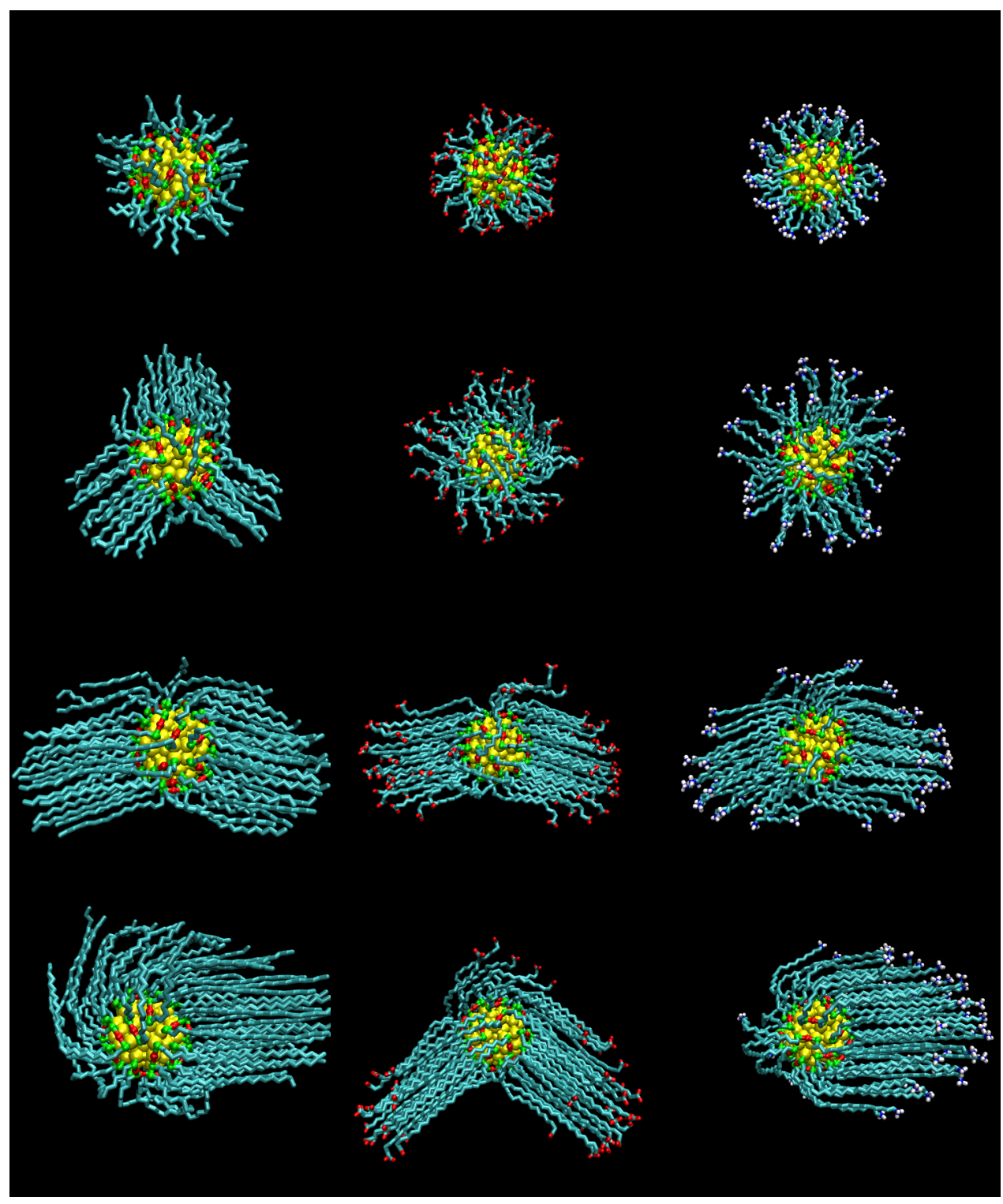

Figure 5.3: Snapshots of linear chain functionalized gold nanoparticles. From top to bottom, chain length increases: $\mathrm{C}_{l}=5,11,17$ and 23. From left to right, the terminal group changes in the order $\mathrm{CH}_{3}, \mathrm{COO}^{-}$and $\mathrm{NH}_{3}^{+}$.

ized over time. Figure 5.5 shows the time averaged angle distribution (TAAD) of different GNPs. The terminal groups of the GNPs change from top to bottom in the figure as $\mathrm{CH}_{3}$, $\mathrm{COO}^{-}$and $\mathrm{NH}_{3}^{+}$. The left and right columns of the figure are for the GNPs with $\mathrm{C}_{l}=11$ and $\mathrm{C}_{l}=23$, respectively. Along the vertical direction the carbon number changes. The carbon which is connected to the sulfur atom directly via a bond, considered as number 


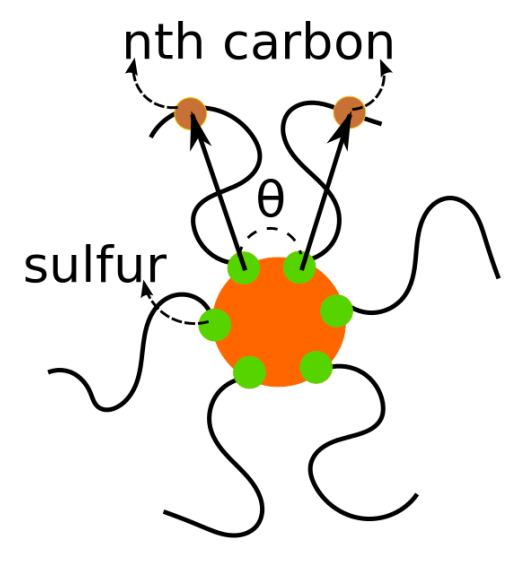

Figure 5.4: Schematic representation of angle for time average angle distribution. Vectors are defined between sulfur atoms and the nth carbon atoms.

one. The time averaged angle distribution (TAAD) of $\mathrm{CH}_{3}$ group terminated system with $\mathrm{C}_{l}=11$ shows two distinguishable separate bright yellow spots at high carbon numbers and this indicates coating asymmetry at high carbon number. The bright spots are distinguishable after the 7 th carbon atom from the gold surface, so the alkyl chains start to form bundles after the 7 th carbon atom from the surface. The TAAD of $\mathrm{COO}^{-}$and $\mathrm{NH}_{3}^{+}$ group terminated GNPs with $\mathrm{C}_{l}=11$ systems (left-center and left-bottom) do not show any separate bright spots, and this indicates that the chains are isotropically distributed over the gold cores.

The distributions for $\mathrm{CH}_{3}$ and $\mathrm{COO}^{-}$terminated GNPs with $\mathrm{C}_{l}=23$ (top-right and center-right) show discontinuous distribution of color and display two distinct high probability regions at high carbon atom numbers. The discontinuity starts after the 7th carbon atom from the gold surface for both systems. So, the systems have asymmetric coating and the bunching of the chains starts after the 7th carbon atom. The TAAD for $\mathrm{NH}_{3}^{+}$group terminated system with $\mathrm{C}_{l}=23$ (right-bottom) shows a high probability around $80^{\circ}$ at low carbon number and after around the 7th carbon number the high probability region moved to $\approx 20^{\circ}$. So, the chains start to form a single bunch after 7 th carbon atom in $\mathrm{NH}_{3}^{+}$group terminated system with $\mathrm{C}_{l}=23$. The TAAD for the GNPs with $\mathrm{C}_{l}=5$ and 17 are given in appendix A.3.

Coating asymmetry does not appear for the GNPs with short chains, but the GNPs with long chains show coating asymmetry. The coating asymmetry arises mainly due to hydrophobicity and free volume available to the chains. In case of short chains, the 

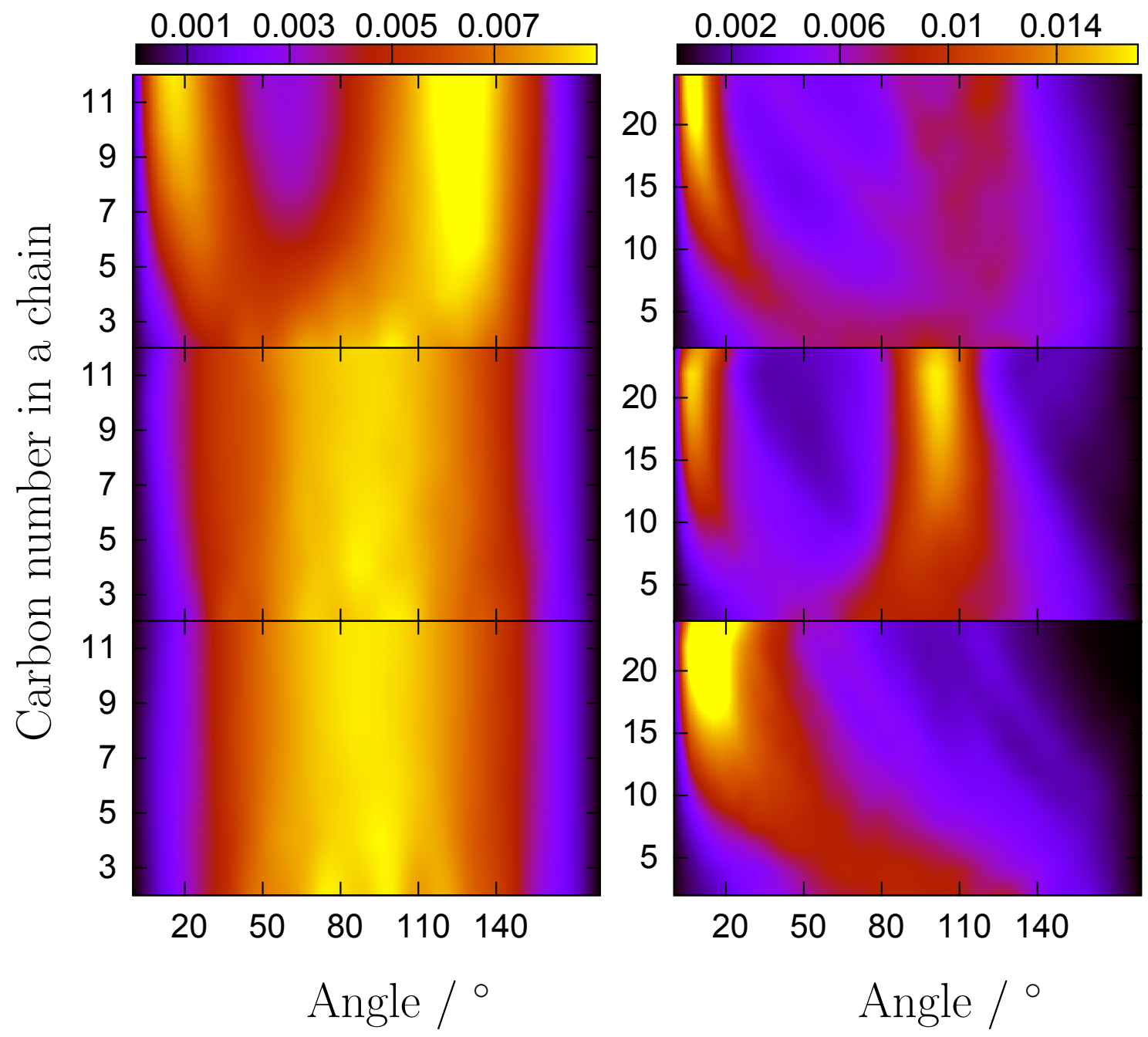

Figure 5.5: Time averaged angle distribution of $\mathrm{CH}_{3}, \mathrm{COO}^{-}$and $\mathrm{NH}_{3}^{+}$groups terminated systems (top to bottom). Left and right columns are for the GNPs system with $\mathrm{C}_{l}=11$ and $\mathrm{C}_{l}=23$, respectively.

chain volume is close to the free volume available to the chain, so the chains are uniformly distributed over the the gold core and this uniform distribution helps to keep the hydrophobic region of one chain in touch with another chain. For the long chains, the free volume close to the surface of the gold core (up to 7th carbon atom) is nearly equal to the volume of the chains but at long distance from the surface (after the 7th carbon atom), the free volume is much larger than the volume occupied by the chains. At long 
distances, the uniform distribution of chains would expose the hydrophobic regions to water. To avoid this phenomenon, the chains form bundles (or a bundle) to minimize the hydrophobic region which is exposed to water. The bundles of long chains are not of equal size, and the size is limited. The limitation of bundle size is entirely due to the gold core modeling and the constraint which connects the chains with the gold core. This bundle formation of long chains is not a computational artifact. The phenomenon has also been observed on the basis of the light scattering experiment. ${ }^{103}$

\subsubsection{Branched Chains and Prevention of Coating Asymmetry}

The coating asymmetry of long linear chains can be prevented by introducing branched alkyl chains. Figure 5.6 shows snapshots at the end of 80 ns production runs of branched chain coated gold nanoparticles with $\mathrm{C}_{l}=11$. The left hand side of the figure shows $\mathrm{CH}_{3}$ group terminated GNPs and the right hand side shows $\mathrm{NH}_{3}^{+}$terminated GNPs. From the top to the bottom of the figure, the junction points of branched chains change as 0th, 4th and 8th carbon. A branched chain with branch at 0th carbon is a linear chain. The $\mathrm{CH}_{3}$ group terminated GNP with junction at the 0th carbon and $\mathrm{C}_{l}=11$ can be abbreviated as CH3-J0-CL11-T60 for the benefit of discussion. The first, second, third and fourth terms of the abbreviation indicate terminal group, junction point, chain length and number of terminal group of the GNP. Other systems will also be defined in the same manner. In figure 5.6, the CH3-J0-CL11-T60 system (top left) shows asymmetric coating but CH3-J4-CL11-T60 (center left) and CH3-J8-CL11-T60 (bottom left) do not show coating asymmetry. On the right hand side of the figure, the NH3-J0-CL11-T60 (top right), NH3-J4-CL11-T60 (center right) and NH3-J8-CL11-T60 (bottom right) systems do not show any coating asymmetry. In case of the $\mathrm{CH}_{3}$ group terminated GNPs, as the junction point shifts from zero to higher values, alkyl chains break bundles and fall on the gold core to wrap it tightly. In case of NH3-J0-CL11-T60 system, the alkyl chains are not long enough to overcome the effect of electrostatic interaction between the polar terminal groups to form bundles. As the alkyl chains of NH3-J0-CL11-T60 system are symmetrically distributed, so the effect of shifting of junction points on the coating structure is not visible. This phenomenon can be seen clearly for both the polar and nonpolar groups terminated chains which are longer than $\mathrm{C}_{l}=11$.

Figure 5.7 shows snapshots at the end of 80 ns production runs of branched chain coated gold nanoparticles with $\mathrm{C}_{l}=17$. Left side and right side of the figure shows $\mathrm{CH}_{3}$ 


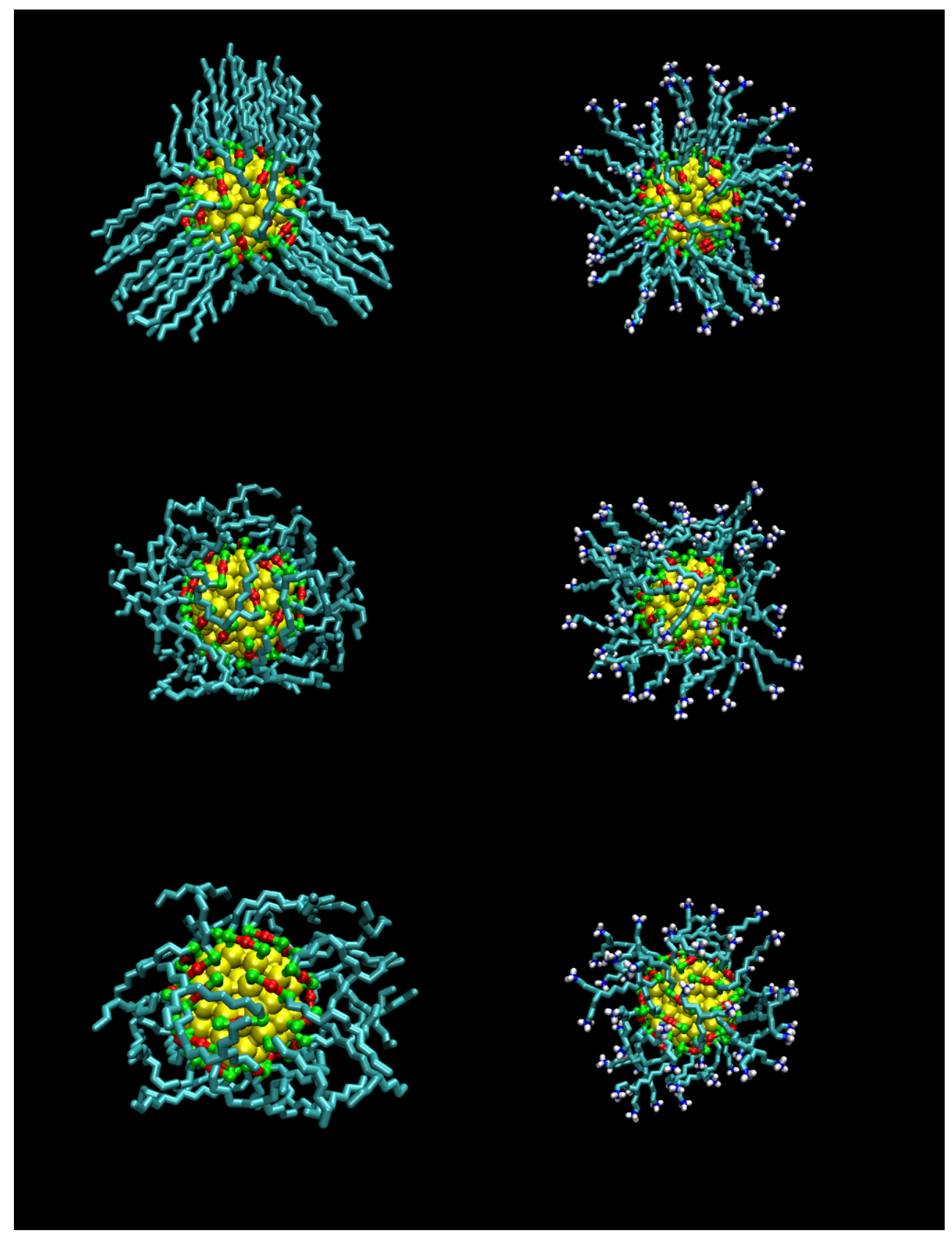

Figure 5.6: Snapshots of branched chain coated gold nanoparticles with $\mathrm{C}_{l}=11$. From the left to the right, terminal group changes: $\mathrm{CH}_{3}$ and $\mathrm{NH}_{3}^{+}$. Top to bottom, junction point changes: 0th, 4th and 8th carbon.

and $\mathrm{NH}_{3}^{+}$group terminated GNPs. From top to bottom of the figure, the junction points of the branched chains change as 0th, 4th, 8th and 12th carbon atom. CH3-J0-CL17-T60 (top left) and NH3-J0-CL17-T60 (top-right) systems show coating asymmetry. As the 

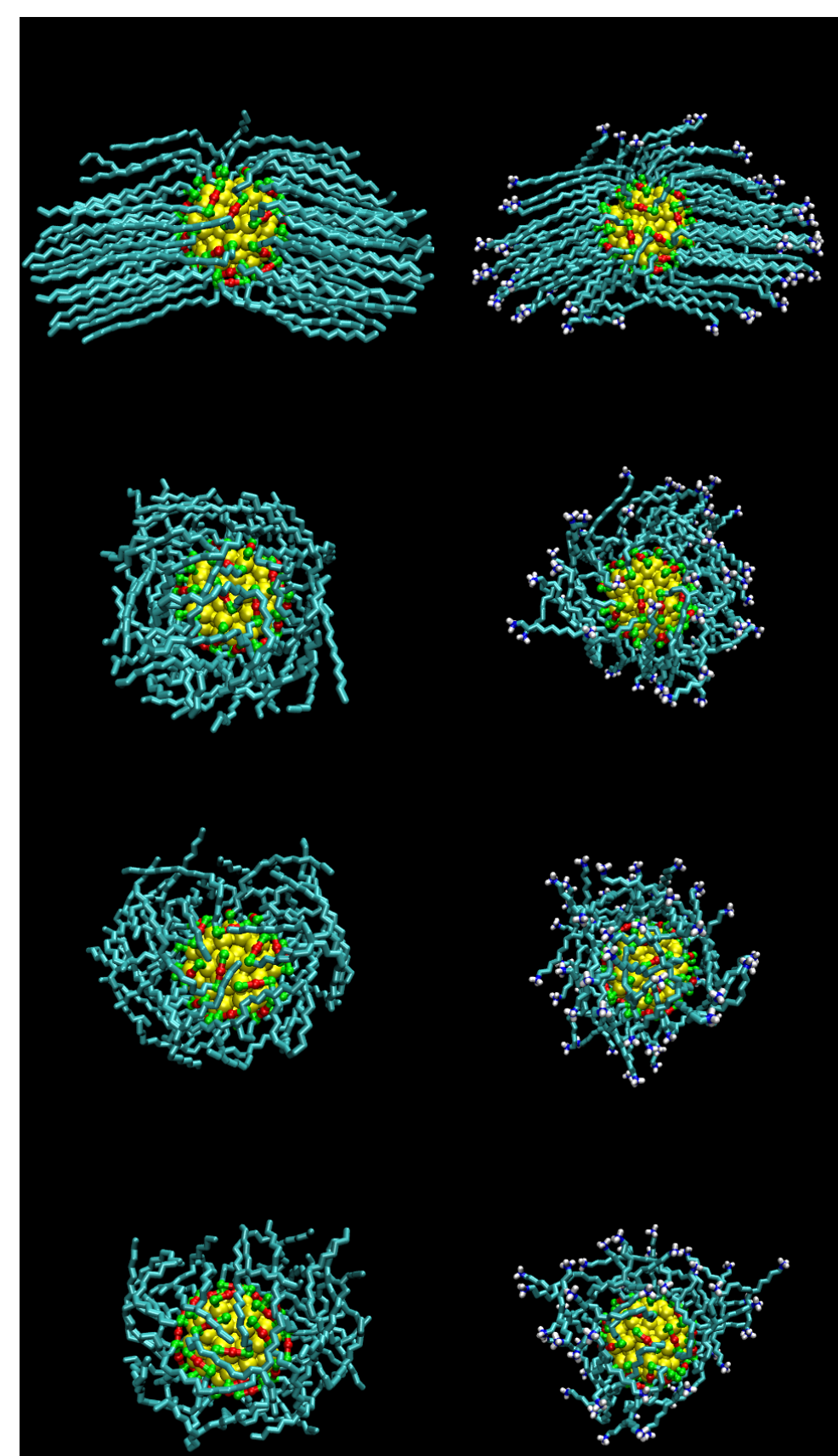

Figure 5.7: Snapshots of branched chain coated gold nanoparticles with $\mathrm{C}_{l}=17$. From the left to the right, terminal group changes: $\mathrm{CH}_{3}$ and $\mathrm{NH}_{3}^{+}$. Top to bottom, junction point changes: 0th, 4th, 8th and 12 th carbon. 
junction point moves from zero to higher values, the coating asymmetry becomes invisible for the systems irrespective of their terminal groups. Alkyl chains with junction points at the 4th, 8th and 12th carbon atom fall back on the gold core and try to wrap the core tightly.

Figure 5.8 shows the time averaged angle distribution (TAAD) of different branched chain coated GNP systems with $\mathrm{C}_{l}=17$. The left and right hand sides of the figure show the TAAD of $\mathrm{CH}_{3}$ and $\mathrm{NH}_{3}^{+}$group terminated GNPs, respectively. From top to bottom of the figure, the junction points of branched chains change as 0th, 4th, 8th and 12th carbon atom. The TAAD of the CH3-J0-CL17-T60 system (top left) show two separate bright yellow spots after the 6th carbon atom. This indicates that chains start to form bundles after the 6th carbon atom from the surface of the gold core. Near the surface (up to the 6th carbon atom) chains are symmetrically distributed due to physical constraints. The TAAD of CH3-J4-CL17-T60 (second row left), CH3-J8-CL17-T60 (third row left) and CH3-J12-CL17-T60 (bottom left) do not have any separate bright spots, so the systems do not have any type of coating asymmetry during the whole $80 \mathrm{~ns}$ simulation time. The TAAD of NH3-J0-CL17-T60 (top-right) has two distinguishable bright spots at high carbon number. The color distribution of the NH3-J0-CL17-T60 system has separate spots after the 6th carbon atom and this indicates that the system forms bundles after the 6th carbon atom. The TAADs of other systems with $\mathrm{NH}_{3}^{+}$terminal groups show continuous broad color distributions all over the length of the branched chains. These results for the branched chain systems $\left(C_{l}=17\right)$ point out that as the junction shifts from zero to the higher values, the coating asymmetry vanishes.

Linear long chain coated GNPs easily forms bundles, which leads to coating asymmetry. The polar terminal groups can prevent the asymmetry up to a certain chain length $\left(\mathrm{C}_{l}=11\right)$. The prevention of asymmetry is important to prevent the exposition of the core to the solution, particularly if the core consists of an active metal. Apart from this, the solubility of GNPs can be controlled by tuning the shape of the chains. The coating asymmetry can be prevented and the shape of chains can be tuned by replacing the linear chains with the branched chains (except the branched chains junction at 0th carbon). Branched chains prevent coating asymmetry irrespective of the junction positions. Branched chains have physical constraints which force to make a certain angle at the junction point between two chains and this prevents the chains to come close together to form bundles. The hypothesis can be made from here that the coating asymmetry can be seen again for branched chain GNP if the chains are very long ( $>35$ carbon) and 

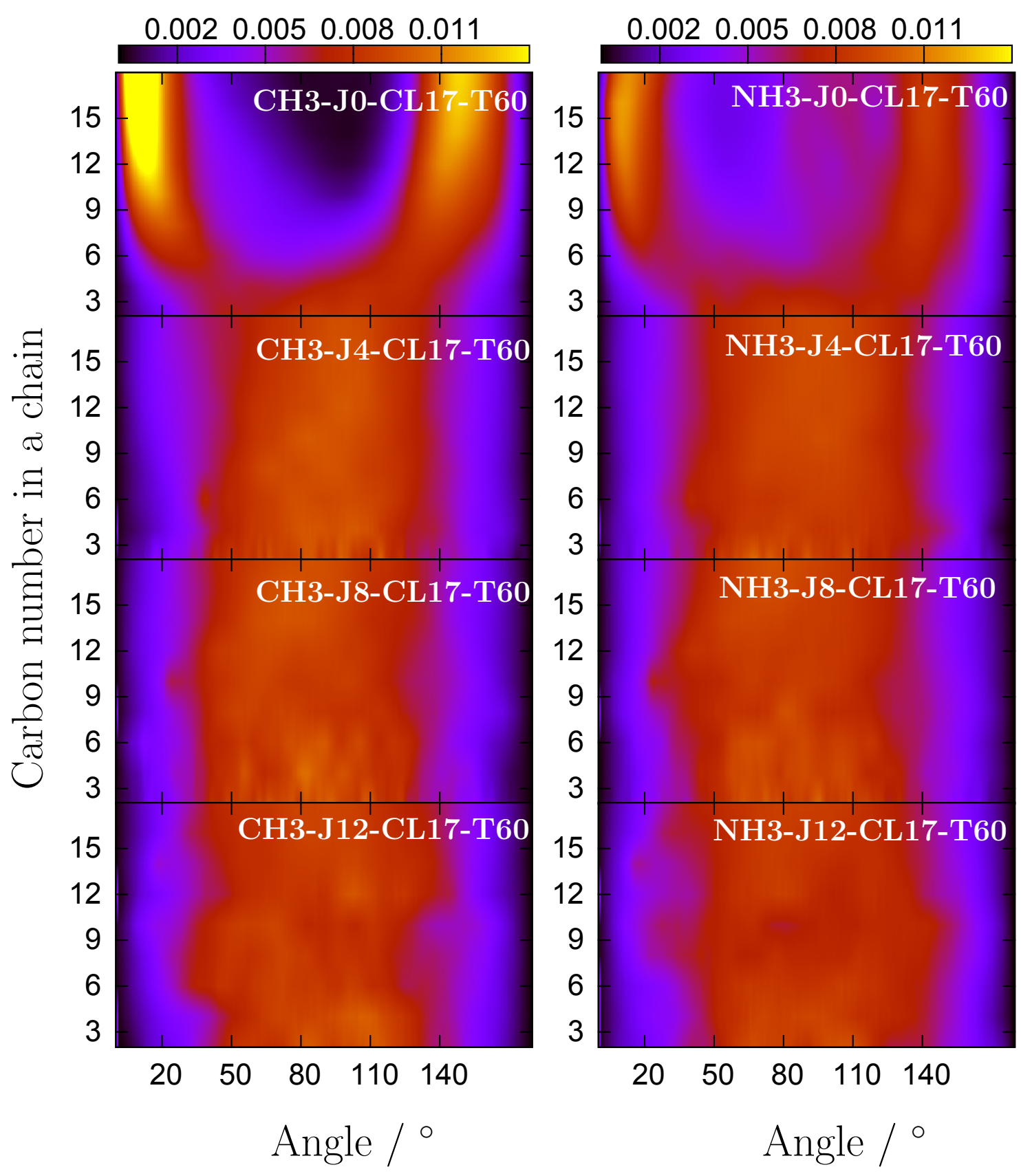

Figure 5.8: Time averaged angle distribution of $\mathrm{CH}_{3}$ and $\mathrm{NH}_{3}^{+}$group terminated systems (left to right). From top to bottom: the branched chain coated GNP systems with junction at 0 th, 4th, 8th and 12 th carbon. 
branching point is at a low carbon number (such as 4th carbon).

\subsubsection{Surface Access of GNPs}

Figure 5.9 shows color coded maps of the distribution of closest atoms (oxygen of water and carbon of hydrocarbon chains) to the GNP surface for the linear chain-coated GNPs. Along the horizontal direction the azimuthal angle $\phi$ varies between $0^{\circ}$ and $360^{\circ}$. Along the vertical direction the cosine of the polar angle $\theta$ varies between -1 and +1 . The largest value 1 (yellow) in these maps indicates that the oxygen of a water molecule is always closest to the gold surface, and a value 0 (purple) indicates that a carbon atom of chains is always closest to the surface. The left column shows maps for $\mathrm{CH}_{3}$ group-terminated linear chains with chain length of $5,11,17$ and 23 carbon atoms (from top to bottom). The right column shows analogous data for $\mathrm{NH}_{3}^{+}$group-terminated linear chains with 5, 11, 17 and 23 carbon atoms. The variation of colors is to some extent a consequence of the limited sampling time of $80 \mathrm{~ns}$, but also a consequence of the not entirely isotropic distribution of anchoring points for the chains.

The short chains (top row) point predominantly outward from the GNPs. Water access to the gold surface is somewhat reduced for the nonpolar chains, which tend to fold and cover the nanoparticle surface. For the polar chains, which are more likely to be extended and pointing away from the gold core the water access is higher. The folding of nonpolar chains of $\mathrm{C}_{l}=11$ (second row left) is still not sufficient to cover the gold core entirely. The polar chain of $\mathrm{C}_{l}=11$ (second row right) cover the gold core better, because of the repulsive interaction between the polar terminal groups. The longer chains of chain length $\left(\mathrm{C}_{l}\right) 17$ and 23 naturally cover larger part of the gold core but even for the longest studied chains, there are open patches of surface which are accessible by solvent over a time scale of $80 \mathrm{~ns}$. This behavior is not strongly dependent on the polarity of terminal groups. The plots corresponding to the $\mathrm{COO}^{-}$group-terminated systems show similar features as the polar $\mathrm{NH}_{3}^{+}$terminated ones (see in appendix A.4). In summary, water access to the surface is possible for all linear chain coated systems over a time period of $80 \mathrm{~ns}$.

Figure 5.10 shows maps for the GNPs coated $\left(d_{f}=1 / 2\right)$ with branched chains $\left(\mathrm{C}_{l}\right.$ $=17)$. The left column shows maps for nonpolar $\mathrm{CH}_{3}$ group terminated $\left(\mathrm{C}_{l}=17\right)$ branched chains of varying junction points at 0th, 4th, 8th and 12th carbon atom (from top to 

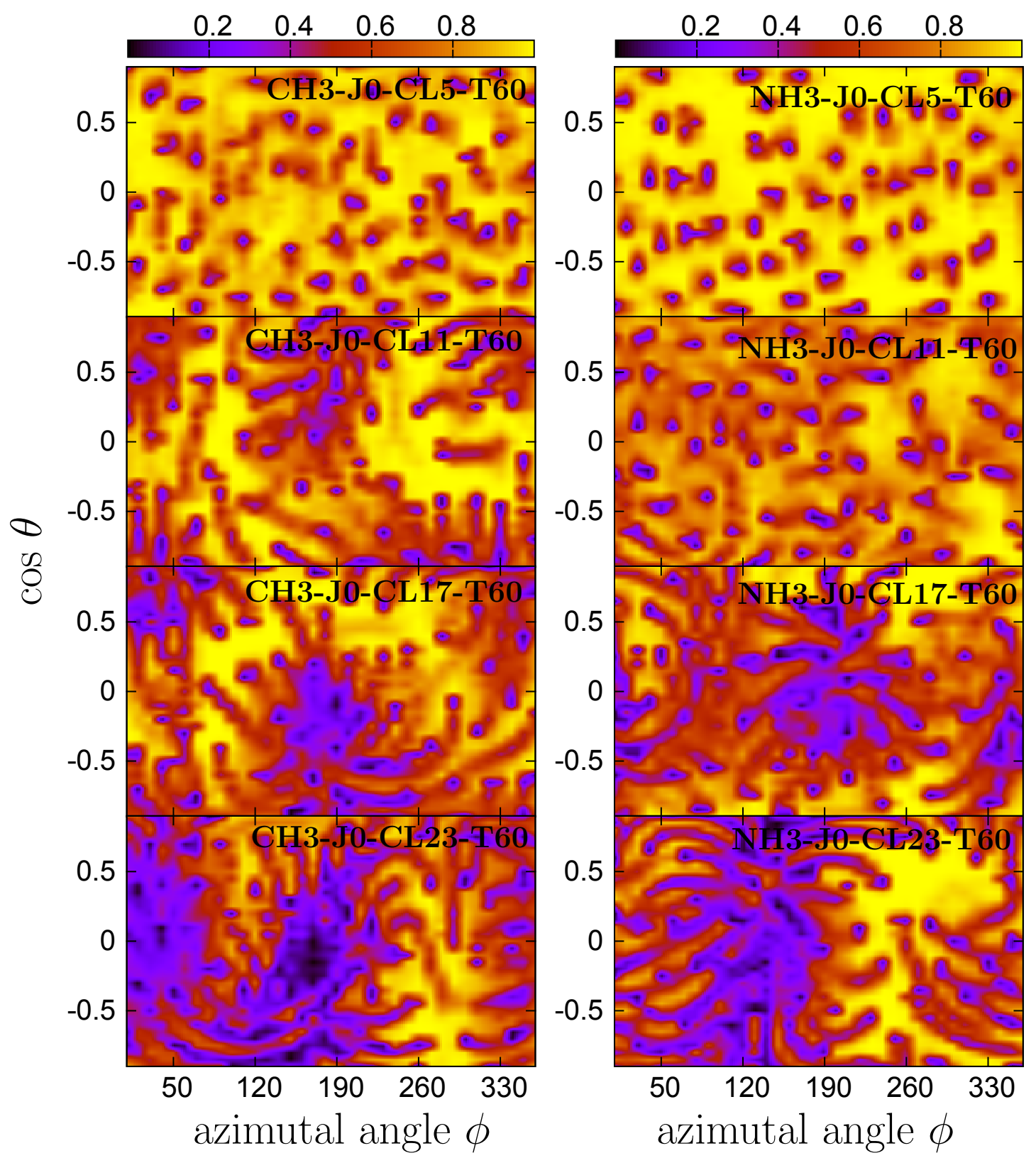

Figure 5.9: Color maps of surface access. Value 1 (yellow) indicates that a oxygen of water molecule is closest to the GNP surface all the time and a value 0 (purple) indicates a chain carbon atom $\left(\mathrm{C}\right.$ of $\mathrm{CH}_{2}$ or $\left.\mathrm{CH}_{3}\right)$ is closest to the surface. Intermediate values correspond to the probability to find water as the closest molecule. Left column: $\mathrm{CH}_{3}$ group terminated linear chains with 5, 11, 17 and 23 carbon atom (from top to bottom). Right column: $\mathrm{NH}_{3}^{+}$group terminated linear chains with 5, 11, 17 and 23 carbon atoms. 
bottom). The right column shows analogous data for $\mathrm{NH}_{3}^{+}$group terminated branched chains $\left(\mathrm{C}_{l}=17\right)$ of varying junction points at 0 th, 4 th, 8th and 12 th carbon atom (from top to bottom).

The maps for branched chains $\left(\mathrm{C}_{l}=17\right)$ with junction at 0 th carbon atoms (top row) have pronounced yellow regions, which indicates that the solvent can access the gold core very easily. The branched chains with the junction at the 4 th carbon atom (second row) mostly cover the gold core, and the nonpolar $\mathrm{CH}_{3}$ group terminated branched chains cover the core better than the polar $\mathrm{NH}_{3}^{+}$group terminated chains. The nonpolar $\mathrm{CH}_{3}$ group terminated chains wrap the core tightly and close all open passage to access the surface, but polar group terminated chains bind the core more loosely due to the repulsive interaction between the terminal groups. The area of yellow regions on the maps start to increase as the chain junction shift from the 4th to the 8th (third row) and the 12th (fourth row) carbon atoms. As the junction position shifts from the surface towards the terminal, chains open the path for solvent to access the surface. This phenomenon is not surprising and it is a consequence of system modeling. To keep the grafting density and the number of terminal groups constant over the gold surface, the number of carbon atoms is continuously reduced with the shift of junction point positions further away, and this leads to easier access of water to the surface for the chains with junction points at higher number (8th and 12th carbon).

Surface access of the branched chain systems with a constant number of carbon atoms have been compared in figure 5.11. Figure 5.11 shows color maps of surface access of branched chain GNPs which have equal numbers of carbon atoms over the surface of the gold cores. The left column shows maps for $\mathrm{CH}_{3}$ group terminated $\left(\mathrm{C}_{l}=17\right)$ branched chains with the junction points at 4 th, 8 th and 12 th carbon atom (top to bottom). The right column shows similar data for $\mathrm{NH}_{3}^{+}$group terminated branched chains $\left(\mathrm{C}_{l}=17\right)$ with the junction points at 4 th, 8 th and 12 th carbon atoms (top to bottom). In the left column, the yellow regions on the maps get larger and larger as the branched chain junction moves from the 4 th to the 8 th and 12 th carbon atoms. Similarly, in the right column the yellow regions get larger with the increase of the distance of the junction points from the gold surface. This shows that chains with a junction at the 4th carbon gives the best protection to the gold core.

The methyl group terminated branched chains of length 17 carbon atoms and branched at the 4th carbon cover the most part of the GNP, but the longest studied 

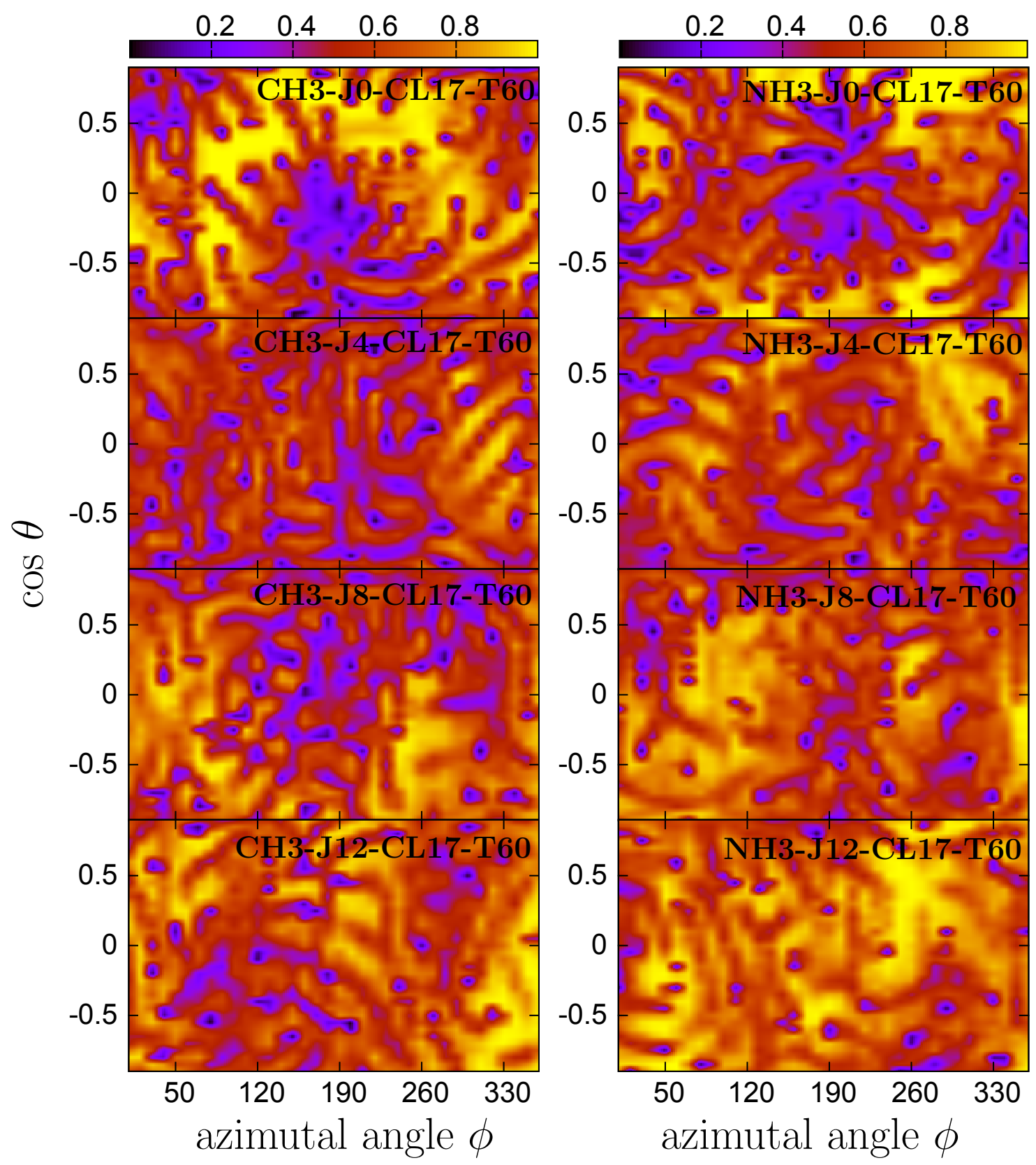

Figure 5.10: Color maps of surface access of GNPs with $\mathrm{C}_{l}=17$. Value 1 (yellow) indicates that a water molecule is closest to the GNP surface and a value 0 (purple) indicates a chain carbon atom is closest to the surface. Left column: $\mathrm{CH}_{3}$ group terminated branched chains with junction at the 0th, 4th, 8th and 12th carbon atom (from top to bottom). Right column: $\mathrm{NH}_{3}^{+}$group terminated branched chains with junction at the 0th, 4th, 8th and 12 th carbon atoms. 

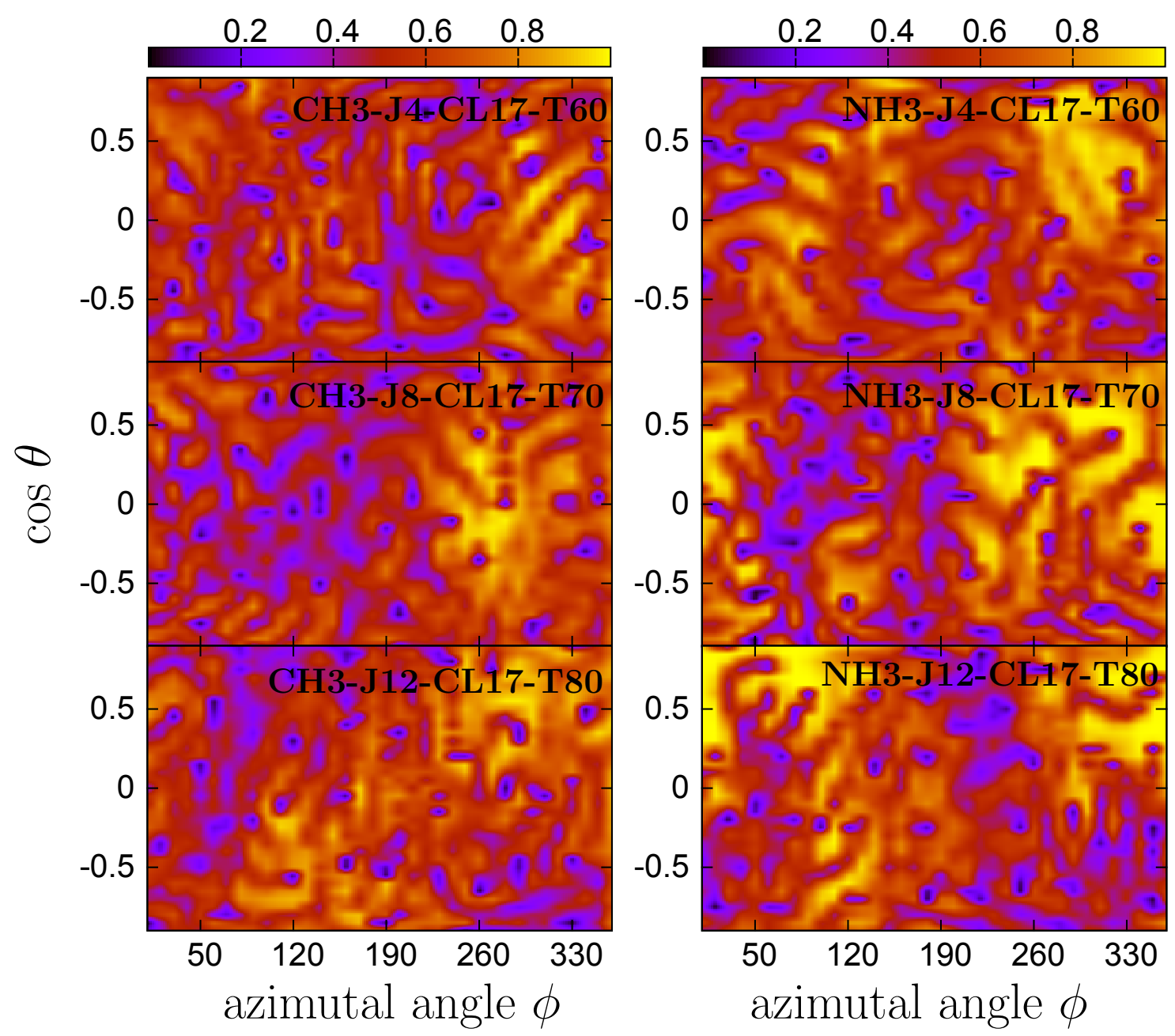

Figure 5.11: Color maps of surface access of GNPs with $\mathrm{C}_{l}=17$ and equal number of carbon atoms on gold cores. Value 1 (yellow) indicates that a water molecule is closest to the GNP surface and a value 0 (purple) indicates a chain carbon atom is closest to the surface. Left column: $\mathrm{CH}_{3}$ group terminated branched chains with junction at the 4th, 8th and 12th carbon atoms (from top to bottom). Right column: $\mathrm{NH}_{3}^{+}$group terminated branched chains with junction at the 4 th, 8 th and 12 th carbon atoms.

linear chains of chain length 23 carbon do not cover the gold core entirely. There are always tiny passages, through which solvent can access the gold core. One can extrapolate that the chains having number of atoms above 25 and branched at 4 th carbon can cover the core entirely and the core would be inaccessible to solvents. Although, too long branched chains (more than 35 carbon atoms) and branched at 4th carbon atom may 
again start to form bundles and can create passages to allow the solvent to reach the core. In summary, water access to the surface is possible for most of the studied systems over time spans of $80 \mathrm{~ns}$, but $\mathrm{CH}_{3}$ group terminated branched chains $\left(\mathrm{C}_{l}=17\right)$ with junction at the 4 th carbon atom provide the best coverage to the core. Corresponding data sets of branched chains with $\mathrm{C}_{l}=11$ show similar features as the branched chains with $\mathrm{C}_{l}=17$, and maps are given in appendix A.5.

\subsubsection{Water Penetration and Shape of GNPs}

Figure 5.12 shows the density plots of water for $\mathrm{CH}_{3}$ group terminated systems. In the left side of the figure, linear chains with chain length 5, 11, 17 and 23, and 11 carbon long branched are compared. In the right side of the figure, 17 carbon long branched chains with equal number of carbon atoms over the surface of gold core, and branched at 4th, 8th and 12 th are compared. Water molecules penetrate up to $11 \AA$ (depth is measured from
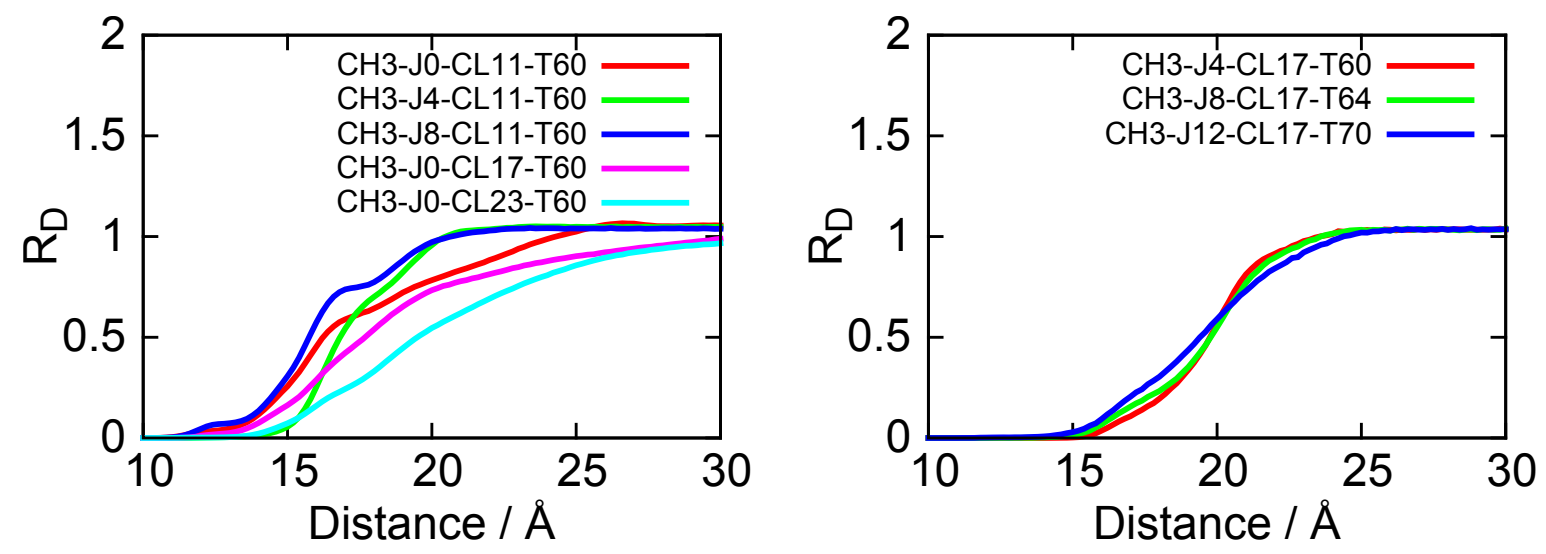

Figure 5.12: Density plots of water for $\mathrm{CH}_{3}$ group terminated systems. Left side: Linear chains with $\mathrm{C}_{l}=5,11,17$ and 23 , and 11 carbon atoms branched chains are compared. Right side: 17 carbon long branched chains with equal number of carbon atoms over the gold surfaces are compared.

the center of gold core) for both the 11 carbon long linear chains (CH3-J0-CL11-T60) and 11 carbon long branched chains with junction at the 8th carbon (CH3-J8-CL11T60). Penetration depth of water for the 11 carbon long branched chain with junction at the 4th carbon (CH3-J4-CL11-T60) stops at $14 \AA$ and thus the penetration depth is lower than the depths for any other 11 carbon long branched chain system (considering 
Table 5.3: Radius of gyration and chain length

\begin{tabular}{|l|l|l|l|l|}
\hline Systems & $\mathrm{R}_{g}(\AA)$ & $\begin{array}{l}\mathrm{R}_{g} \text { Standard } \\
\text { deviation }\end{array}$ & Chain length $(\AA)$ & $\begin{array}{l}\text { Chain length } \\
\text { Standard de- } \\
\text { viation }\end{array}$ \\
\hline CH3-J0-CL11-T60 & 15.93 & 0.13 & 13.08 & 0.19 \\
\hline CH3-J4-CL11-T60 & 14.57 & 0.06 & 10.61 & 0.14 \\
\hline CH3-J8-CL11-T60 & 14.35 & 0.09 & 10.29 & 0.20 \\
\hline CH3-J0-CL17-T60 & 18.72 & 0.14 & 20.08 & 0.31 \\
\hline CH3-J4-CL17-T60 & 16.17 & 0.08 & 15.50 & 0.31 \\
\hline CH3-J8-CL17-T60 & 15.97 & 0.08 & 13.55 & 0.24 \\
\hline CH3-J12-CL17-T60 & 15.50 & 0.14 & 13.84 & 0.21 \\
\hline
\end{tabular}

the depth from the terminal towards center). Penetration of water for the 11,17 and 23 carbon long linear chains stops at $11 \AA, 12 \AA$ and $14 \AA$, respectively. Water penetrates up to $14 \AA$ for both the branched chains of chain length 11 carbon and the linear chain of $\mathrm{C}_{l}=23$. The penetration depth decreases with increasing chain length (considering the depth from the terminal towards center). The water molecules have the same penetration depth for both the short branched chain and the longest linear chain. Penetration depth for 17 carbon long chains with equal number of carbon atoms over the gold surfaces with junctions at 4 th, 8 th and 12 th carbon atoms are very close to each other and penetration depth is lowest for CH3-J4-CL17-T60 among all studied systems (considering the depth from the terminal towards center).

The radius of gyration $\left(\mathrm{R}_{g}\right)$ and chain length of different systems of 12 and 18 carbon long chains are given in table 5.3. The radius of gyration is calculated only for the carbon atoms in the chains. The chain length is calculated taking the distance between the first carbon atom, which is directly connected to a sulfur atom by a bond, and the carbon atom of the $\mathrm{CH}_{3}$ terminal group of the corresponding chain. This chain length is averaged over all chains of a nanoparticle, then averaged over time, to get the final value of chain length.

Radius of gyration data in the table shows that there is a big change in the $\mathrm{R}_{g}$ values when chains are switched from linear to the branched chains. Similar changes also happen for the standard deviations, and the standard deviation values increase with the shift of the junction position further away from the surface. This indicates that the linear chains are stretched away from the gold core and flexible but branched chains are collapsed onto the gold core and are less flexible. This flexibility increases as the junction position moves 
away from the core. The time averaged chain length also shows big changes when chains change from linear to branched. Thus, the time averaged chain lengths are consistent with $\mathrm{R}_{g}$. The $\mathrm{NH}_{3}^{+}$group terminated systems also show similar properties as the $\mathrm{CH}_{3}$ group terminated systems. Values of $\mathrm{R}_{g}$ and time averaged chain length of $\mathrm{NH}_{3}^{+}$terminated systems are given in appendix A.6.

\subsubsection{Solvent Accessible Surface Area}

The solvent accessible surface area (SASA) and the volume occupied by solute in solution (VOSS) are important quantities to understand how the alkyl chains minimize hydrophobic regions. There are several ways to calculate these quantities but the most widely used ways are

1) considering the Voronoi cell ${ }^{105,106}$ for each particle individually, as a polyhedron surrounding the particle,

2) or using the rolling ball algorithm, ${ }^{107}$ which uses a sphere (of solvent) of a particular radius (van der Waals radius) to probe the surface of the solute. In this study technique (1) is used to calculate the quantities. For a set of points in a domain, the Voronoi tessellation is defined by associating a cell of space to each point that is closer to that point than to any other. These cells are created by taking pairs of points that are close to each other and drawing a plane that is equidistant from both of the points and perpendicular to the line connecting the points. To calculate the solvent accessible surface area (SASA) and volume occupied by solute in solution (VOSS), the following steps are taken: ${ }^{105}$

1. The Voronoi tessellation is performed considering all atoms of the GNP (except hydrogen atoms) and the oxygen atoms of water molecules in the system.

2. In the Voronoi diagram, the Voronoi cells corresponding to alkyl chains ("solute cell") and the terminal groups are identified.

3. If the solute cells share any face with neighboring solvent (oxygen) cells, the common surfaces will be added up to get SASA, and the volume of Voronoi cells corresponding to the solute will be summed up to get VOSS.

Figure 5.13 shows the SASA of terminal groups of different systems with chain length 5, 11, 17 and 23 carbon atoms. The SASA of non polar group $\left(\mathrm{CH}_{3}\right)$ is always lower than the SASA of polar groups $\left(\mathrm{NH}_{3}^{+}\right.$and $\left.\mathrm{COO}^{-}\right)$. For short chain systems $\left(\mathrm{C}_{l}=5\right)$ 
the SASA is the same for both the terminals $\mathrm{COO}^{-}$and $\mathrm{CH}_{3}$ but with the increase of chain length, the difference between the SASAs get bigger. The SASA is always higher for $\mathrm{COO}^{-}$terminal than for the $\mathrm{NH}_{3}^{+}$terminal. The SASA for both the polar terminals is more or less constant with the increase of chain length but the SASA of nonpolar terminal group decreases with the increase of chain length.

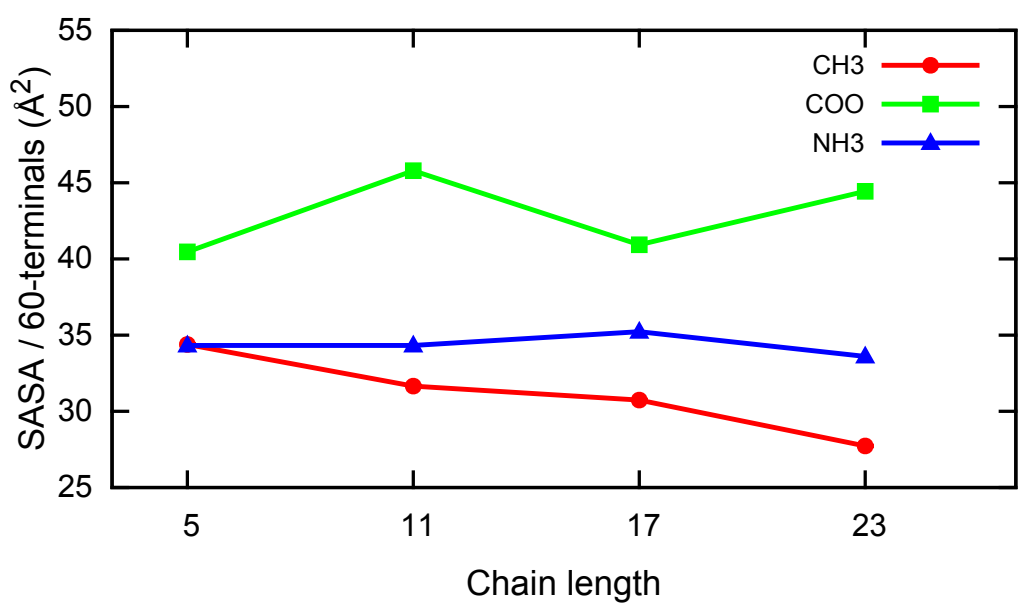

Figure 5.13: SASA of different terminal groups of different systems with chain lengths 5, 11, 17 and 23 carbon atoms. Vertical axis shows SASA per 60 terminal groups of a GNP and horizontal axis shows systems with different chain length.

This indicates that water molecules solvate the $\mathrm{COO}^{-}$terminal group better than the $\mathrm{CH}_{3}$ and $\mathrm{NH}_{3}^{+}$terminals. This helps to form more hydrogen bonds between $\mathrm{COO}^{-}$ terminals and hydrogen atoms of water molecules than between $\mathrm{NH}_{3}^{+}$terminals and oxygen atoms of water molecules (see chapter 4: Hydrogen Bonds). This also indicates that the polar groups are more soluble than the nonpolar group. Solubility of negatively charged terminals $\left(\mathrm{COO}^{-}\right)$is very high irrespective of the chain length. However the difference between the SASA of two polar terminal $\left(\mathrm{COO}^{-}\right.$and $\left.\mathrm{NH}_{3}^{+}\right)$groups arises entirely due to the solvation structures around the terminals.

Figure 5.14 shows the SASA of 23 long carbon chain $\left(\mathrm{C}_{23}\right)$ with different terminal groups. The SASA per four carbon atoms changes along the vertical axis and segments of chain change along the horizontal axis. Counting of chain atoms for this case starts from the surface of the gold core and the carbon atoms which are connected to sulfur atoms directly by a bond are considered as number one. The $\mathrm{C}_{23}$ chains are partitioned into five different segments leaving the last four carbon atoms near the terminals. The last 
four atoms have not taken in consideration because the SASAs for the terminal groups are already discussed. The figure shows that the SASA increases very rapidly from first carbon

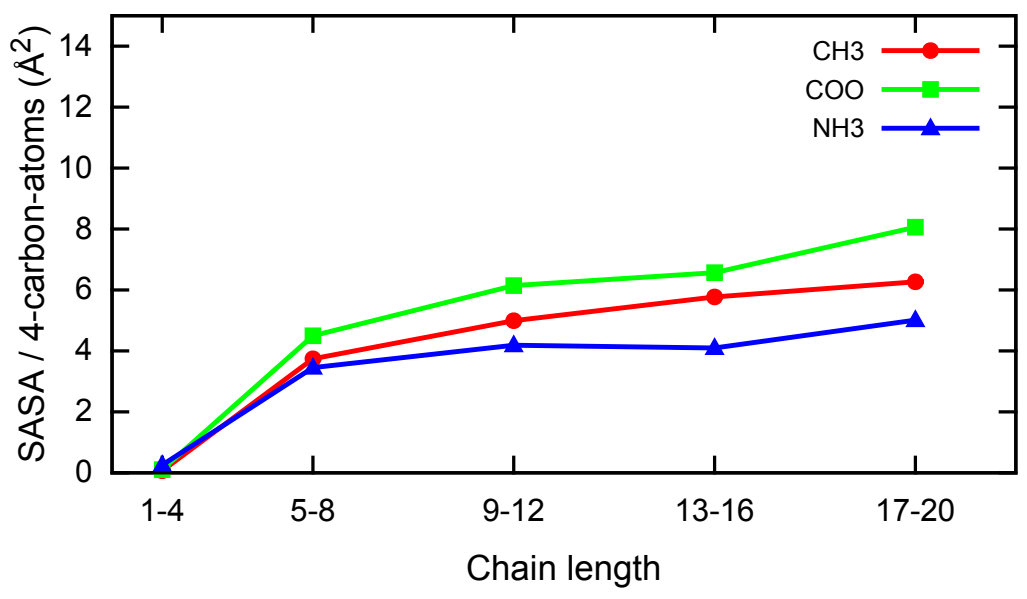

Figure 5.14: SASA of 23 carbon long chains in different segments. SASA per segments of four carbon atoms changes along vertical axis. Segments of chain change along the horizontal axis.

to 8th carbon and then the increment rate becomes smaller. This happens irrespective of the terminal groups. The SASA of $\mathrm{COO}^{-}$group terminated chains is always high in all segments, although the difference between the SASA of different group terminated chains are small (see the vertical scale). This difference between the SASA of different group terminated chains comes from the nature of the bundle formed by the chains. Near the surface of gold cores SASA is close to zero because the density of the chain atoms is very high. As the density of the chain atoms decreases with the distance from the surface, the SASA starts to increase. The increment rate of the SASA becomes slower after a certain distance because the chains try to minimize the hydrophobic region which is exposed to water, which is not possible near the surface due to the high atom density and the constraints (chains are tightly bonded to the gold surface).

Figure 5.15 shows the SASA per six carbon atoms for different systems. Here the SASAs are calculated for $\mathrm{C}_{5}, \mathrm{C}_{11}, \mathrm{C}_{17}$ and $\mathrm{C}_{23}$ systems and then divided by $1,2,3$ and 4 , respectively, to get the SASA per six carbon long segments. This plot helps to get information about the solubility of the different systems. The figure shows that the SASA per segment for the systems with short chain $\left(\mathrm{C}_{l}=5\right)$ are much higher than for the systems with long chain $\left(\mathrm{C}_{l}=23\right)$ and the SASA per segment decreases with the increase 


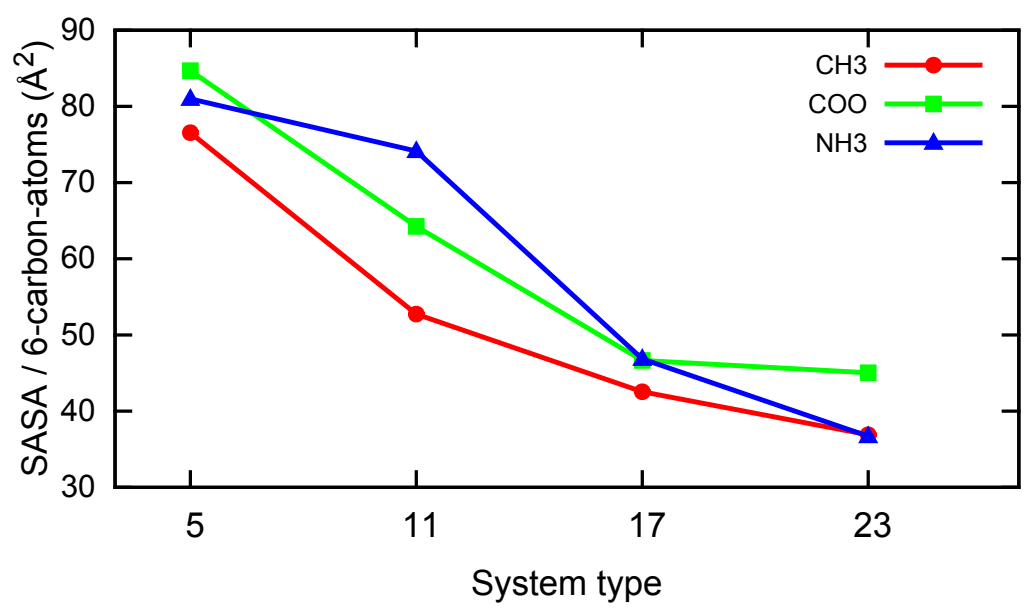

Figure 5.15: SASA per six carbon long segments of different systems. SASA per six carbon atoms segments change along the vertical axis and systems with different chain length change along the horizontal axis.

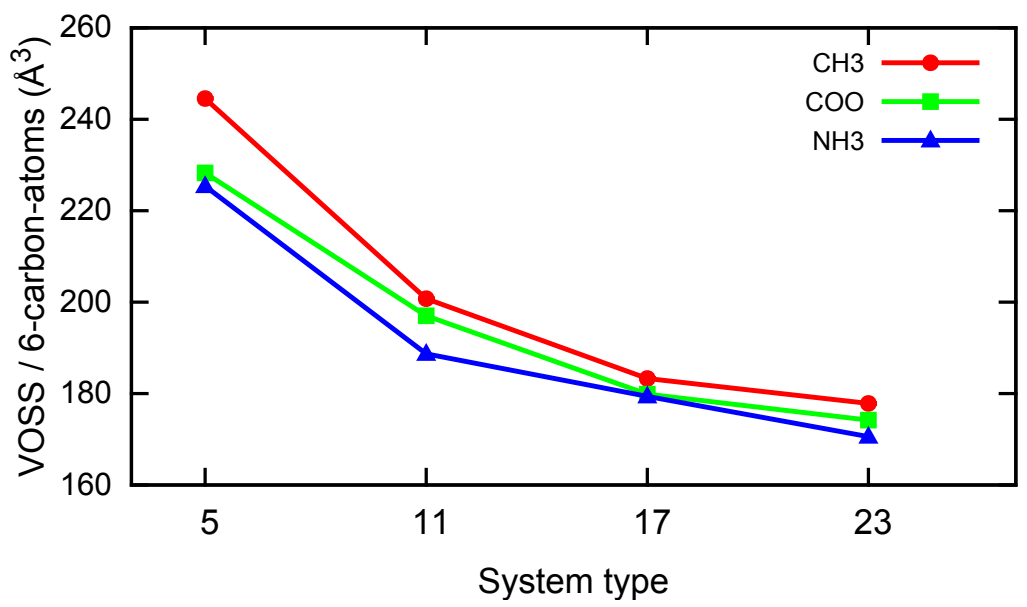

Figure 5.16: VOSS per six carbon long chain of different systems. VOSS per six carbon atoms segment changes along the vertical axis and systems with different chain length change along horizontal axis.

of chain length irrespective of the terminal groups. The short chains are unable to reduce the hydrophobic regions exposed to the water due to the constraint which binds the chains with the gold core. This constraint forces the short chains to distribute uniformly over the gold core. This uniform distribution helps the water molecule to solvate the chains easily. As the terminals are free to move, so the chain segments (away from the surface) 
of long chains try to minimize the hydrophobic regions exposed to water and this lead to low SASAs for long chain systems. Not only the constraints but also the polar terminal groups prohibit the chains to minimize the hydrophobic regions which is exposed to water, and this makes big differences between SASAs for the chain lengths $\mathrm{C}_{l}=11$.

Figure 5.16 shows the VOSS per per six carbon atoms long chain of different systems. The VOSS per six carbon atoms segments changes along the vertical axis and systems with different chain length change along the horizontal axis. The figure show that the VOSS per segment decreases with the increase of the chain length. The short chains are uniformly distributed on the gold surface and this leads to high VOSS per segment for the short chains but long chains form bundles which reduce the VOSS per segment for long chains. In summary, the GNP with $\mathrm{COO}^{-}$group terminated chains of chain length $\mathrm{C}_{l}$ $=5$ should be highly soluble and on the other hand the GNP with $\mathrm{CH}_{3}$ group terminated chains of chain length $\mathrm{C}_{l}=23$ can be expected to be the least soluble. 
5.2. Results and Discussion 


\section{Conclusions}

Computer simulations of ionic bulk solutions, and of functionalized gold nanoparticles in aqueous solution were performed and analyzed in this thesis. The results of the simulated systems were discussed in three different parts.

In the first part (chapter 3), the results of bulk $\mathrm{NaCl}$ solution systems were described. For simulations of bulk $\mathrm{NaCl}$ solutions, three different force field parameter sets, namely AMBER-99, CHARMM-27 and OPLS-AA, and two combination rules, namely geometric and arithmetic, were used. 15 different systems with different solution concentrations $(1.1 \mathrm{~m}, 1.5 \mathrm{~m}$ and $2.0 \mathrm{~m})$, force field parameter sets and combination rules were compared to identify a suitable set of force field parameters and combination rules. All systems were simulated for 20 ns. The systems with AMBER-99 and arithmetic combination rules show indication of crystallization within 20 ns simulation time. This crystallization process is slow at low concentration, and its rate increases with increasing concentration of the solution. The systems with AMBER-99 and geometric combination rules do not show any indication of crystal formation at low concentrations, but at high concentration $(2.0 \mathrm{~m})$ the system shows crystallization. Systems with OPLS-AA and arithmetic combination rules show crystallization at high concentrations $(1.5 \mathrm{~m}$ and $2.0 \mathrm{~m})$, but systems with OPLS-AA and geometric combination rules do not show any crystallization even at high concentration $(2.0 \mathrm{~m})$. However, the systems with CHARMM-27 and arithmetic combination rule do not show crystallization even at the higher concentration $(2.0 \mathrm{~m})$.

The unphysical behavior (crystal formation at low concentration) of the systems with AMBER-99 is a consequence of shortcomings in the parametrization of ions in AMBER99. AMBER-99 adopted cation parameters directly from Åvist, who used geometric combination rules for ion pairs. Although the situation improves for the systems with AMBER-99 and geometric combination rules, AMBER-99 is nevertheless not suitable for the simulation of $\mathrm{NaCl}$ solutions with concentration higher than 1 molal. The OPLS-AA 
with geometric combination rules and CHARMM-27 with arithmetic combination rules do not show any unrealistic behavior. Thus, OPLS-AA with geometric combination rules and CHARMM-27 with arithmetic combination rules are suitable for the simulation of $\mathrm{NaCl}$ solution system even at high concentration ( 2 molal). The popular force field parameter set AMBER-99 with arithmetic combination rule can be used for the $\mathrm{NaCl}$ system by replacing the ion parameters of AMBER-99 by the CHARMM-27 ion parameters. For the simulations of GNP systems (chapter 4), AMBER-99 with the replacement of ion parameters from CHARMM-27, and arithmetic combination rules have been used. For the simulations of GNP systems in chapter 5, OPLS-AA and geometric combination rules have been used.

In chapter 4, small GNPs of approximately $2 \mathrm{~nm}$ diameter, functionalized with $C_{l}=11$ hydrocarbon chains and terminated with nonpolar $\mathrm{CH}_{3}$ and polar $\mathrm{COO}^{-}$and $\mathrm{NH}_{3}^{+}$groups have been studied. The GNPs were solvated in 1 molar NaCl solution and the charge of the polar group terminated GNPs was compensated by excess $\mathrm{Na}^{+}$or $\mathrm{Cl}^{-}$ counterions. For each type of terminal group, systems with degrees of functionalization $d_{f}=1,2 / 3$ and $1 / 3$ were compared. At high $d_{f}$, the end-to-end distances of different group terminated chains are more or less equal, and at low $d_{f}$ all chains collapse to some extent on the gold core. The polar group terminated chains at low $d_{f}$ have longer effective chain length (end-to-end distance) than nonpolar group terminated chains. Overall, the nonpolar group terminated chains wrap the gold core better than the corresponding polar group terminated chains. Even so, at degrees of functionalization $d_{f}=1$ parts of the gold core are still accessible to water, irrespective of the terminal group. The counter ions around the polar terminals screen the charged terminal groups, and this effect leads to a significant slowdown in motion of counter ions in the head group region which, in turn act back on chain conformation and dynamics. The terminal dihedrals of polar terminals show more static disorder than the corresponding methyl group terminated chains and this disorder increases with grafting density. Simultaneously, the terminal dihedral angles of polar chains show significantly longer relaxation times than the corresponding ones of nonpolar chains. The solvation shell structures around the positively and negatively charged terminals are different, which influences the hydrogen bonds and contact ion distribution around the polar terminals. The numbers of hydrogen bonds and of contact ion per $\mathrm{COO}^{-}$terminal group are higher than the corresponding numbers per $\mathrm{NH}_{3}^{+}$ terminal group. The number of hydrogen bonds per terminal group decreases with the increase of grafting density, but the number of contact ions per terminal group increases 
with the increase of grafting density. The total integrated charge close to the gold core is always positive, which is entirely due to water orientation, not to ion penetration. Water molecules orient near the surface of the gold core pointing one hydrogen atom towards the center of the gold core irrespective of the system. Also, the orientation of water molecules near the polar terminal groups is the reason for the positive effective total integrated charge near the terminal. In polar chain systems, the effective dipole of water changes its direction depending on the distance from the gold surface and this orientation of the dipole is influenced by the physical constraints, hydrophobicity and the terminal charge. The influence of functionalized GNPs $\left(\mathrm{C}_{l}=11\right.$ and $\left.d_{f}=1\right)$ on the solution structure last up to about $2.8 \mathrm{~nm}$ (maximum) from the center of the gold core.

The results of the simulations of functionalized gold nanoparticles show the influence of the aqueous solution on the particles. It can be seen from the results that such small and fully functionalized gold nanoparticles do not prevent water access to the gold core at a time scale of $40 \mathrm{~ns}$ and beyond. Thus, non-noble nanoparticles, whose cores consist of acid-base instable or water-soluble or oxidizable metals can be expected to be readily attacked by the aqueous solution, even though the degree of functionalization is very high. This problem can be solved by increasing the grafting density to an even higher value than the studied densities. If the increase of grafting density is undesirable or not possible, then a possible remedy for the stabilisation of such particles in aqueous environment would be the use of branched chains on the metal core. This topic forms the third part of this thesis.

In chapter 5 , small GNPs (like in chapter 4), functionalized with nonpolar $\mathrm{CH}_{3}$ and polar $\mathrm{COO}^{-}$and $\mathrm{NH}_{3}^{+}$group terminated linear and branched chains of various chain lengths have been studied. A total of 26 systems were modeled by changing the chain length $\left(C_{l}=5,11,17\right.$ and 23), terminal groups $\left(\mathrm{CH}_{3}, \mathrm{COO}^{-}\right.$and $\left.\mathrm{NH}_{3}^{+}\right)$, the positions of the junction point of the branched chain (4th, 8th and 12th carbon) and the grafting density. The linear chain grafted gold nanoparticles show coating asymmetry, although the chains are uniformly grafted on the gold core. This coating asymmetry arises due to the bundle formation of chains discussed above. Short chains $\left(C_{l}=5\right)$ are uniformly distributed and do not show any coating asymmetry, but chains of length $C_{l}=11$ show mixed behavior, depending on the terminal groups. Nonpolar $\left(\mathrm{CH}_{3}\right)$ terminated chains of chain length $C_{l}=11$ show coating asymmetry, but polar group $\left(\mathrm{COO}^{-}\right.$and $\left.\mathrm{NH}_{3}^{+}\right)$terminated chains do not show coating asymmetry. Electrostatic interactions between the polar terminals prohibit the chains $\left(C_{l}=11\right)$ to form bundles, which leads to relatively symmetric 
coating of the gold core. However, this interaction can not prohibit coating asymmetry for long chains of chain length $C_{l}=17$ and 23, irrespective of their terminal groups. Bundle formation of long linear chains does not start right at the gold surface, rather it starts after the 7th carbon atom (approximately) of a chain (taking the carbon atom which is directly connected to a sulfur via a bond as number one). The bundle formation of chains arises as a consequence of multiple effects such as solvent-ligand interaction, hydrophobicity, geometric constants, ligand-ligand interaction, grafting density, curvature of the gold core, shape of the gold core (which was not varied in this study) and free volume available to the chains. Near the surface of the gold core, the hydrophobic region of one chain is in touch with another chain but at longer distance (after the 7th carbon atom), the free volume is larger than the total volume of the chains. Thus, at long distances from the surface, uniform distribution of chains would expose the hydrophobic regions to water, and thus to overcome this unfavorable situation, the chains form bundles to reduce the hydrophobic regions exposed to water. This bundle formation does not happen for branched chains, irrespective of the terminal group. All branched chains irrespective of their chain length and junction position thus prevent a strongly asymmetric distribution of chains. Branched chains have physical constraints which form a certain angle near the junction between two chains and this prevents the chains to come close enough to form bundles. Instead of bundle formation, branched chains wrap the core more tightly in order to minimize the contact area between hydrophobic region of chains and water. Water can access the gold surface even for the longest studied linear chain of length $C_{l}=23$, and this is partly a consequence of the bundle formation, which allows relatively easy passage of water to the surface of the gold core. Hence, mere increase of chain length does not help to entirely prevent water access to the core. Branched chains of length $C_{l}=17$ and branched at 4 th carbon however prevent most water molecules to access the surface. The analysis shows that the $\mathrm{CH}_{3}$ group terminated branched chains of length $C_{l}=17$ provide the best protection to the gold core. The solubility of the functionalized GNPs can be estimated by measuring the solvent accessible surface area (SASA). The SASA of functionalized nanoparticles is highly dependent on chain lengths and terminal groups. It decreases with increasing linear chain length (also per unit length). Among all studied linear chain GNPs, the SASA (per unit chain length) of the GNP coated with $\mathrm{COO}^{-}$ terminated chains of length $C_{l}=5$ has the highest value, and the SASA (per unit chain length) of a GNP coated with $\mathrm{CH}_{3}$ group terminated linear chains of chain length $\mathrm{C}_{l}=23$ has the lowest value. Hence, the GNP coated with $\mathrm{COO}^{-}$group terminated linear chains of chain length $\mathrm{C}_{l}=5$ should be highly soluble, and the GNPs coated with $\mathrm{CH}_{3}$ group 
terminated linear chains of chain length $\mathrm{C}_{l}=23$ can be expected to be the least soluble.

The results show that coating asymmetry can be prevented almost completely by introducing branched chains instead of long linear chains. Branched chains not only prevent the asymmetry in coating, but also prevent water molecules to access the gold surface more thoroughly than the corresponding linear chains. Nonpolar group terminated chains branched at the 4th carbon provide the best protection to the core in the class of molecules with the same chain length. Perfect protection to the core can be achieved by employing $\mathrm{CH}_{3}$ group terminated branched chains of length $C_{l}>25$ and branched at 4 th carbon $\left(d_{f}=1\right)$. 


\section{List of Figures}

2.1 Sketch of bond stretching, angle bending and torsional rotation . . . . . 10

2.2 Sketch of TIP3P water model with partial charges and angle . . . . . . . . 20

2.3 Sketch of RDF. Atoms are highlighted in side a shell . . . . . . . . . . . 21

2.4 Sketch of hydrogen bonds between water molecule, and $\mathrm{COO}^{-}$and $\mathrm{NH}_{3}^{+}$ terminals . . . . . . . . . . . . . . . . . . . . . . . 24

3.1 Snapshots of solution system. AMBER-99 force parameters are used for the simulations . . . . . . . . . . . . . . . . . . . . . . . . . . . . . . . . . 29

3.2 RDFs of different solution systems with different concentrations. AMBER99 force field parameters are used . . . . . . . . . . . . . . . 30

3.3 $\mathrm{Na}-\mathrm{Cl}$ coordination number of different systems with different concentrations. OPLS-AA force field parameters are used . . . . . . . . . . . . . 32

$3.4 \mathrm{Na}-\mathrm{Cl}$ coordination numbers of different systems with different concentrations. CHARMM-27 force field parameters are used . . . . . . . . . . . . . 33

4.1 Snapshots of different shells of the gold core . . . . . . . . . . . . . 36

4.2 Snapshot of the gold core with sulfur-oxygen bridge . . . . . . . . . . . 37

4.3 Snapshot of a linear chain functionalized gold nanoparticle (LCFGNP) with $60-\left(\mathrm{CH}_{2}\right)_{11} \mathrm{COO}^{-}$in solution . . . . . . . . . . . . . . . 38

4.4 Snapshots of fully and partially functionalized gold nanoparticles with different terminal groups . . . . . . . . . . . . . . . . . 40 
4.5 Time averaged chain length of hydrocarbon chains and radius of gyration of all systems . . . . . . . . . . . . . . . . . . . . . . . 41

4.6 Radial density distributions around the center of gold nanoparticles for different systems ....................... . . 43

4.7 Color maps of surface access for $\mathrm{CH}_{3}$ and $\mathrm{COO}^{-}$terminated systems with different grafting densities . . . . . . . . . . . . . . . . . 45

4.8 Solvation number distribution for $\mathrm{COO}^{-}$and $\mathrm{NH}_{3}^{+}$terminated systems with different grafting densities . . . . . . . . . . . . . . . . 47

4.9 Fraction of trans conformations for individual dihedral angles in $\mathrm{NH}_{3}^{+}$, $\mathrm{COO}^{-}$and $\mathrm{CH}_{3}$ group terminated systems . . . . . . . . . . . . . . . 49

4.10 Relaxation time of individual torsional angle for all fully and partially functionalized systems . . . . . . . . . . . . . . . . . . . 50

4.11 Ion self diffusion coefficients for $\mathrm{CH}_{3}, \mathrm{COO}^{-}$and $\mathrm{NH}_{3}^{+}$group terminated systems . . . . . . . . . . . . . . . . . . 52

4.12 Hydrogen bonds and contact ions per polar terminal groups for different GNP systems ........................ 54

4.13 Radially integrated charge of fully covered GNP systems. individual contributions and total contribution . . . . . . . . . . . . . 55

4.14 Sketch of dipole vector, $\mathrm{H}-\mathrm{H}$ vector, $\mathrm{O}-\mathrm{H}$ vector, normal vector, and the vector connecting the center of gold core and the oxygen of the water . . . 56

4.15 Distribution of angle of dipole vector and $\mathrm{O}-\mathrm{H}$ vector . . . . . . . . . . 57

4.16 Distribution of angle of normal vector and $\mathrm{H}-\mathrm{H}$ vector . . . . . . . . . . . 59

4.17 Sketch of water orientation in different shells from the surface of the gold core for CH3-60 system. . . . . . . . . . . . . . . . . . 60

4.18 Sketch of water orientation in different shells from the surface of the gold core for COO-60 system. . . . . . . . . . . . . . . 61

4.19 Sketch of water orientation in different shells from the surface of the gold core for NH3-60 system. . . . . . . . . . . . . . . . . . 62 
5.1 Snapshot of Fully functionalized GNP with $60-\left(\mathrm{CH}_{2}\right)_{11}-\mathrm{NH}_{3}^{+}$chains . . . . 66

5.2 Schematic representation of $\mathrm{NH}_{3}^{+}$terminated branched chains with $\mathrm{C}_{l}=12 \quad 67$

5.3 Snapshots of linear chain functionalized gold nanoparticles . . . . . . . . . 72

5.4 Schematic representation of angle for time average angle distribution . . . 73

5.5 Time averaged angle distribution of $\mathrm{CH}_{3}, \mathrm{COO}^{-}$and $\mathrm{NH}_{3}^{+}$groups terminated systems ............................ 74

5.6 Snapshots of branched chain coated gold nanoparticles with $\mathrm{C}_{l}=11 \ldots 76$

5.7 Snapshots of branched chain coated gold nanoparticles with $\mathrm{C}_{l}=17 \ldots 77$

5.8 Time averaged angle distribution of $\mathrm{CH}_{3}$ and $\mathrm{NH}_{3}^{+}$groups terminated branched chain systems . . . . . . . . . . . . . . . 79

5.9 Color maps of surface access of different linear chained GNP systems . . 81

5.10 Color maps of surface access of different branched chain GNP . . . . . . . 83

5.11 Color maps of surface access of GNPs with $\mathrm{C}_{l}=17$ and equal number of carbon atoms on gold cores . . . . . . . . . . . . . 84

5.12 Density plots of water for $\mathrm{CH}_{3}$ group terminated systems . . . . . . . . 85

5.13 SASA of different terminal groups of different systems . . . . . . . . . 88

5.14 SASA of 23 carbon long chains in different segments . . . . . . . . . . 89

5.15 SASA per six carbon long chain of different systems . . . . . . . . . . 90

5.16 VOSS per six carbon long segments of different systems . . . . . . . . . 90

A.1 Color maps of surface access for $\mathrm{NH}_{3}^{+}$terminated systems with different grafting densities . . . . . . . . . . . . . . . 120

A.2 Time averaged angle distribution of the systems of chain length 5 and 17 . 121

A.3 Color maps of surface access for $\mathrm{NH}_{3}^{+}$terminated systems with different chain length . . . . . . . . . . . . . . . . . . 122

A.4 Color maps of surface access for the systems of branched chain length 11 . 123 
List of Figures 


\section{List of Tables}

3.1 Force field parameters of ions $\ldots \ldots \ldots \ldots \ldots$

3.2 Comparison of force fields $\ldots \ldots \ldots \ldots 33$

4.1 AMBER-99 and CHARMM-27 force field parameters . . . . . . . . . 39

5.1 Summary of system description $\ldots \ldots \ldots$. . . . . . . . . . . . 69

5.2 OPLS-AA force field parameters $\ldots \ldots \ldots \ldots \ldots$

5.3 Radius of gyration and chain length . . . . . . . . . . . 86

A.1 Radius of gyration and chain length of $\mathrm{NH}_{3}^{+}$terminated chains . . . . . . 124 
List of Tables 


\section{References}

[1] C. P. Collier, R. J. Saykally, J. J. Shiang, S. E. Henrichs, and J. R. Heath. Reversible tuning of silver quantum dot monolayers through the metal-insulator transition. Science, 277:1978-1981, 1997.

[2] G. Schmid and U. Simon. Gold nanoparticles: Assembly and electrical properties in 13 dimensions. Chem. Commun., 6:697-710, 2005.

[3] G. Schmid. The relevance of shape and size of au55 clusters. chem. soc. rev., 37:1909-1930, 2008.

[4] Michelle Duval Malinsky, K. Lance Kelly, George C. Schatz, and Richard P. Van Duyne. Chain length dependence and sensing capabilities of the localized surface plasmon resonance of silver nanoparticles chemically modified with alkanethiol selfassembled monolayers. J. Am. Chem. Soc, 123:1471-1482, 2001.

[5] K. George Thomas and Prashant V. Kamat. Chromophore-functionalized gold nanoparticles. Acc. Chem. Res, 36:888-898, 2003.

[6] Richard R. Durand Jr., C. Susana Bencosme, James P. Collman, and Fred C. Anson. Mechanistic aspects of the catalytic reduction of dioxygen by cofacial metalloporphyrins. J. Am. Chem. Soc, 105:2710-2718, 1983.

[7] Michael Chandross, Gary S. Grest, and Mark J. Stevens. Friction between alkylsilane monolayers: molecular simulation of ordered monolayers. Langmuir, 18:83928399, 2002.

[8] Krishnendu Saha, Sarit S. Agasti, Chaekyu Kim, Xiaoning Li, and Vincent M. Rotello. Gold nanoparticles in chemical and biological sensing. Chem. Rev, 112:2739-2779, 2012. 
[9] Chang-Cheng You, Oscar R. Miranda, Basar Gider, Partha S. Ghosh, Ik-Bum Kim, Belma Erdogan, Sai Archana Krovi, Uwe H. F. Bunz, and Vincent M. Rotello. Detection and identification of proteins using nanoparticle-fluorescent polymer 'chemical nose' sensors. Nat. Nanotechnol., 2:318-323, 2007.

[10] Oscar R Miranda, Brian Creran, and Vincent M Rotello. Array-based sensing with nanoparticles: chemical noses for sensing biomolecules and cell surfaces. Curr. Opin. Chem. Biol., 14:728-736, 2010.

[11] Shan Jiang, Khin Yin Win, Shuhua Liu, Choon Peng Teng, Yuangang Zheng, and Ming-Yong Han. Surface-functionalized nanoparticles for biosensing and imagingguided therapeutics. Nanoscale, 5:3127-3148, 2013.

[12] Heebeom Koo, Myung Sook Huh, Ju Hee Ryu, Dong-Eun Lee, In-Cheol Sun, Kuiwon Choi, Kwangmeyung Kim, and Ick Chan Kwon. Nanoprobes for biomedical imaging in living systems. Nanotoday, 6:204-220, 2011.

[13] Yen-Chun Shiang, Chih-Ching Huang, Wei-Yu Chen, Po-Cheng Chen, and HuanTsung Chang. Fluorescent gold and silver nanoclusters for the analysis of biopolymers and cell imaging. J. Mater. Chem., 22:12972-12982, 2012.

[14] Tennyson L. Doane and Clemens Burda. The unique role of nanoparticles in nanomedicine: Imaging, drug delivery and therapy. Chem. Soc. Rev., 41:2885-2911, 2012.

[15] Tennyson Doane and Clemens Burda. Nanoparticle mediated non-covalent drug delivery. Adv. Drug Delivery Rev., 65:607-621, 2013.

[16] Bradley Duncan, Chaekyu Kim, and Vincent M. Rotello. Gold nanoparticle platforms as drug and biomacromolecule delivery systems. J. Controlled Release, 148:122-127, 2010.

[17] Eugene Mahon, Anna Salvati, Francesca Baldelli Bombelli, Iseult Lynch, and Kenneth A. Dawson. Designing the nanoparticle-biomolecule interface for "targeting and therapeutic delivery". J. Controlled Release, 161:164-174, 2012.

[18] Christian Argyo, Veronika Weiss, Christoph Bruchle, and Thomas Bein. Multifunctional mesoporous silica nanoparticles as a universal platform for drug delivery. Chem. Mater., 26:435-451, 2014. 
[19] Avelino Corma and Hermenegildo Garcia. Supported gold nanoparticles as catalysts for organic reactions. Chem. Soc. Rev., 37:2096-2126, 2008.

[20] Cristina Della Pina, Ermelinda Falletta, Laura Prati, and Michele Rossi. Selective oxidation using gold. Chem. Soc. Rev., 37:2077-2095, 2008.

[21] Nicholas L. Abbott, John P. Folkers, and George M. Whitesides. Manipulation of the wettability of surfaces on the 0.1 - to 1 -micrometer scale through micromachining and molecular self-assembly. Science, 257:1380-1382, 1992.

[22] C. Daniel Frisbie, John R. Martin, Jr. Ronald R. Duff, and Mark S. Wrighton. Use of high lateral resolution secondary-ion mass spectrometry to characterize selfassembled monolayers on microfabricated structures. J. Am. Chem. Soc, 114:7142$7145,1992$.

[23] Marie-Christine Daniel and Didier Astruc. Gold nanoparticles: assembly, supramolecular chemistry, quantum-size-related properties, and applications toward biology, catalysis, and nanotechnology. Chem. Rev., 104:293-346, 2004.

[24] Royce W. Murray. Nanoelectrochemistry: Metal nanoparticles, nanoelectrodes, and nanopores. Chem. Rev., 108:2688-2720, 2008.

[25] Michael Walter, Jaakko Akola, Olga Lopez-Acevedo, Pablo D. Jadzinsky, Guillermo Calero, Christopher J. Ackerson, Robert L. Whetten, Henrik Grönbeck, and Hannu Häkkinen. A unified view of ligand-protected gold clusters as superatom complexes. Natl. Acad. Sci., 105:9157-9162, 2008.

[26] Olga Lopez-Acevedo, Katarzyna A. Kacprzak, Jaakko Akola, and Hannu Hkkinen. Quantum size effects in ambient co oxidation catalysed by ligand-protected gold clusters. Nat. Chem., 2:329-334, 2010.

[27] Dan S. Bolintineanu, J. Matthew D. Lane, and Gary S. Grest. Effects of functional groups and ionization on the structure of alkanethiol-coated gold nanoparticles. Langmuir, 30:11075-11085, 2014.

[28] Tsai-Hua Chung, Si-Han Wu, Ming Yao, Chen-Wen Lu, Yu-Shen Lin, Yann Hung, Chung-Yuan Mou, Yao-Chang Chen, and Dong-Ming Huang. The effect of surface charge on the uptake and biological function of mesoporous silica nanoparticles in 3t3-11 cells and human mesenchymal stem cells. Biomaterials, 28:2959-2966, 2007. 
[29] P. V. AshaRani, Grace Low Kah Mun, Manoor Prakash Hande, and Suresh Valiyaveettil. Cytotoxicity and genotoxicity of silver nanoparticles in human cells. ACS Nano, 3:279-290, 2009.

[30] Yu Zhang, Mo Yang, Ji-Ho Park, Jennifer Singelyn, Huiqing Ma, Michael J. Sailor, Erkki Ruoslahti, Mihrimah Ozkan, and Cengiz Ozkan. A surface-charge study on cellular-uptake behavior of f3-peptide-conjugated iron oxide nanoparticles. Small, 5:1990-1996, 2009.

[31] Cheng-An J. Lin, Ting-Ya Yang, Chih-Hsien Lee, Sherry H. Huang, Ralph A. Sperling, Marco Zanella, Jimmy K. Li, Ji-Lin Shen, Hsueh-Hsiao Wang, Hung-I Yeh, Wolfgang J. Parak, and Walter H. Chang. Synthesis, characterization, and bioconjugation of fluorescent gold nanoclusters toward biological labeling applications. ACS Nano, 3:395-401, 2009.

[32] Christopher J. Ackerson, Pablo D. Jadzinsky, Jonathan Z. Sexton, David A. Bushnell, and Roger D. Kornberg. Synthesis and bioconjugation of 2 and $3 \mathrm{~nm}$-diameter gold nanoparticles. Bioconjugate Chem., 21:214-218, 2010.

[33] Mary-Catherine Bowman, T. Eric Ballard, Christopher J. Ackerson, Daniel L. Feldheim, David M. Margolis, and Christian Melander. Inhibition of hiv fusion with multivalent gold nanoparticles. J. Am. Chem. SOC, 130:6896-6897, 2008.

[34] Steven D. Perrault and Warren C. W. Chan. In vivo assembly of nanoparticle components to improve targeted cancer imaging. Proc. Nat. Acad. Sci. USA, 107:1119411199, 2010.

[35] Bumjoon J. Kim, Joona Bang, Craig J. Hawker, and Edward J. Kramer. Effect of areal chain density on the location of polymer-modified gold nanoparticles in a block copolymer template. Macromol., 39:4108, 2006.

[36] Pradip Kr. Ghorai and Sharon C. Glotzer. Molecular dynamics simulation study of self-assembled monolayers of alkanethiol surfactants on spherical gold nanoparticles. J. Phys Chem. C, 111:15857-15862, 2007.

[37] W. D. Luedtke and U. Landman. Structure and thermodynamics of self-assembled monolayers on gold nanocrystallites. J. Phys. Chem. B, 102:6566-6572, 1998. 
[38] J. M. D. Lane and G. S. Grest. Spontaneous asymmetry of coated spherical nanoparticles in solution and at liquid-vapor interfaces. Phys. Rev. Lett., 104:235501-235504, 2010 .

[39] Zhen Yang, Yunzhi Li, Guobing Zhou, Xiangshu Chen, Duanjian Tao, and Na Hu. Molecular dynamics simulations of hydrogen bond dynamics and far-infrared spectra of hydration water molecules around the mixed monolayer-protected au nanoparticle. J. Phys Chem. C, 119:1768-1781, 2015.

[40] An-Cheng Yang and Cheng-I Weng. Structural and dynamic properties of water near monolayer-protected gold clusters with various alkanethiol tail groups. J. Phys Chem. C, 114:8697-8709, 2010.

[41] Peijun Guo, Rastko Sknepnek, and Monica Olvera de la Cruz. Electrostatic-driven ridge formation on nanoparticles coated with charged end-group ligands. J. Phys Chem. C, 115:6484-6490, 2011.

[42] E. Heikkilä, A. A. Gurtovenko, H. Martinez-Seara, H. Häkkinen, I. Vattulainen, and J. Akola. Atomistic simulations of functional $\mathrm{au}_{144}(\mathrm{sr})_{60}$ gold nanoparticles in aqueous environment. J. Phys Chem. C, 116:9805-9815, 2012.

[43] Elena Heikkila, Hector Martinez-Seara, Andrey A. Gurtovenko, Matti Javanainen, Hannu Häkkinen, Ilpo Vattulainen, and Jaakko Akola. Cationic Au nanoparticle binding with plasma membrane-like lipid bilayers: Potential mechanism for spontaneous permeation to cells revealed by atomistic simulations. J. Phys Chem. C, 118:11131-11141, 2014.

[44] Reid C. Van Lehn and Alfredo Alexander-Katz. Ligand-mediated short-range attraction drives aggregation of charged mono layer-protected gold nanoparticles. Langmuir, 29:8788-8798, 2013.

[45] Jia-Qi Lin, Hong-Wu Zhang, Zhen Chen, Yong-Gang Zheng, Zhong-Qiang Zhang, and Hong-Fei Ye. Simulation study of aggregations of monolayer-protected gold nanoparticles in solvents. J. Phys Chem. C, 115:18991-18998, 2011.

[46] K. Michael Salerno, Ahmed E. Ismail, J. Matthew D. Lane, and Gary S. Grest. Coating thickness and coverage effects on the forces between silica nanoparticles in water. J. Chem Phys., 140:194904-194911, 2014. 
[47] Ananth P. Kaushik and Paulette Clancy. Explicit all-atom modeling of realistically sized ligand-capped nanocrystals. J. Chem. Phys., 136:114702, 2012.

[48] Chetana Singh, Pradip K. Ghorai, Mark A. Horsch, Alicia M. Jackson, Ronald G. Larson, Francesco Stellacci, and Sharon C. Glotzer. Entropy-mediated patterning of surfactant-coated nanoparticles and surfaces. Phys. Rev. Lett., 99:226106, 2007.

[49] B. L. Peters, J. M. D. Lane, A. E. Ismail, and G. S. Grest. Fully atomistic simulations of the response of silica nanoparticle coatings to alkane solvents. Langmuir, 28:17443-17449, 2012.

[50] Brian J. Henz, Takumi Hawa, and Michael R. Zachariah. Mechano-chemical stability of gold nanoparticles coated with alkanethiolate sams. Langmuir, 24:773-783, 2008.

[51] Philipp Schapotschnikow, René Pool, and Thijs J. H. Vlugt. Molecular simulations of interacting nanocrystals. Nano Lett., 8:2930-2934, 2008.

[52] Kafui Tay and Fernando Bresme. Computer simulations of two dimensional gold nanoparticle arrays: the influence of core geometry. Mol. Sim., 31:515-526, 2005.

[53] J. Matthew D. Lane and Gary S. Grest. Assembly of responsive-shape coated nanoparticles at water surfaces. Nanoscale, 6:5132-5137, 2014.

[54] Stefania Rapino and Francesco Zerbetto. Dynamics of thiolate chains on a gold nanoparticle. Small, 3:386-388, 2007.

[55] Zhen Yang, Xiaoning Yang, Zhijun Xu, and Nannan Yang. Molecular simulations of structures and solvation free energies of passivated gold nanoparticles in supercritical $\mathrm{co}_{2}$. J. Chem. Phys., 133:094702, 2010.

[56] Ling Sun, Xiaoning Yang, Bin Wu, and Lunjiang Tang. Molecular simulation of interaction between passivated gold nanoparticles in supercritical $\mathrm{co}_{2}$. J. Chem Phys., 135:204703, 2011.

[57] Olga Lopez-Acevedo, Jaakko Akola, Robert L. Whetten, Henrik Grönbeck, and Hannu Häkkinen. Structure and bonding in the ubiquitous icosahedral metallic gold cluster Au144(SR)60. J. Phys Chem. C, 113:5035-5038, 2009.

[58] D. C. Rapaport. The Art of Molecular Dynamics Simulaions. Cambridge University Press, Cambridge, UK, 2004. 
[59] M. P. Allen and D. J. Tildesley. Computer Simulations of Liquids. Oxford University Press, New York, 1987.

[60] Tamar Schlick. Molecular Modeling and Simulation: An Interdisciplinary Guide. Springer, New York, 2010.

[61] M. E. Tuckerman. Statistical Mechanics: Theory and Molecular Simulation. Oxford University Press, Oxford, U.K., 2010.

[62] B. Smit and D. Frenkel. Understanding Molecular Simulations. From Algorithms to Applications. Academic Press, San Diego, 1996.

[63] Herman J. C. Berendsen. Simulating the Physical World. Cambridge University Press, Cambridge, UK, 2007.

[64] Andrew R. Leach. Molecular Modelling. Principles and Applications. Pearson Prentice Hall, New Jersey, USA, 2. auflage edition, 2001.

[65] J. M. Haile. Molecular Dynamics Simulation Elementary Methods. A WileyInterscience Publication, New York, 1992.

[66] Michael Griebel, Stephan Knapek, and Gerhard Zumbusch. Numerical Simulation in Molecular Dynamics. Springer, New York, 2007.

[67] Eric V. Anslyn and Dennis A. Dougherty. Modern Physical Organic Chemistry. University Science Books, California, 2006.

[68] Phillip M. Morse. Diatomic molecules according to the wave mechanics. ii. vibrational levels. Phys. Rev., 34:57-64, 1929.

[69] R. D. Blake. InInformation Biopolymers of Genes and Gene Expression. University Science Books, California, 2005.

[70] James E. Mark. Physical Properties of Polymers Handbook. Springer, Cincinnati, 2007.

[71] Nicolas Foloppe, Alexander D. MacKerell, and Jr. All-atom empirical force field for nucleic acids: I. parameter optimization based on small molecule and condensed phase macromolecular target data. J. Comp. Chem., 21:86-104, 2000. 
[72] E. K. Watkins and W. L. Jorgensen. Perfluoroalkanes: Conformational analysis and liquid-state properties from ab initio and monte carlo calculations. J. Phys. Chem. A, 105:4118-4125, 2001.

[73] A. Domfnguez, D. Frydel, and M. Oettel. Multipole expansion of the electrostatic interaction between charged colloids at interfaces. Phys. Rev. E, 77:020401-020405, 2008.

[74] Anatoliy Volkov and Philip Coppens. Calculation of electrostatic interaction energies in molecular dimers from atomic multipole moments obtained by different methods of electron density partitioning. J. Comp., 25:921-934, 2004.

[75] H. J. C. Berendsen, J. P. M. Postma, W. F. van Gunsteren, A. DiNola, and J. R. Haak. Molecular dynamics with coupling to an external bath. J. Chem. Phys., 81:3684-3690, 1984.

[76] Jerzy Leszczynski. Handbook of Computational Chemistry. Springer, Jackson, USA, 2012.

[77] Glenn J. Martyna. Constant pressure molecular dynamcis algorithm. J. Chem Phys., 101:4177-4189, 1994.

[78] William L. Jorgensen and Julian Tirado Rives. Potential energy functions for atomic-level simulations of water and organic and biomolecular systems. Proc. Nat. Acad. Sci., 102:6665-6670, 2005.

[79] Daniel J. Price and Charles L. Brooks. A modified tip3p water potential for simulation with ewald summation. J. Chem Phys., 121:10096-10103, 2004.

[80] William L. Jorgensen, Jayaraman Chandrasekhar, Jeffry D. Madura, Roger W. Impey, and Michael L. Klein. Comparison of simple potential functions for simulating liquid water. J. Chem Phys., 79:926-935, 1983.

[81] Michael W. Mahoney and William L. Jorgensen. A five-site model for liquid water and the reproduction of the density anomaly by rigid, nonpolarizable potential functions. J. Chem Phys., 112:8910-8922, 2000.

[82] Herbert Goldstein, Charles Poole, and John Safko. Classical Mechanics. AddisonWesley Publishing Company, New York, 2000. 
[83] Paul J. Flory. Principles of Polymer Chemistry. Cornell University, New York, 1953.

[84] V. Hornak, R. Abel, A. Okur, B. Strockbine, A. Roitberg, and C. Simmerling. Comparison of multiple amber force fields and development of improved protein backbone parameters. InterScience, 65:712-725, 2006.

[85] William L. Jorgensen, David S. Maxwell, and Julian Tirado-Rives. Development and testing of the opls all-atom force field on conformational energetics and properties of organic liquids. J. Am. Chem. Soc, 118:11225-11236, 1996.

[86] Michael Patra and Mikko Karttunen. Systematic comparison of force fields for microscopic simulations of nacl in aqueous solutions: Diffusion, free energy of hydration, and structural properties. J. Comp. Chem., 25:678-689, 2004.

[87] W. L. Jorgensen, J. Chandrasekhar, J. D. Madura, R. W. Impey, and M. L. Klein. Comparison of simple potential functions for simulating liquid water. J. Chem. Phys., 79:926, 1983.

[88] W. Humphrey, A. Dalke, and K. Schulten. Vmd: Visual molecular dynamics. J. Mol. Graph., 14:33-38, 1996.

[89] S. Plimpton. Fast parallel algorithms for short-range molecular dynamics. J. Comp. Phys., 117:1-19, 1995.

[90] J. P. Ryckaert, G. Ciccotti, and H. J. C. Berendsen. Numerical integration of the cartesian equations of motion of a system with constraints: Molecular dynamics of n-alkanes. J. Comput. Phys., 23:327-341, 1977.

[91] Henry Stephen, T Stephen, and Howard L Silcock. Solubilities of Inorganic and Organic Compounds. Pergamon Press, New York, 1963.

[92] Johan. Aaqvist. Ion-water interaction potentials derived from free energy perturbation simulations. J. Phys. Chem., 94:8021-8024, 1990.

[93] Nirmalya K. Chaki, Yuichi Negishi, Hironori Tsunoyama, Yukatsu Shichibu, and Tatsuya Tsukuda. Ubiquitous 8 and 29 kda gold:alkanethiolate cluster compounds: Mass-spectrometric determination of molecular formulas and structural implications. J. Am. Chem. Soc, 130:8608-8610, 2008. 
[94] Huifeng Qian and Rongchao Jin. Controlling nanoparticles with atomic precision: The case of $\mathrm{Au}_{144}\left(\mathrm{SCH}_{2} \mathrm{CH}_{2} \mathrm{Ph}\right)_{60}$. Nano Lett., 9:4083-4087, 2009.

[95] A. Christina, Fields-Zinna, Rajesh Sardar, Christopher A. Beasley, and Royce W. Murray. Electrospray ionization mass spectrometry of intrinsically cationized nanoparticles, $\left[\mathrm{Au}_{144 / 146}\left(\mathrm{SC}_{11} \mathrm{H}_{22} \mathrm{~N}\left(\mathrm{CH}_{2} \mathrm{CH}_{3}\right)^{3+}\right)_{\mathrm{x}}\left(\mathrm{S}_{(}\left(\mathrm{CH}_{2}\right)_{5} \mathrm{CH}_{3}\right)_{\mathrm{y}}\right]^{\mathrm{x}+}$. J. Am. Chem. Soc, 131:16266-16271, 2009.

[96] Huifeng Qian and Rongchao Jin. Ambient synthesis of $\mathrm{au}_{144}(\mathrm{sr})_{60}$ nanoclusters in methanol. Chem. Mater., 23:2209-2217, 2011.

[97] M. Heaven, A. Dass, K. Holt, P. White, and R. W. Murray. Crystal structure of the gold nanoparticle $\left.\left[\mathrm{N}\left(\mathrm{C}_{8} \mathrm{H}_{17}\right)_{4}\right]\left[\mathrm{Au}_{25} \mathrm{SCH}_{2} \mathrm{CH}_{2} \mathrm{Ph}\right)_{18}\right]$. J. Am. Chem. Soc, 130:3754$3755,2008$.

[98] Christine L. Heinecke, Thomas W. Ni, Sami Malola, Ville Mkinen, O. Andrea Wong, Hannu Hkkinen, and Christopher J. Ackerson. Structural and theoretical basis for ligand exchange on thiolate monolayer protected gold nanoclusters. J. Am. Chem. Soc, 134:13316-13322, 2012.

[99] F. Iori, R. Di Felice, E. Molinari, and S. Corni. Golp: An atomistic force-field to describe the interaction of proteins with $\mathrm{Au}(111)$ surfaces in water. J. Comp., 30:1465-1476, 2009.

[100] W. D. Cornell, P. Cieplak, C. I. Bayly, I. R. Gould, K. M. Merz, D. M. Ferguson, D. C. Spellmeyer, T. Fox, J. W. Caldwell, and P. A. Kollman. A second generation force field for the simulation of proteins, nucleic acids, and organic molecules. $J$. Am. Chem. Soc, 117:5179-5197, 1995.

[101] Jr. A. D. MacKerell, D. Bashford, M. Bellott, Jr. R. L. Dunbrack, J. D. Evanseck, M. J. Field, S. Fischer, J. Gao, H. Guo, S. Ha, D. Joseph-McCarthy, L. Kuchnir, K. Kuczera, F. T. K. Lau, C. Mattos, S. Michnick, T. Ngo, D. T. Nguyen, B. Prodhom, W. E. Reiher, B. Roux, M. Schlenkrich, J. C. Smith, R. Stote, J. Straub, M. Watanabe, J. Wiórkiewicz-Kuczera, D. Yin, and M. Karplus. All-atom empirical potential for molecular modeling and dynamics studies of proteins. J. Phys Chem. B, 102:3586-35616, 1998. 
[102] Marcus D Hanwell, Donald E Curtis, David C Lonie, Tim Vandermeersch, Eva Zurek, and Geoffrey R Hutchison. Avogadro: an advanced semantic chemical editor, visualization, and analysis platform. J. Cheminform., 4:2234, 2012.

[103] Amelie H. R. Koch, Gaetan Leveque, Sebastian Harms, Karmena Jaskiewicz, Max Bernhardt, Andreas Henkel, Carsten Sonnichsen, Katharina Landfester, and George Fytas. Surface asymmetry of coated spherical nanoparticles. Nano Lett., 14:4138, 2014.

[104] Hendrik Heinz, R. A. Vaia, B. L. Farmer, and R. R. Naik. Accurate simulation of surfaces and interfaces of face-centered cubic metals using 12-6 and 9-6 lennard-jones potentials. J. Phys Chem. C, 112:1728117290, 2008.

[105] Jure Gujt. Computer Simulations of Association of Simple Salts and Surfactants in Aqueous Solutions. PhD thesis, Faculty of chemistry and chemical technology, Ljubljana, 2015.

[106] Chris H. Rycroft. Voro++: A three-dimentional voronoi cell library in C++. Chaos, 19:041111, 2009.

[107] A. Sharake and J. A. Rupley. Environment and exposure to solvent of protein atoms. lysozyme and insulin. J. Mol. Biol., 79:351-364, 1973. 


\section{Appendices}

\section{A.1 Locations of files}

Some old data of different systems are in the directory

/imports/dd15/TheoDue/akantagi/

All the useful data of the simulations are in the the directory

faraday: /home/akantagi/

Data for the bulk solution systems, GNP systems with varied grafting density, and GNP systems with varied chain length and junction position are in three different directorytrees. The directory-tree for bulk solution systems is as follows:

AMBER-1.1-arithmetic AMBER-1.5-arithmetic AMBER-2.0-arithmetic OPLS-1.1-arithmetic OPLS-1.5-arithmetic OPLS-2.0-arithmetic CHARMM-1.1-arithmetic CHARMM-1.5-arithmetic CHARMM-2.0-arithmetic

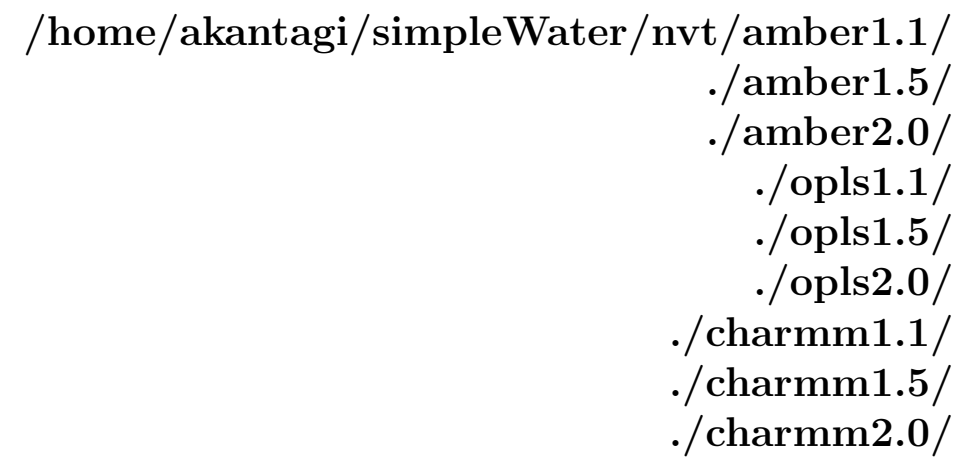

Above directories contain input files (laminputX.dat), restart files (restartX.dat) and trajectory files (trajectoryX.dcd). The files of the systems with geometric combination rules are stored in corresponding directories of the systems with arithmetic combination rules. For geometric combination, the file names are laminputgeoX.dat (input files), restartgeoX.dat (restart file) and trajectorygeoX.ded (trajectory files). Here, X indicates natural 
numbers like $1,2,3 \ldots$.

The directory-tree for GNPs with varied grafting density is as follows (all the directories are inside the parent directory /home/akantagi/group_goldnano/):

$\begin{array}{lr}\text { CH3-J0-CL11-T20 } & \text {./ch3ion_group/12long/20hairs/w20hair/data/ } \\ \text { CH3-J0-CL11-T40 } & \text {./ch3ion_group/12long/40hairs/w40hair/data/ } \\ \text { CH3-J0-CL11-T60 } & \text {./ch3ion_group/12long/60hairs/w60hair/data/ } \\ \text { COO-J0-CL11-T20 } & \text {./cooh_group/12long/20hairs/w20hair/data/ } \\ \text { COO-J0-CL11-T40 } & \text {./cooh_group/12long/40hairs/w40hair/data/ } \\ \text { COO-J0-CL11-T60 } & \text {./cooh_group/12long/60hairs/w60hair/data/ } \\ \text { NH3-J0-CL11-T20 } & \text {./nh4_group/12long/20hairs/w20hair/data/ } \\ \text { NH3-J0-CL11-T40 } & \text {./nh4_group/12long/40hairs/w40hair/data/ } \\ \text { NH3-J0-CL11-T60 } & \text {./nh4_group/12long/60hairs/w60hair/data/ }\end{array}$

Above directories contain input files (laminputchX.dat), restart files (restartchX.dat) and trajectory files (trajectorychX.dcd) of the simulations where AMBER-99 and CHARMM27 force field have been used.

The directory-tree for GNPs with varied chain length and junction position is as follows (all the directories are inside the parent directory /home/akantagi/LongChain/plot/):

\begin{tabular}{|c|c|}
\hline CH3-J0-CL5-T60 & $. / \mathrm{CH} 3 / 6 /$ data/ \\
\hline CH3-J0-CL11-T60 & $. / \mathrm{CH} 3 / 12 /$ data/ \\
\hline CH3-J4-CL11-T60 & $. / \mathrm{CH} 3 / 12 \mathrm{y} 4 /$ data/ \\
\hline CH3-J8-CL11-T60 & ./CH3/12y8/data/ \\
\hline CH3-J0-CL17-T60 & $. / \mathrm{CH} 3 / 18 /$ data/ \\
\hline CH3-J4-CL17-T60 & ./CH3/18y4/data/ \\
\hline CH3-J8-CL17-T60 & $. / \mathrm{CH} 3 / 18 y 8 /$ data/ \\
\hline CH3-J12-CL17-T60 & ./CH3/18y12/data/ \\
\hline CH3-J12-CL17-T70 & ./CH3/18y8eq/data/ \\
\hline CH3-J12-CL17-T80 & ./CH3/18y12eq/data/ \\
\hline CH3-J0-CL23-T60 & $. / \mathrm{CH} 3 / 24 /$ data/ \\
\hline
\end{tabular}




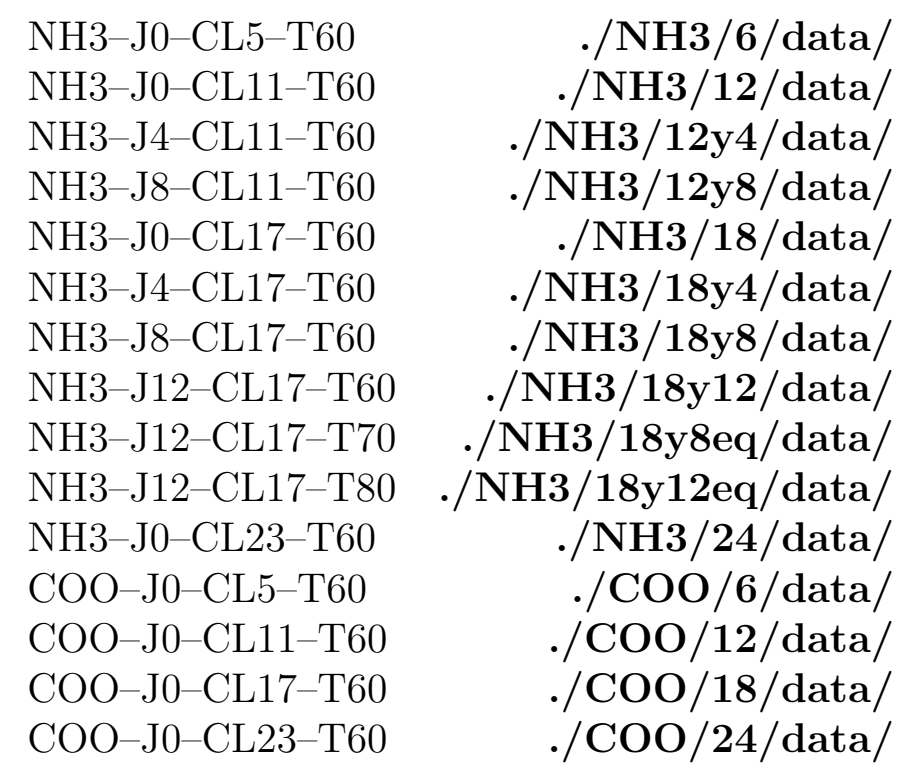

Above directories contain input files (laminputX.dat), restart files (restartX.dat) and trajectory files (trajectoryX.dcd) of the simulations where OPLS-AA force field have been used. 


\section{A.2 Color maps of surface access of $\mathrm{NH}_{3}^{+}$terminated systems with different grafting densities}
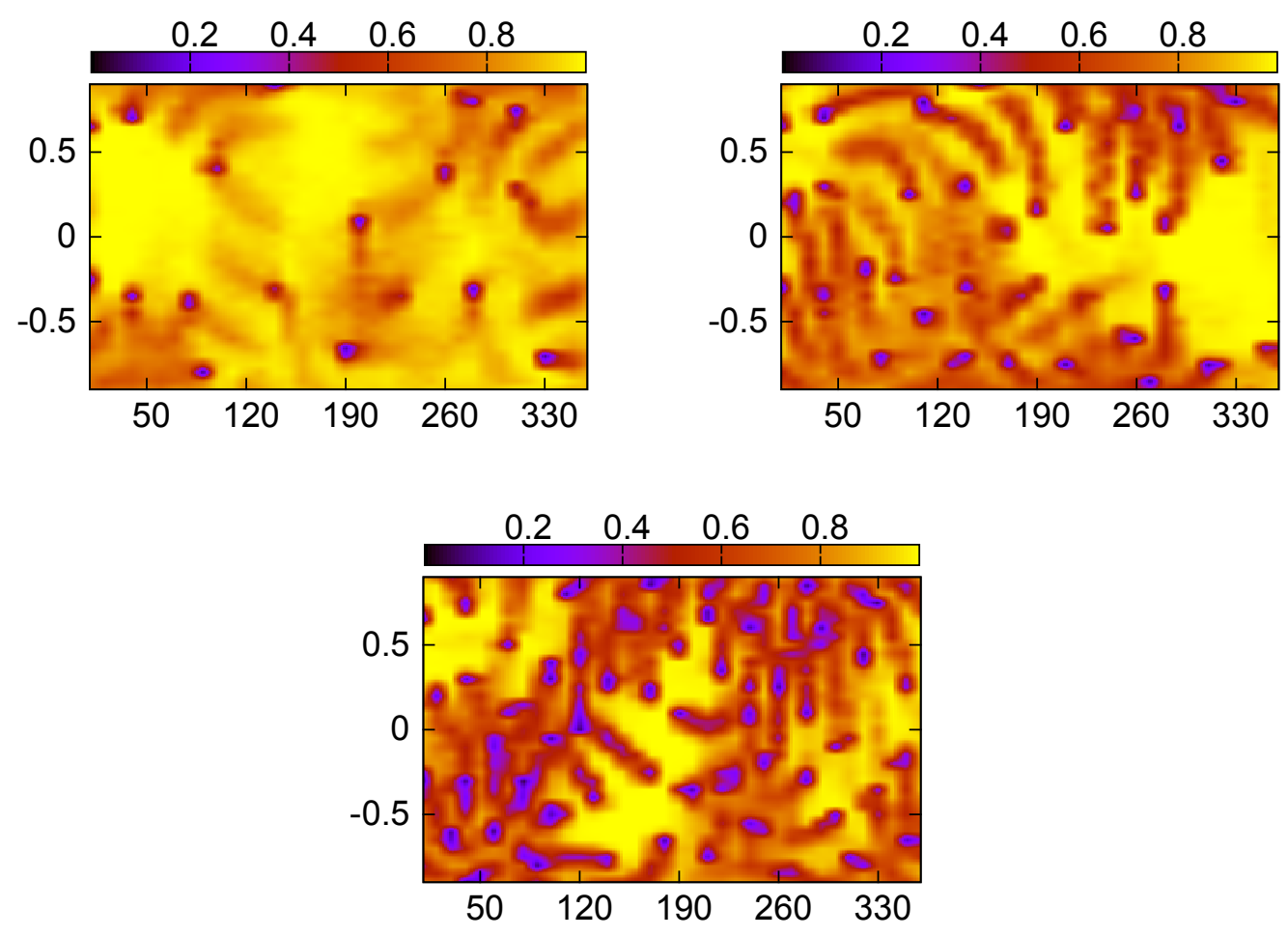

Figure A.1: Color maps of surface access. Value 1 (yellow) indicates that a water molecule is closest to the GNP surface and a value 0 indicates a chain carbon atom is closest to the surface. All maps show the entire surface of the unit sphere (in the laboratory frame) with the cosine of the polar angle $\theta$ between -1 and 1 changes along vertical axis and the azimuthal angle $\phi$ between 0 and 360 changes along horizontal axis. $\mathrm{NH}_{3}^{+}$terminated chains with $d_{f}=1 / 3$ (top left), $2 / 3$ (top right) and 1 (bottom). 


\section{A.3 Time averaged angle distribution}
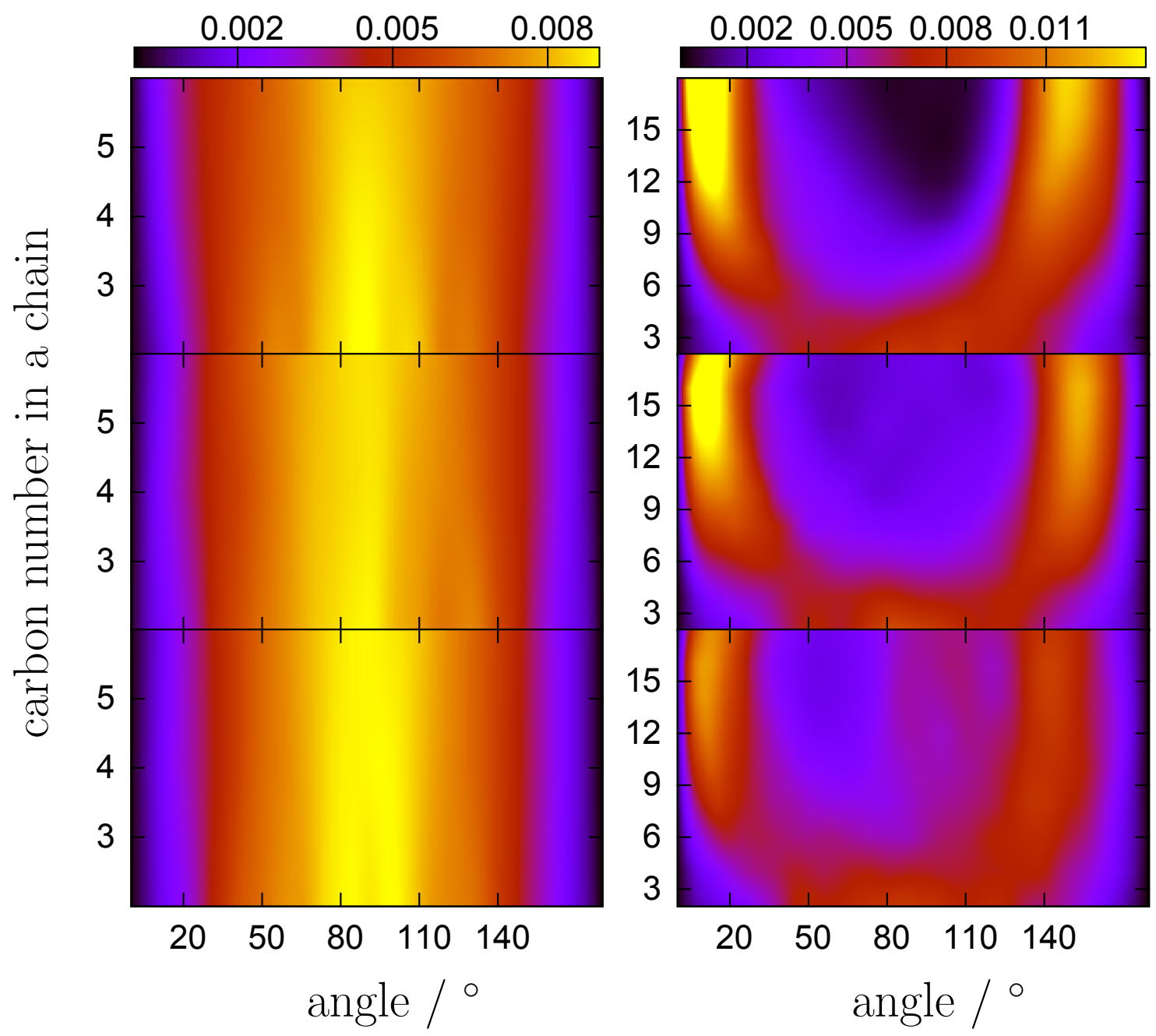

Figure A.2: Time averaged angle distribution of $\mathrm{CH}_{3}, \mathrm{COO}^{-}$and $\mathrm{NH}_{3}^{+}$groups terminated systems (Top to bottom). Left and right columns are for the GNPs systems with $C_{l}=5$ and 17. 


\section{A.4 Color maps of surface access with different chain length}
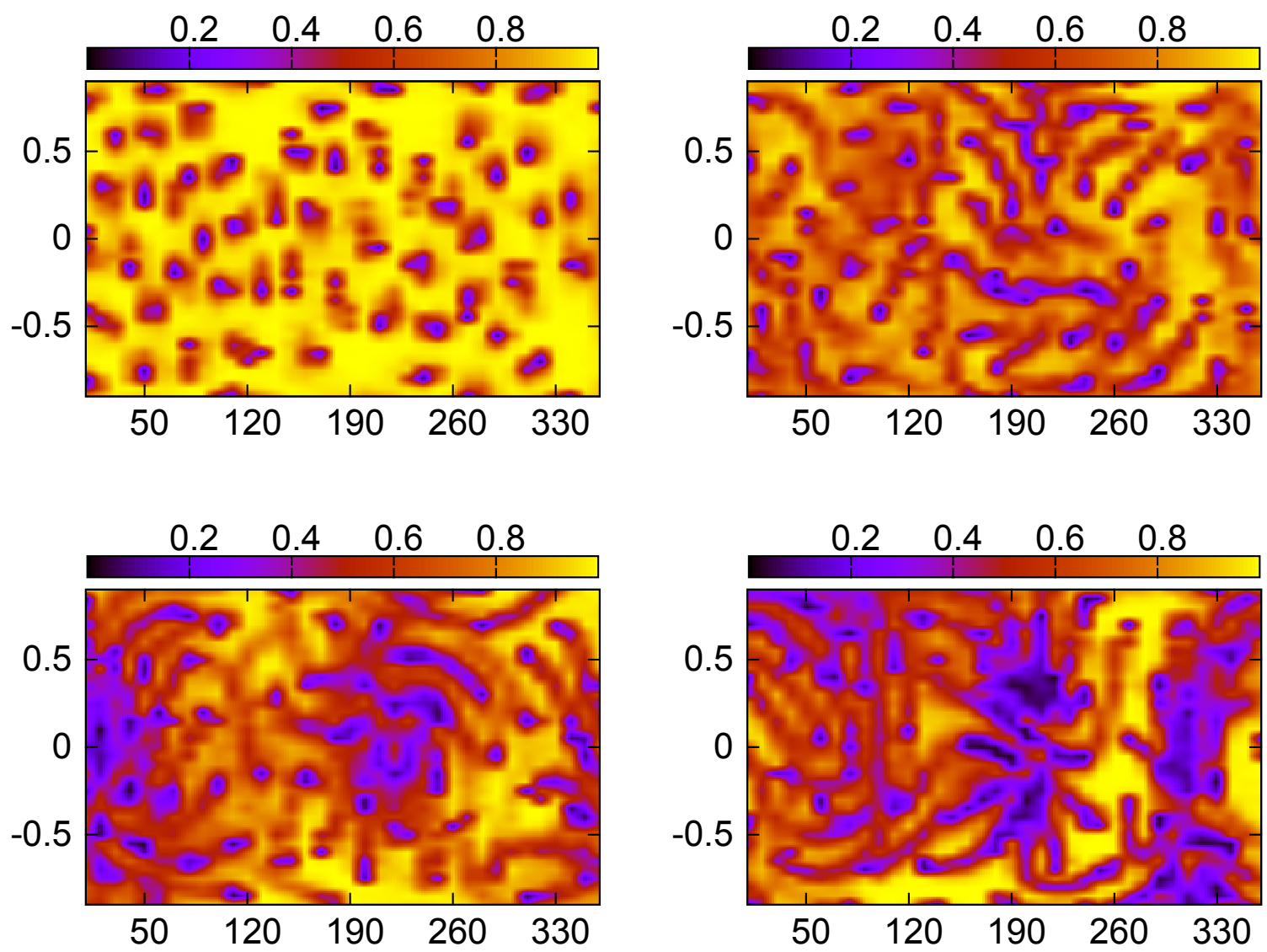

Figure A.3: Color maps of surface access. Value 1 (yellow) indicates that a water molecule is closest to the GNP surface and a value 0 indicates a chain carbon atom is closest to the surface. $\mathrm{NH}_{3}^{+}$terminated chains with chain length 5 (top left), 11 (top right), 17 (bottom left), 23 (bottom right). All maps show the entire surface of the unit sphere (in the laboratory frame) with the cosine of the polar angle $\theta$ between -1 and 1 changes along vertical axis and the azimuthal angle $\phi$ between 0 and 360 changes along horizontal axis. 


\section{A.5 Color maps of surface access of branched chain systems of chain length 11}
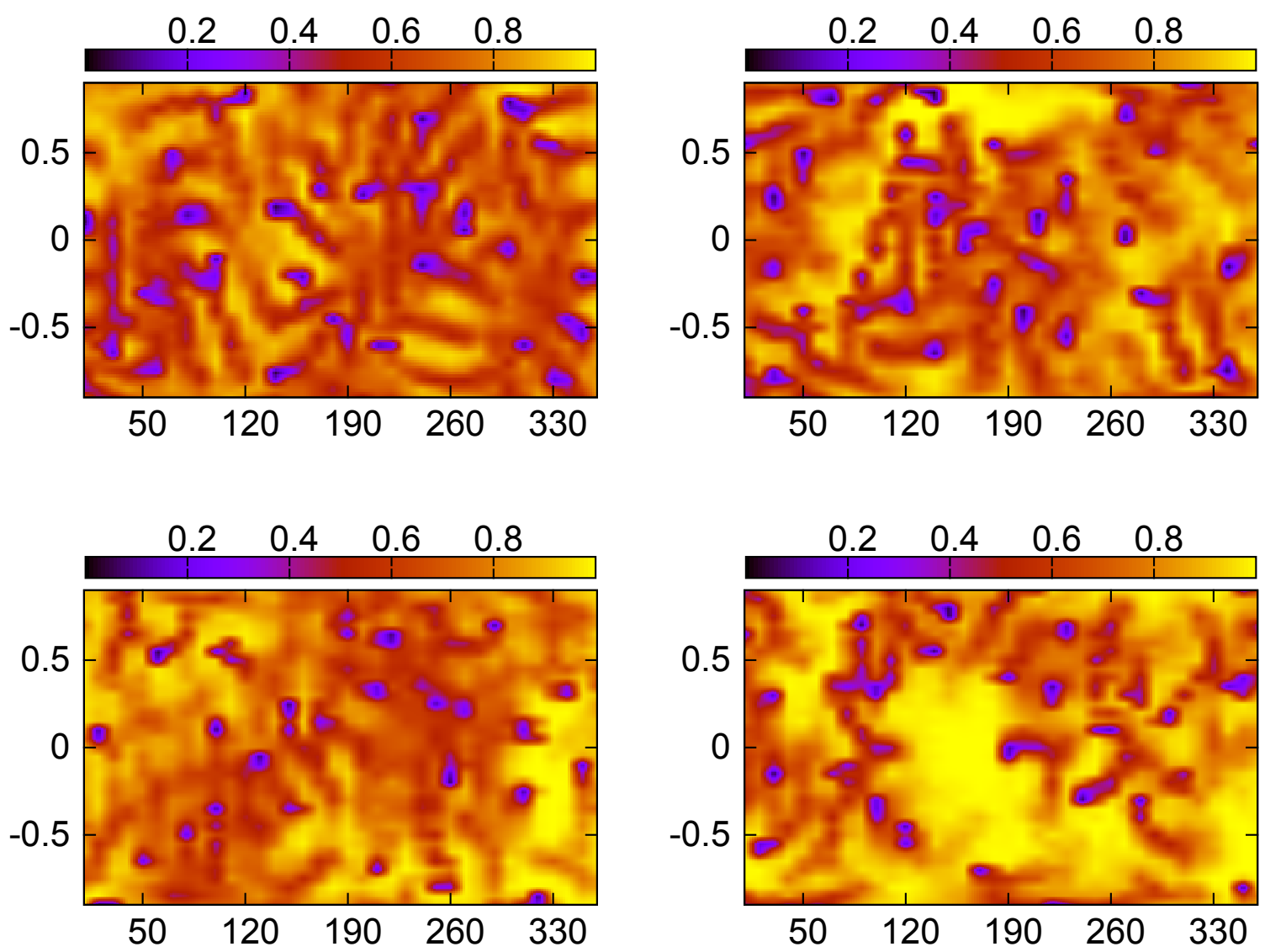

Figure A.4: Color maps of surface access of GNPs with $C_{l}=11$. Value 1 (yellow) indicates that a water molecule is closest to the GNP surface and a value 0 indicates a chain carbon atom is closest to the surface. Left column: $\mathrm{CH}_{3}$ group terminated branched chains of junction at 4th and 8th carbon atom (from to bottom). Right column: $\mathrm{NH}_{3}^{+}$ group terminated branched chains of junction at 4th and 8th carbon atom (from top to bottom). All maps show the entire surface of the unit sphere (in the laboratory frame) with the cosine of the polar angle $\theta$ between -1 and 1 changes along vertical axis and the azimuthal angle $\phi$ between 0 and 360 changes along horizontal axis. 
A.6. Radius of gyration and time averaged chain length of $\mathrm{NH}_{3}^{+}$group terminated chains

\section{A.6 Radius of gyration and time averaged chain length of $\mathrm{NH}_{3}^{+}$group terminated chains}

Table A.1: Radius of gyration and chain length

\begin{tabular}{|l|l|l|l|l|}
\hline Systems & $\mathrm{R}_{g}(\AA)$ & $\begin{array}{l}\mathrm{R}_{g} \text { Standard } \\
\text { deviation }\end{array}$ & Chain length $(\AA)$ & $\begin{array}{l}\text { Chain length } \\
\text { Standard de- } \\
\text { viation }\end{array}$ \\
\hline NH3-J0-CL12-T60 & 16.11 & 0.11 & 12.91 & 0.09 \\
\hline NH3-J4-CL12-T60 & 15.16 & 0.09 & 11.57 & 0.10 \\
\hline NH3-J8-CL12-T60 & 15.14 & 0.25 & 11.27 & 0.13 \\
\hline NH3-J0-CL18-T60 & 18.80 & 0.09 & 19.55 & 0.21 \\
\hline NH3-J4-CL18-T60 & 16.93 & 0.12 & 16.70 & 0.20 \\
\hline NH3-J8-CL18-T60 & 16.45 & 0.17 & 16.09 & 0.27 \\
\hline NH3-J12-CL18-T60 & 16.55 & 0.32 & 15.94 & 0.26 \\
\hline
\end{tabular}


Der Lebenslauf ist in der Online-Version aus Gründen des Datenschutzes nicht enthalten. 



\section{Declaration}

I hereby declare that this $\mathrm{PhD}$ thesis entitled "Conformational Equilibria of Organic Adsorbates on Nanostructures in Aqueous Solution. MD Simulations" has been carried out by me under the supervision of Prof. Eckhard Spohr at University of Duisburg-Essen. This thesis is the result of my own independent research work. I have produced this thesis without any assistance from third parties, except where otherwise stated. This thesis or part of this thesis has not been presented before to any examination board.

Essen, April 2016

(signature) 